\title{
Evaluation of Background Mercury Concentrations in the SRS Groundwater System (U)
}

December 7, 1998

Brian B. Looney

Dennis G. Jackson

Miles E. Denham

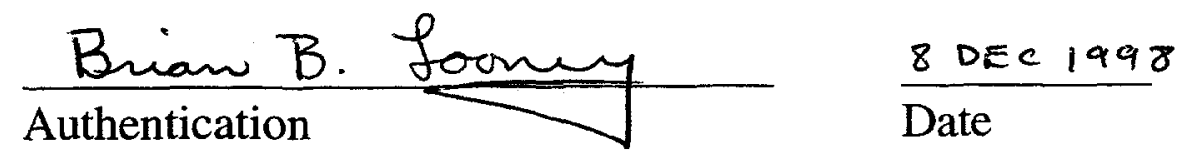

Westinghouse Savannah River Company

Savannah River Site

Aiken, South Carolina 29808 


\section{DISCLAIMER}

This report was prepared as an account of work sponsored by an agency of the United States Government. Neither the United States Government nor any agency thereof, nor any of their employees, make any warranty, express or implied, or assumes any legal liability or responsibility for the accuracy, completeness, or usefulness of any information, apparatus, product, or process disclosed, or represents that its use would not infringe privately owned rights. Reference herein to any specific commercial product, process, or service by trade name, trademark, manufacturer, or otherwise does not necessarily constitute or imply its endorsement, recommendation, or favoring by the United States Government or any agency thereof. The views and opinions of authors expressed herein do not necessarily state or reflect those of the United States Government or any agency thereof. 


\section{DISCLAIMER}

Portions of this document may be illegible in electronic image products. Images are produced from the best available original document. 


\section{Executive Summary}

Mercury analyses associated with the A-01 Outfall have highlighted the importance of developing an understanding of mercury in the Savannah River Site groundwater system and associated surface water streams. This activity is critical based upon the fact that the EPA Ambient Water Quality Criteria (AWQC) for this constituent is $0.012 \mu \mathrm{g} / \mathrm{L}$, a level that is well below conventional detection limits of 0.1 to $0.2 \mu \mathrm{g} / \mathrm{L}$. A first step in this process is obtained by utilizing the existing investment in groundwater mercury concentrations (20,242 records) maintained in the SRS geographical information management system (GIMS) database. Careful use of these data provides a technically defensible initial estimate for total recoverable mercury in background and contaminated SRS wells.

Methods of order statistics have been combined with probability plotting and partitioning techniques to estimate the background groundwater concentration as $0.093 \mu \mathrm{g} / \mathrm{L}$. The data set for this estimate included 518 values with estimated concentrations, 856 values that were reported below a nominal detection limit, and 350 values with actual reported concentrations. The analysis process included the partitioning of wells into two distinct populations, one associated with ambient, background mercury concentrations, and the other associated with groundwater that has possibly been impacted by SRS activities.

The analysis provides a reasonable upper bound on the estimated ambient, background concentration for mercury in the SRS groundwater system. Uncertainties associated with the analysis include possibility of elevated concentrations in wells due to grout contamination; the potential for increased concentrations resulting from laboratory contamination, and the use of data only from a relatively brief sampling window. In applying the estimate to the identification of reasonable surface water quality, uncertainties also exist. These uncertainties relate to interactions in the hyporheic zone and the species of mercury present in the stream. These uncertainties would be best managed by a limited amount of speciated analysis of both groundwater and surface water samples. Since mercury can exist in several forms in the environment, definitive data with appropriate detection limits is needed to support decisions regarding stream quality and site discharge criteria.

The methods discussed and utilized are not limited exclusively to mercury analysis. These robust techniques are valid for analysis and partitioning of large data sets that are postulated to comprise a combination of normal or log normal populations. Through the application process the hypothesis is validated in a simple graphical manner and quantitative results are obtained. The analysis illustrates one possible technique of incorporating both data that is traditionally unused and data that is considered unusable with validated data. Once performed the results using this combination and analysis process can be used to support environmental analysis and effective decision making. 


\section{Table of Contents}

Executive Summary

iii

Table of Contents

iv

List of Figures

$\mathbf{v}$

List of Tables

Objective

Background

Methods

Developing Monitoring Well Data Set

Developing the Best Estimate of Mercury Concentration for each Well

Probability Plots

Results and Analysis

Recommendations and Conclusions

References

Appendix A: Estimated Median Concentrations for SRS Groundwater Wells Associated with Background Population.

Appendix B: Estimated Median Concentrations for SRS Groundwater Wells for Undetermined Population29

Appendix C: Estimated Median Concentrations for SRS Groundwater Wells Associated with the Impacted Population.

Appendix D: SRS Groundwater Wells with all Reported Values Below Nominal Detection Limit. 


\section{List of Figures}

Figure 1: Cumulative Histogram of Mercury Concentrations from 19,917 Groundwater Measurements at the Savannah River Site including Estimated and Non-Detect values.

Figure 2: Flowchart Outlining Method Used to Obtain Best Estimate of Concentration Considering "NonDetects and "Estimated" Values.

Figure 3: Application of Probability Plots in Identifying and Partitioning of two Log-Normal Populations from Combined Data Sets. (Adopted from Sinclair, 1976).

Figure 4: Log Probability Plot of Mercury Concentration from 1,724 Wells at the Savannah River Site. Populations partitioned into an Impacted Population and a Background Population.

Figure 5: Map of the Savannah River Site Showing the Location and Distribution of Wells that are in the Background Population, in the Impacted Population, and whose population is Undetermined.

\section{List of Tables}

Table 1 Summary Information from Partitioning Analysis of Groundwater Mercury Concentration from 1,724 Monitoring Wells at the Savannah River Site. 


\section{Objective}

The overall objective of this investigation was to develop a technically based estimate of background concentrations for mercury (total recoverable mercury) for groundwater underlying the Savannah River Site (SRS). The selected methods were developed by exploration geochemists and maximize the usefulness of the large amount of high quality data that exist in the SRS geochemical database. Further, the approach provides initial estimates of background and impacted groundwater concentrations and information to support scientists, engineers and managers in developing environmental protection and remediation strategies at SRS.

\section{Background}

The Savannah River Site was constructed during the early 1950 s to produce the basic materials used in the fabrication of nuclear weapons, primarily tritium and plutonium-239. To accomplish this mission five nuclear reactors were built on the site and were used to produce nuclear materials by irradiating target materials with neutrons. In addition to the direct production facilities, numerous support facilities were also constructed. This included two chemical separation plants, a heavy water extraction plant, a nuclear fuel and target fabrication facility, waste management facilities, research and support laboratories, and administrative facilities.

Although not a product of these processes, mercury was used in several aspects of facility operations. Processes and systems that incorporated mercury included the Separation Areas where mercury was used as a process aid, the Tritium Facilities where mercury was used in the gas handling systems, and in many of the numerous experimental, laboratory, and miscellaneous support facilities. With the exception of the mercury that was released as a vapor directly to the atmosphere, spent mercury was directed to one of several Site waste facilities. These facilities included underground waste tanks, the Solid Waste Disposal Facility, the Sanitary Landfill, and other miscellaneous waste facilities at the Savannah River Site. These miscellaneous waste facilities included seepage and overflow basins and retention ponds. Details and inventory estimates associated with these and other SRS facilities have been recently documented (Kvartek et.al. 1994). Interested readers should refer to this work for a more comprehensive discussion of mercury in the Savannah River Site environment. This reference document serves as a thorough reference regarding specific aspects of mercury usage and disposal throughout the Site history.

In addition to the mercury that was directly used and released as part of process operations, mercury is also present both naturally and from sources not indigenous to the Savannah River Site. These sources include mercury present in water drawn from the Savannah River, mercury that is present in precipitation, and trace amounts of mercury that are present in subsurface soils. The presence of elevated mercury in the Savannah River is a result of historical releases near the city of Augusta, Georgia. These releases have resulted in the presence of mercury in Savannah River sediments and water upstream of the Savannah River Site. During process operations, river water, containing mercury, was pumped and used as non-contact cooling water in the Site's reactors (Kvartek et.al. 1994). Following usage, this water was released on-site to surface streams where the mercury collected in stream sediments. As a result mercury is now commonly found in surface waters and fish in some areas of the Savannah River Site. Investigations have compared the accumulation of mercury from fish collected from Site surface waters and from upstream regions of the Savannah River (Newman and Messier, 1994). The results of this investigation indicated that no significant differences in mercury concentrations exist with 
samples collected on-site versus those collected off-site. The investigation did indicate that a significant difference exists between samples associated with the Savannah River versus samples that were isolated from the river.

Definitive data regarding mercury contributions from precipitation is sparse in both the spatial and temporal perspective. The only identified resource for this data is from the Mercury Deposition Network (Lindberg and Vermette, 1995;Vermette et.al. 1995; and Welker and Vermette, 1996). The Mercury Deposition Network (MDN) is a network of nationwide monitoring and reporting stations with the objective of developing a national database of weekly concentrations of total mercury in precipitation and the seasonal and annual flux of total mercury in wet deposition. This system is part of the National Atmospheric Deposition Program/National Trends Network (NADP/NTN) and is a nationwide network of precipitation monitoring sites. The network is a cooperative effort between many different groups, including the State Agricultural Experiment Stations, U.S. Geological Survey, U.S. Department of Agriculture, and numerous other governmental and private entities. Data collected from this program will be used to develop information on spatial and seasonal trends in mercury deposited to surface waters, forested watersheds, and other sensitive receptors. A monitoring station for this network is located in Congaree Swamp National Monument, south of Columbia, South Carolina. Data collected since 1995 indicates that the mercury concentration in precipitation is approximately $0.010 \mu \mathrm{g} / \mathrm{L}$.

Although not a common element, mercury is naturally present in soils beneath the Savannah River Site above detectable limits. An estimate on the amount of mercury present in the subsurface can be obtained from the SRS Background Soil Study that was concluded in 1990 (Looney et.al. 1990). The objective of this program was to characterize the geochemical and physical properties of the soils and shallow sediments at the Savannah River Site. Mercury data collected in conjunction with this investigation reported that approximately $80 \%$ of the 168 samples were below the method detection limits. The reported data ranged from $<0.01 \mu \mathrm{g} / \mathrm{g}$ of soil to $0.89 \mu \mathrm{g} / \mathrm{g}$ of soil with a median below $0.1 \mu \mathrm{g} / \mathrm{g}$ of soil. None of the 24 detected values were below the minimal detection limit. The study reported that the concentrations did not exhibit a clear pattern in terms of soil series or layer.

Within groundwater mercury can take many forms, some of these forms are more mobile and others sorb strongly onto soils. Mercury that is present in the soils will usually exist in a stable form, leaching slowly to the groundwater due to weathering processes. The mobility and speciation of mercury in aqueous solution is complex and dependent upon a variety of specific parameters. Under most natural environmental conditions, mercury tends to sorb strongly on the soil, associating with iron oxides and hydroxides, organic coatings, or exchange sites on the surfaces of the minerals. Thus, soils that contain clays and/or organic and oxide coatings tend to maximize sorption. Investigations have shown that chloride complexes, favored by low $\mathrm{pH}$ may significantly increase the mobility of mercury even at relatively low chloride ion concentrations. These forms of mercury (e.g. chloride complexes such as $\mathrm{HgCl}_{2}{ }^{0}$ ) tend to migrate more rapidly than more common forms of mercury (e.g. $\mathrm{Hg}^{+}$and $\mathrm{Hg}(\mathrm{OH})_{2}{ }^{0}$ ) which are typically more strongly attenuated (Anderson, 1970). Limited investigations have indicted that these processes may occur at the Savannah River Site and that mercury may not be significantly adsorbed in certain soil areas (Looney, 1994 and Denham and Strom 1994).

The transport and migration of mercury that is present in the groundwater in a mobile form will be governed by advective transport process. From a conceptual perspective these processes are 
directed by the hydrologic cycle and includes precipitation deposition in groundwater recharge areas, leaching from soils based upon local geochemical interactions, followed by discharge to surface streams and bodies. The complex interaction between groundwater and surface water have been recently presented (USGS, 1998). Although not directed at mercury, these discussions provide valuable insight on the relationship and impact between groundwater and surface water resources, and the role of this "hyporheic zone" in controlling impacts from groundwater plumes. These relationships and associated effects are enhanced when groundwater resources are discharged directly to surface streams and may have significant effects on stream quality.

Emerging events at the A-01 and other site outfalls have highlighted the importance of developing an understanding of mercury in the Site groundwater and surface streams. Outfalls serve as regulatory controlled release points for water used in site process activities. The majority of the water released at these points originates in some form from groundwater resources beneath the Site. In certain areas outfall releases are associated with active groundwater corrective action programs, e.g. the A/M Area. Releases in these areas further emphasize the need for expanded understanding of mercury in the Site groundwater and surface streams. This added awareness is directed by the strict Ambient Water Quality Criteria (AWQC) for mercury. These criteria govern and impact outfall permit levels. The AWQC level for mercury is $0.012 \mu \mathrm{g} / \mathrm{L}$, a level that is well below conventional (i.e. EPA SW-846) detection limits and EPA primary drinking water standards. The strict limit is based on toxicology studies with the assumption that all of the mercury in the stream is methyl mercury, the most toxic form, with a high bio-accumulation factor. Even though naturally occurring mercury may be present above AWQC criteria, conventional SW-846 analysis prevents identification due to method detection limits. This would imply an ambient, un-impacted stream concentration above the AWQC criteria of $0.012 \mu \mathrm{g} / \mathrm{L}$ but below SW-846 detection limits (typically $0.2 \mu \mathrm{g} / \mathrm{L}$ ). Limitations in method detection limits, combined with outfall averaging procedures often result in exceedance of permit limits. Until definitive ambient stream data is obtained with suitable detection limits below AWQC criteria, ambient background stream concentrations cannot be ascertained. Although historically not available, a reasonable bound on the background stream concentrations can be obtained based upon background concentrations of mercury in the groundwater. This estimate will provide the following information:

- Assist in establishing reasonable permit levels for groundwater discharges to surface steams,

- Aid in identifying regions which may have been impacted by mercury usage,

- Provide a bound for mercury concentrations in site surface streams, and

- Illustrate techniques suitable for partitioning and establishing background concentrations.

\section{Methods}

Identifying a technically based estimate value for the background concentrations of mercury in the groundwater for the Savannah River Site involved several carefully managed activities. In order to prevent biasing of the results, during each of the described activities extreme care and caution was taken so that each step was rational and systematic. As discussed in subsequent sections, this systematic approach was important in selecting and establishing the "average" or "Best Estimate" of mercury concentration for individual monitoring wells. Once these individual "Best Estimated" concentrations were determined, these data were used with order statistics and 
analyzed using probability plots. This allowed for the identification and partitioning of the data into two populations, one population that is considered typical of natural (background) conditions, and the second population that is considered to represent those wells, i.e. groundwater, that have been impacted by SRS operations.

\section{Developing Monitoring Well Data Set}

The initial process of identifying background mercury levels involved the collection, screening, and assembly of historical monitoring well data available for the Site's monitoring well network. Sampling and analysis of groundwater is an important aspect of the environmental protection, awareness, and remediation program at the Savannah River Site. Analytical results from monitoring wells are maintained and used to provide data necessary to identify and monitor groundwater conditions and contaminants, assist with environmental compliance and DOE directives, and to support basic and applied research. Combined, systematic analysis of historical data from active and abandoned wells allows investigators to establish a background concentration for a particular constituent and to identify regions that may be potentially impacted.

Historical groundwater data at the Savannah River Site is maintained as an integral part of the SRS Groundwater Monitoring Program. This program evaluates all data systematically to provide high-quality information for reporting on the environmental monitoring and cleanup efforts at SRS. Data verification and validation are ongoing, interactive processes, usually completed within 60 days after the last data are received from a quarterly sampling event. Once analytical data is received from the contractor laboratory, the data is incorporated into the Geochemical Information Management System (GIMS). GIMS is a combination of hardware, software, data, and procedures that supports environmental data management activities. The GIMS Data Review Module provides automated data loading, validation and verification functions, data editing, management of data review status, report generation, and data review QA. Data editing is allowed in order to correct errors in loaded analytical, field, and shipping data. When the review process is complete, data are loaded into permanent production database tables in GIMS and are available sitewide (GIMS, 1997).

Prior to loading into GIMS, the analytical data from the laboratories are reviewed for errors and unusual results before releasing the data to the production databases. The laboratories review and comment on suspect data. Errors typically identified during data loading include incorrect sample dates, run dates, and sample identifications; incorrectly entered analytical units, methods, and corresponding detection limits; and incorrect dilution factor calculations. Analytical results that appear different from historical data collected since 1991 are brought to the attention of the appropriate laboratory. Thus, the laboratory is able to identify problems with some of the analyses, including incorrect dilution factor calculations and data entry errors. To determine if a new analytical result for a sampling site is similar to or relatively higher or lower than historical results, new results for each well are compared to historical results using systematic procedures.

In addition to these QA/QC checks, site representatives review the analytical narratives received from the contract laboratories. These narratives are used as reference materials throughout the data validation process. The narratives include discussions on the following problems: QA samples that do not meet the criteria specified by the analytical method, problems with matrix interference, sample-specific adjustments to the method caused by high concentrations of some constituents, problems with sample preservation and holding time, instrument calibration 
problems, and contaminated blanks. The narratives also include additional information about COC and analytical data. Quarterly reports (e.g. WSRC, 1997a through 1997d) are published and archived documenting this rigorous $Q A / Q C$ process such that end-users are assured that the data available in the production databases meets the highest data quality standards and objectives.

To support the estimation of background mercury concentrations in groundwater, historical data for "Mercury, Total Recoverable" was collected for all the wells in GIMS using ORACLE database query tools. This query returned a data set of 20,242 records containing historical mercury concentrations for active and abandoned monitoring wells, both on and off the Savannah River Site, as well as laboratory blank samples. The period of record for this data ranged from October 1992 through the most recent groundwater sampling event that is on record, August 1998. This data set was reviewed and critiqued using the following criteria:

- Removal of "Lab Blank" samples (275 records),

- Removal of Data Flagged " $R$ " indicating poor performance requirements in the sample or associated quality control issues (20 Records).

- Removal of Data Flagged " $V$ " indicating that the constituent was detected in both the sample and the associated method blank (30 Records).

As a result of these additional QA/QC processes the bulk data set contained 19,917 historical values for mercury distributed at monitoring wells around the Savannah River Site. The cumulative frequency histogram for this data is presented in Figure 1. Within Figure 1 the data are identified by the Result Qualifier Code that is associated with each result. As shown in this figure a large portion of this data set is flagged as "Non-Detect." In the GIMS system "NonDetects" are designated by a result qualifier code of " $U$ " indicating that the material was analyzed for but not detected. In these samples the result concentration is less than the samplespecific estimated quantitation limit. In the bulk data set approximately $78 \%(15,557)$ of the values were reported as "Non-Detect." The most prominent "Non-Detect" values are indicated by the vertical lines on the cumulative frequency histogram at total recoverable mercury concentrations of $0.1 \mu \mathrm{g} / \mathrm{L}, 0.2 \mu \mathrm{g} / \mathrm{L}, 0.67 \mu \mathrm{g} / \mathrm{L}$, and $1.0 \mu \mathrm{g} / \mathrm{L}$. In addition to these "Non-Detect" values, the bulk data set contained approximately $9 \%(1,721)$ of the records which had a " $\mathrm{J}$ " as the Result Qualifier Code, indicating that the analytical result is an estimated quantity. The estimated data ranged from $0.015 \mu \mathrm{g} / \mathrm{L}$ to $18.2 \mu \mathrm{g} / \mathrm{L}$. As shown in the cumulative frequency histogram the majority of these values are below $0.2 \mu \mathrm{g} / \mathrm{L}$. As indicated in the histogram these "Estimated" values provide a great deal of definition for the lower range of the data set. The remaining $13 \%(2,639)$ of the bulk data set contained quantifiable mercury levels that were reported. These data ranged from $0.067 \mu \mathrm{g} / \mathrm{L}$ to $4,300 \mu \mathrm{g} / \mathrm{L}$.

\section{Developing the Best Estimate of Mercury Concentration for each Well}

Review of the total mercury concentration values in the groundwater database suggests that a preliminary estimate of a background concentration (below the detection limit) is possible by incorporating the values flagged with a " $\mathrm{J}$ ". " $\mathrm{J}$ " designates that the value is an estimate. For many wells, the laboratory analyst provides an estimate of below nominal detection limit concentration in one or more analysis periods. Use of these data to develop a preliminary estimate of background is consistent with EPA (1992) guidance for data usability in risk 
assessment: "With proper interpretation, the results of analyses which are flagged " $\mathrm{J}$ " can often be used in making decisions."

These "Estimated" values are used and incorporated to determine the best possible estimate of background mercury concentration for SRS groundwater. To support this goal, a median value of the data (or of the data estimates) was chosen as the most representative value for each well. This approach has two positive impacts. First, it assigns each well a concentration that is representative of a single location (an assumption that is consistent with the subsequent geostatistical technique that is implemented). Second, the median, "best value," approach minimizes the potential for bias associated with the simpler approach of using "every analysis" in the GIMS production database. Several types of bias would tend to result from the "every analysis" evaluation approach:

- Some wells have many more analyses than others and these would be given undue weight in determining background levels (in fact, it is likely that contaminated wells would typically have the most analyses further biasing any estimates), and

- The data for many wells cluster around a consistent low value with only a single very high result. Use of a median value minimized the impact of such data and results in a more conservative (low) and realistic estimate of background concentration.

Because of the complexity of the low concentration data and the variety of possible situations in the large data set, a consistent approach was developed to estimate the median value. Each well with data reported above the nominal detection limit, at the nominal detection limit, or with estimated values below the nominal detection limit was included in the evaluation and was individually evaluated. The resulting "best estimate" for the well was maintained for subsequent analysis using geo-statistics. The flowchart presented in Figure 2 summarizes the steps (rules) used for generating the estimates for an individual well. Examples for each case are provided in italics.

Using this approach, the entire data set was evaluated with the following results for the 1,724 estimated median well concentrations that were generated:

- 518 wells had specified estimates of median concentrations at concentrations below the nominal detection limit $(0.2 \mu \mathrm{g} / \mathrm{L})$

- 856 wells had estimated median concentrations reported as the nominal detection limit $(<0.2$ $\mu \mathrm{g} / \mathrm{L})$

- 350 wells had specified estimates of median concentrations at concentrations above $0.2 \mu \mathrm{g} / \mathrm{L}$

\section{Probability Plots}

With a composite data set developed that represents the best estimated concentration at each position, geo-statistical methods were applied to partition the data set and estimate statistical parameters associated with each population. Sinclair (1976) documents the use of probability graphs and geo-statistical methods in mineral exploration. In applications regarding the location of mineral resources, a primary objective is to determine where target elements are elevated in concentration and where concentrations are not elevated (background). As shown on Figure 3, this process is relatively straightforward using probability graphs. In fact, the graphs provide quantitative estimates of the statistical properties of the distributions and standard-defensible 
estimates of concentration thresholds that separate the populations. Figure $3 \mathrm{~A}$ illustrates that if a normally distributed data set is sorted and plotted on a cumulative probability graph that the points fall on a straight line. For a straight line on such a plot, the mean of the data set is the concentration at the $50^{\text {th }}$ percentile, and the slope of the line is related to the standard deviation of the data. As discussed by Sinclair, significant deviations from this straight line are typically attributable to straightforward and explainable causes. Of particular note is the case where two populations are present (a low population and a high population). In this case (Figure 3B), the cumulative probability plot forms a bimodal curve that consists of two sections separated by an inflection region. The relative percentages of the two populations and the differences in the means and standard deviations determine the precise shape of the plot, but the plot can be explicitly and quantitatively represented as a mixture of the two populations.

Figure 3C is an actual example from Sinclair (1976) showing the method results for lead concentrations in rocks from 323 sample locations. In this example, both data sets are lognormally distributed. The upper (high concentration) line is interpreted to be sulfides with a mean concentration of $550 \mathrm{ppm}$ while the lower (low concentration) line is the background rock with a mean concentration of $32 \mathrm{ppm}$. The curved line represents the expected data distribution if the samples were $90 \%$ "background" rock and 10\% "contaminated" sulfide samples.

Thresholds to classify samples are typically set at the $\sim 98 \%$ confidence interval for the two populations, e.g. A (low) and B (high). If these overlap, the intersecting band is designated as a "combination of population A and B." The area beneath the band is designated as " $\mathrm{A}$ " and the area above the band is designated as "B". All of these methods are discussed in detail in Sinclair (1976), and are implemented in the computer program PROBPLOT (Stanley, 1987) distributed by the Association of Exploration Geochemists, and can be easily implemented in spreadsheets such as Microsoft EXCEL.

A summary of the advantages and disadvantages of probability plotting is provided below:

\begin{tabular}{|c|c|}
\hline \multicolumn{2}{|c|}{$\begin{array}{l}\text { Summary of advantages and disadvantages of using probability graphs for } \\
\text { geochemical data }\end{array}$} \\
\hline Advantages & Disadvantages \\
\hline $\begin{array}{l}\text { Simple rapid and easily understood method } \\
\text { to represent and interpret data }\end{array}$ & $\begin{array}{l}\text { Represents data as normal or lognormal } \\
\text { distribution (i.e., data might not } \\
\text { approximate such distributions) }\end{array}$ \\
\hline $\begin{array}{l}\text { Rapid estimation of parameters for normal } \\
\text { and lognormal distributions }\end{array}$ & $\begin{array}{l}\text { Requires a large amount of data (e.g., most } \\
\text { useful for datasets containing } 70 \text { to } 100 \\
\text { data points or more). }\end{array}$ \\
\hline $\begin{array}{l}\text { Compact representation of several data sets } \\
\text { and populations on a single diagram }\end{array}$ & $\begin{array}{l}\text { Tails of cumulative distribution are often } \\
\text { not well defined and require careful } \\
\text { interpretation to avoid bias. }\end{array}$ \\
\hline $\begin{array}{l}\text { Straightforward recognition, partitioning, } \\
\text { and threshold estimation for multiple } \\
\text { populations possible }\end{array}$ & $\begin{array}{l}\text { A small proportion of plots are not } \\
\text { interpretable using the procedures outlined } \\
\text { by Sinclair (1976) }\end{array}$ \\
\hline
\end{tabular}


Rapid identification of abnormalities in data set (e.g. truncation) possible and method insensitive to such abnormalities.
Low quality data can result in ambiguous or incorrect interpretation

Based upon the work of Sinclair and applications in the field on mineral exploration the analysis presented herein is a reasonable technical based estimate of mercury background in SRS groundwater. Two key/unique features of the work include: a) careful use of estimated values below the nominal detection limit, and $b$ ) interpretation of the data using graphical techniques that are insensitive to minor levels of data truncation and lack of prior information on which wells are contaminated and background. Nonetheless, the estimates are considered as preliminary because the analytical methods used were not optimized for analysis of samples at levels below the nominal detection limit. Also, the laboratory work was not performed in a clean room setting (as is done for specialized ultra high sensitivity methods). It is recommend that confirmation and improvement of the preliminary estimate be performed by using analysis with newer more sensitive methods (e.g. EPA Method 1631).

In order to properly apply order statistics and data partitioning techniques presented by Sinclair, the 518 "Estimated" values and the 856 "Non-Detect" values had to be associated. To facilitate handling this censorship of the data, the 518 estimated concentrations below the nominal detection limit were assumed to be representative of the "Non-Detect" samples (i.e., each actual estimate was assumed to represent approximately 2.6 samples for placement of the values on the probability graph). These data, combined with the measurements above the nominal detection limit, were sorted and used to prepare a cumulative probability graph. The geo-statistical analysis was performed in Microsoft EXCEL as described in Newman et al. (1989) - see method description for "order statistics." The results were checked using the PROBPLOT program (Stanley, 1987) distributed by the Association of Exploration Geochemists. 


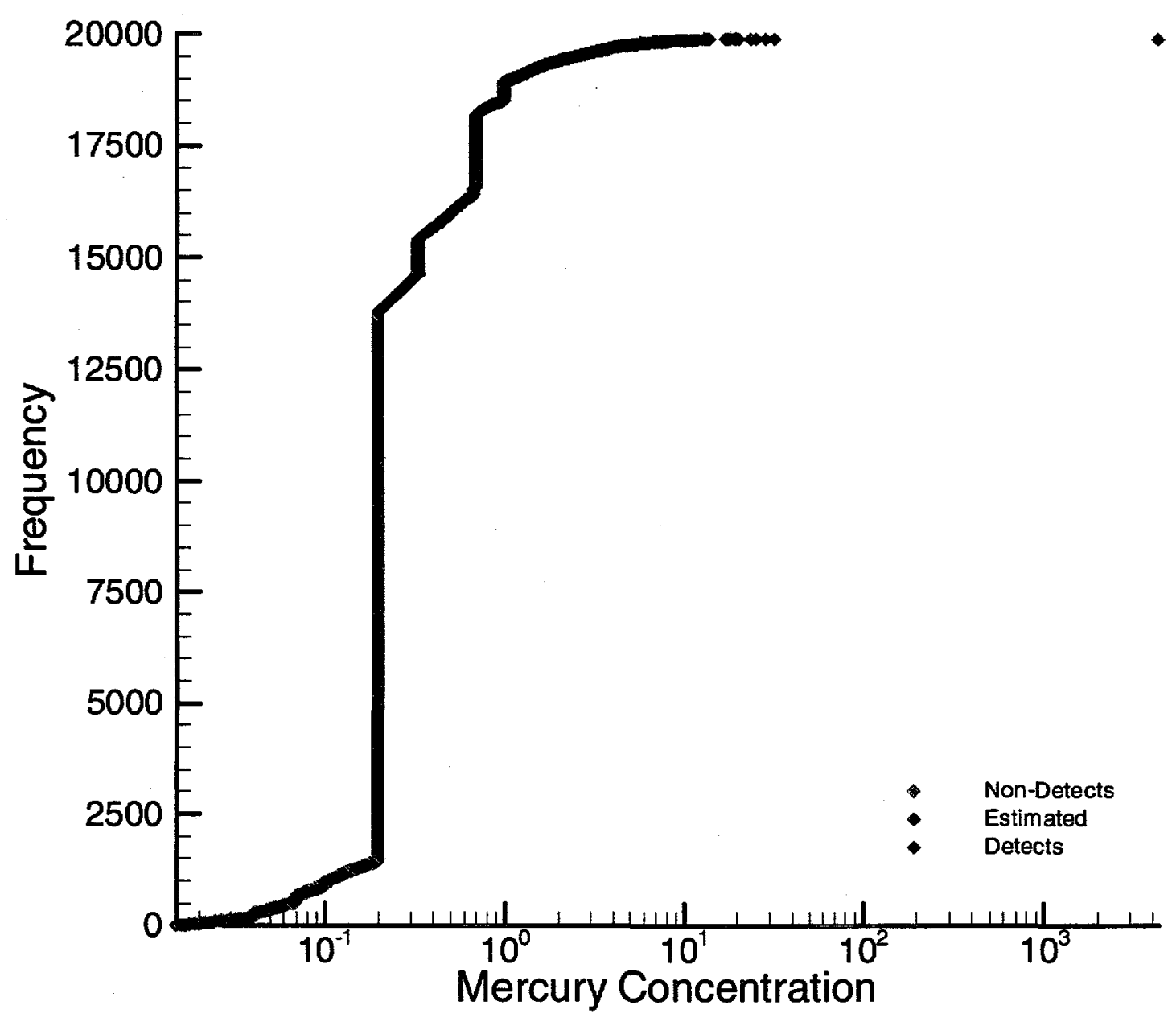

Figure 1: Cumulative Histogram of Mercury Concentrations from 19,917 Groundwater Measurements at the Savannah River Site including Estimated and Non-Detect values. 


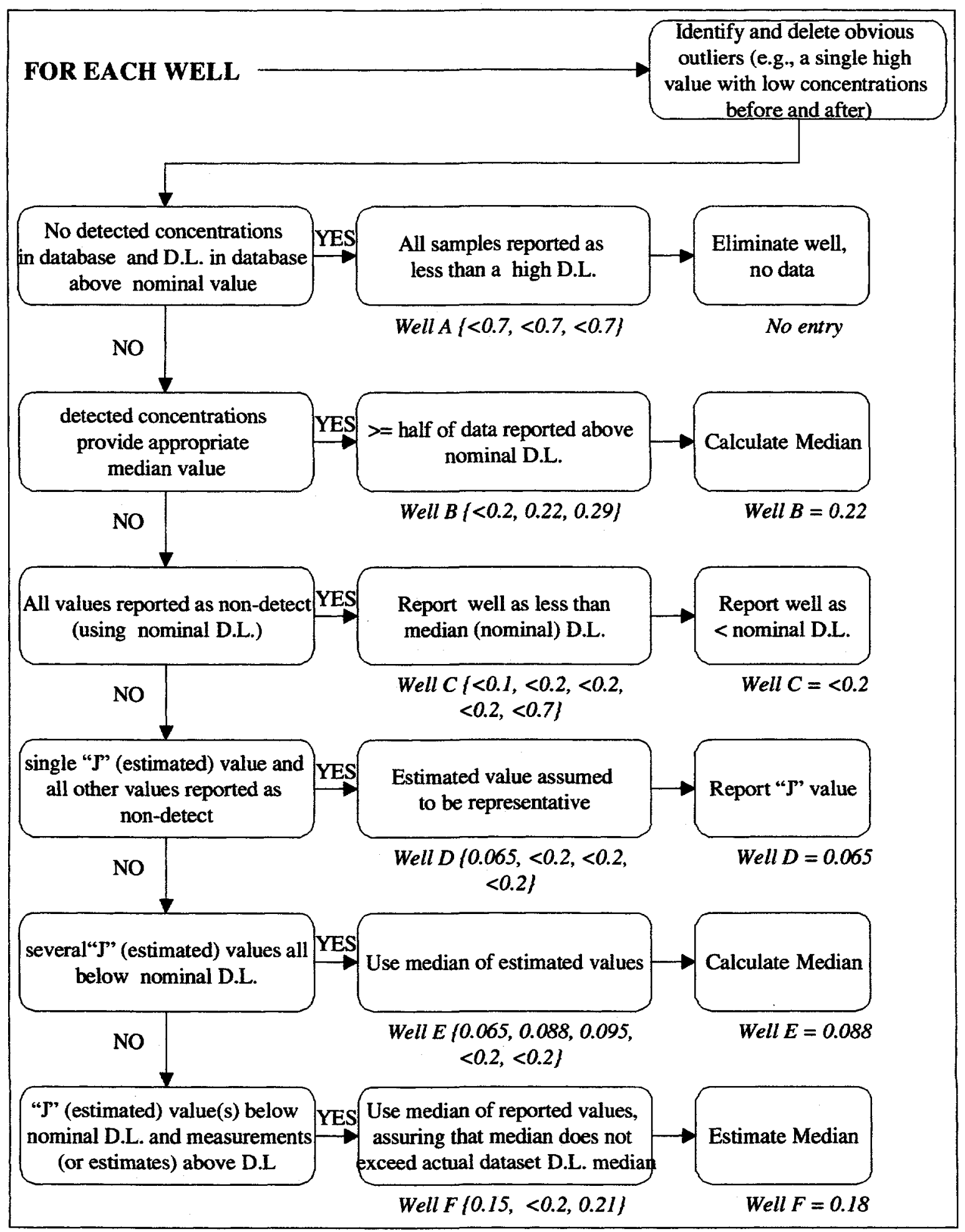

Figure 2: Flowchart Outlining Method Used to Obtain Best Estimate of Concentration Considering "Non-Detects and "Estimated" Values. 


\section{Figure 3A}

Normal distributions with different mems, but identical standand deviation plet as a set of perallet straigtu lines on probability paper. meens can be read where the lines intasen the sorh percentile.

Normal distributions with the same meen bue different standard Stminderd deviation is estimated by the slope of the line.

Normal distribution

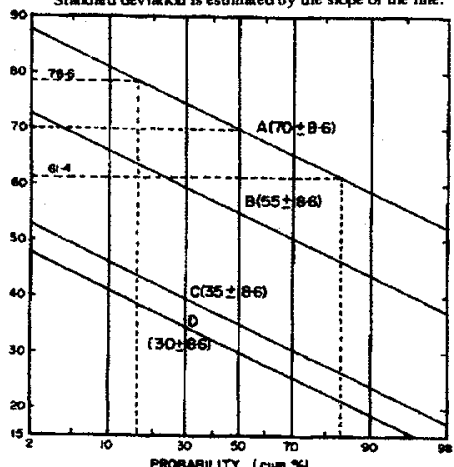
case, the lines irtersect at the soh pencertile.

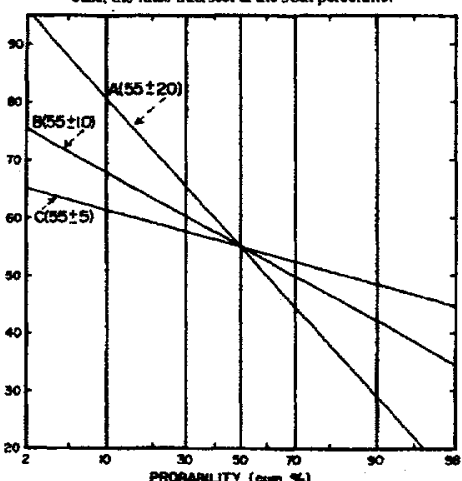

Figure 3B

Illustration of a probability grath of mixed populations. The black dass show conveniert arbirrary points on the curve and how the partiti regions representirg primarily $A$ or $B$. Points in the ceniral part, the region of over iap, $c$ an be

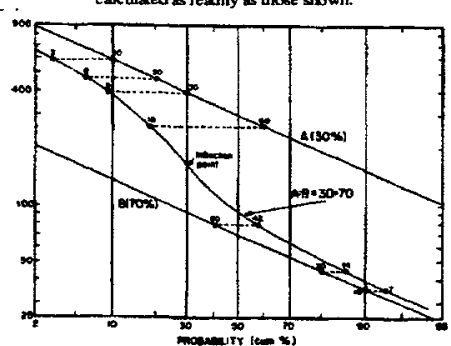

Figure 3C

Probibility Columbia. This dea ses ithustrates representing PB in sulfides and back pound-silicale rocks, respectively.

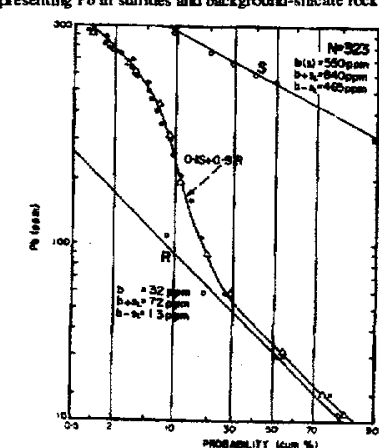

Figure 3: Application of Probability Plots in Identifying and Partitioning of two LogNormal Populations from Combined Data Sets. (Adopted from Sinclair, 1976). 


\section{Results and Analysis}

Using order statistics methods presented by Newman et al. (1989) with probability plotting and partitioning techniques presented by Sinclair (1976), the composite data set containing the best estimate of mercury concentration at the 1,724 locations was examined. The results of this examination are presented in Figure 4 . This figure presents the probability plot of mercury concentration for the 1,724 locations associated with the Savannah River Site. When plotted on the appropriate probability scale the data form a bimodal curve representative of two combined lognormal populations. The plotted data shows evidence of truncation by the observed flattening for mercury concentrations below $0.02 \mu \mathrm{g} / \mathrm{L}$. This flattening is expected based upon the limits of the analytical methods used to quantify the mercury concentrations. In reality, samples with trace mercury concentrations will be reported, and subsequently grouped, as "Non-detect", as measurement and detection in this range is difficult. The geo-statistical method employed equally distributes these values below the reported detection limit without any weighting. Statistical techniques are available to estimate and consider truncation effects, however application is often subjective. The authors believe that the careful identification and incorporation of the best estimate concentration for every well maintains data integrity and quality.

Visual inspection of the bimodal curve presented in Figure 4 indicates that the data can be represented as two populations and that an inflection point exists at a $\mathrm{z}$-score of about -1.2 . This indicates that approximately $90 \%$ of the data are associated with the "Background" population and that the remaining $10 \%$ are associated with the "Impacted" population. Using this initial estimate and the methods described by Sinclair (1976) the composite data set was rigorously partitioned using quantitative methods into an "Impacted" population and a "Background" population. Summary results of this analysis are presented in Table 1 and are also presented graphically in Figure 4. As shown the "Background" population has a mean concentration of $0.093 \mu \mathrm{g} / \mathrm{L}$ and the "Impacted" population has a mean concentration of $1.14 \mu \mathrm{g} / \mathrm{L}$.

Confidence intervals and the amount of population overlap were determined using the estimated mean and standard deviation information for each population. These calculations allow investigators to identify and compare individual well results so that a specific well can be classified into the appropriate population. The results from this analysis are summarized in Table 1 with specific well detail information enclosed in the Appendices. Figure 5 presents the location and distribution of the monitoring wells at the Savannah River Site and identifies the resulting population identified in this analysis.

The preceding analysis provides a reasonable upper bound on the estimated ambient, background concentration for mercury in the SRS groundwater system. Through the analysis several uncertainties were identified that could have an effect on this estimate. These uncertainties include possible elevated concentrations in wells due to interference from grout contamination, the potential for increased mercury concentrations resulting from laboratory contamination, and the use of data only from a relatively brief sampling window.

High pH values in grout contaminated wells can result in elevated concentrations of mercury in water sampled from these wells. Most natural mercury in SRS sediments was originally deposited as trace concentrations in minerals such as magnetite, feldspar, and apatite. Though concentrations of mercury in these phases at SRS are not known, mercury concentrations in similar phases of other rocks have ranged from tens to hundreds of parts per billion (Aidinyan et. 
al., 1963). The weathering of feldspar and magnetite in SRS sediments has produced clays and iron hydroxides and much of the original depositional mercury is probably associated with these fine-grained phases. Suspension of these phases as colloidal particles occurs at high $\mathrm{pH}$ values (Strom et. al., 1994) such as those caused by grout contamination. Typically, water in such wells has elevated turbidity and elevated concentrations of iron and aluminum from dispersal of clays and iron hydroxides. When this water is analyzed unfiltered (the case for compliance sampling at SRS) the colloidal particles and associated trace metals are analyzed as well, but are not differentiated from dissolved metals. Thus, inclusion of grout contaminated wells can bias the statistical analysis of mercury concentrations in groundwater toward higher values.

Bloom (1995) documented that "reported" mercury concentrations for "pristine" waters have declined significantly since the early 1970's. While this observation may reflect actual declines in environmental levels, Bloom (1995) suggests that improvements in sampling methods and that the use of clean-rooms have played a role in eliminating potential positive bias at very low concentrations. Because the values used in our estimate were not specifically performed to the clean-room recommendations, there is significant potential for bias in the result. In recent work however, Hall (1998) carefully examined potential analytical problems associated with determining "pristine" aqueous metal concentrations (including mercury). This study suggests that special cleaning methods may not be required in all cases.

As discussed in previous sections the data set developed and incorporated in this analysis was obtained from the GIMS production database of well concentration. Unfortunately the records in this database are limited in a historical perspective. Currently this database contains historical records from the early 1990's to present day. This is indicated by the period of record, October 1992 through August 1998, for the data set employed. This period post-dates most mercuryrelated activities at the Savannah River Site.

In relating the groundwater background to potential surface water impacts, two major uncertainties exist. These uncertainties relate to the surface and water groundwater interactions that occur in the hyporheic zone and the species of mercury present in the stream. Both of these uncertainties can best be managed by a limited amount of speciated analysis of both groundwater and surface water samples. The majority of the previous site investigations have only reported total recoverable mercury in both the groundwater and/or surface water. The AWQC criterion for mercury is based on toxicology studies with the assumption that all of the mercury in the stream is methyl mercury, the most toxic form, with a high bio-accumulation factor. Since mercury can exist in several forms in the environment, definitive data is needed on mercury species to support decisions regarding stream quality and discharge limits.

Table 1 Summary Information from Partitioning Analysis of Groundwater Mercury Concentration from 1,724 Monitoring Wells at the Savannah River Site.

\begin{tabular}{|l|c|c|c|}
\hline \multicolumn{1}{|c|}{ Population } & Mean & Size & $\begin{array}{c}\text { Confidence Interval } \\
(\mathbf{9 7 . 7 2 \% )}\end{array}$ \\
\hline Background & $0.093 \mu \mathrm{g} / \mathrm{L}$ & 439 & $0.021-0.407 \mu \mathrm{g} / \mathrm{L}$ \\
\hline Site Impacted & $1.14 \mu \mathrm{g} / \mathrm{L}$ & 157 & $0.16-8.06 \mu \mathrm{g} / \mathrm{L}$ \\
\hline Undetermined & $\mathrm{N} / \mathrm{A}$ & 272 & $0.16-0.407 \mu \mathrm{g} / \mathrm{L}$ \\
\hline Non-Detects & $<0.2 \mu \mathrm{g} / \mathrm{L}$ & 856 & $\mathrm{~N} / \mathrm{A}$ \\
\hline
\end{tabular}


Mercury in Groundwater at SRS

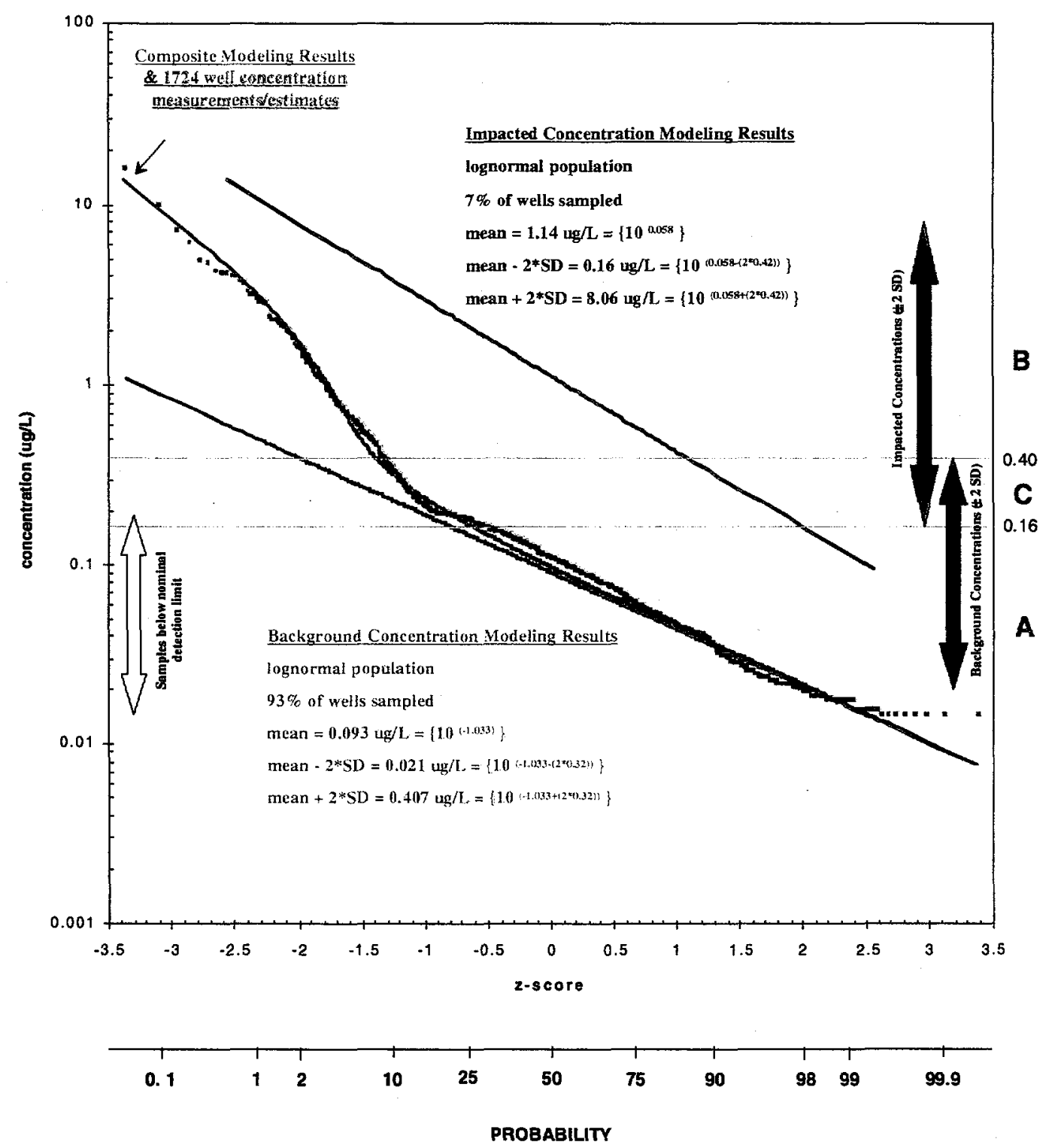

Figure 4: Log Probability Plot of Mercury Concentration from 1,724 Wells at the Savannah River Site. Populations partitioned into an Impacted Population and a Background Population. 


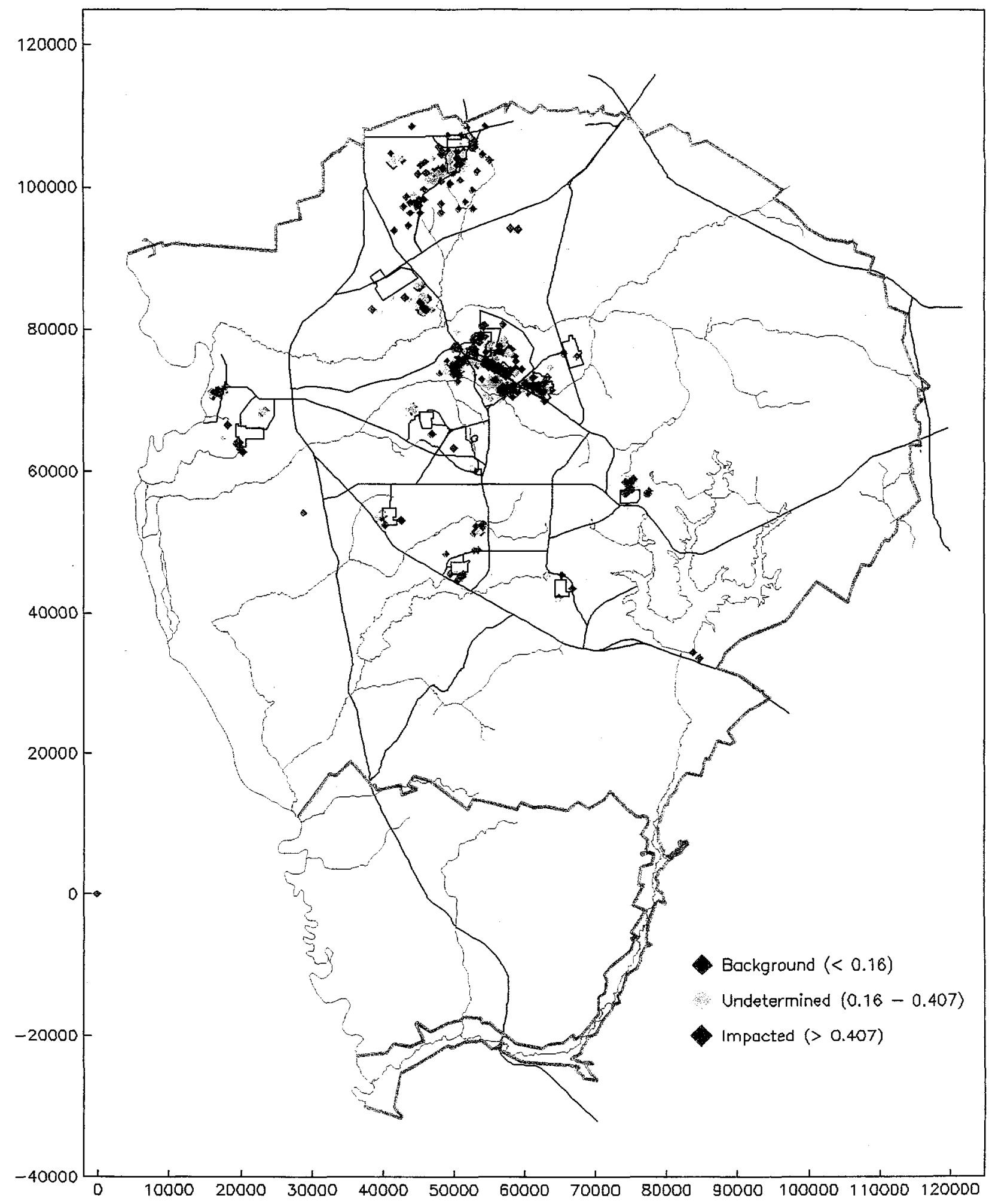

Figure 5: Map of the Savannah River Site Showing the Location and Distribution of Wells that are in the Background Population, in the Impacted Population, and whose population is Undetermined. 


\section{Recommendations and Conclusions}

Developing a technically defensible understanding of mercury in SRS groundwater and associated surface water is critical to supporting the environmental decision making process. Historical measurements from monitoring wells provide the basis for a preliminary estimate of background concentrations and contaminated concentrations in groundwater. This estimate, in turn may assist in related activities. For example, an upper bound on expected background stream concentrations may be possible based on background concentrations in the groundwater cropping out to these streams. Order statistics have been combined with probability plotting and partitioning techniques to estimate the background groundwater concentration for total recoverable mercury as $0.093 \mu \mathrm{g} / \mathrm{L}$.

Uncertainties in groundwater and surface water background estimates are best addressed by obtaining a limited amount of data using appropriate methods and suitable detection limits. The majority of previous data, both at SRS and offsite, have reported only "total recoverable mercury" at nominal detection limits of 0.1 to $0.2 \mu \mathrm{g} / \mathrm{L}$ (levels that are above expected levels). To be most useful, a future "special study" of mercury should include total mercury and mercury speciation analysis of selected samples in both groundwater and surface water at relevant detection limits (e.g., $<0.012 \mu \mathrm{g} / \mathrm{L}$ ). Mercury exists in several forms in the environment, each with varying toxicity/risk. This makes the collection of definitive speciation information central to future technical and regulatory proposals. The resulting data are needed to allow decisions to be based on actual concentrations rather than on a periodic detection at 0.1 to $0.2 \mu \mathrm{g} / \mathrm{L}$. 


\section{References}

Aidinyan, N.Kh., L.L. Shilin, and G.A. Belavskaya, 1963, O raspredelenii rtuti v porodakh I mineralakh Khibinskovo Massiva (Concerning the distribution of mercury in the rocks and minerals of the Khibinsky Massif), Tr. Inst. Geol. Rudn. Mestorozhd, Mineralog. i Geokhim., v. 99, p. 16-25.

Anderson, A. (1970). On the Geochemical Behavior of Mercury. Grundforbattring 23:5, pp 3144.

Bloom, N. (1995). Ultraclean sample handling. Environmental Laboratory, March/April 1995, pp20-25.

Denham, Miles E. and Richard s. Strom. (1994) Mercury Speciation Analyses of H-Area Soil Samples. Inter-Office Memorandum SRT-ESS-94-310, Westinghouse Savannah River Company, Aiken, South Carolina 29808.

EPA, (1992). Guidance for Data Usability in Risk Assessment (Part A). Report PB92-963356, April 1992, Environmental Protection Agency, Office of Emergency and Remedial Response, Washington D.C.

GMS (1997). Geochemical Information Management System Users Manual, Westinghouse Savannah River Company, Aiken, South Carolina 29808.

Hall, G.E. (1998). Relative Concentration levels observed in different types of bottles used to collect water samples. EXPLORE: Newsletter of the Association of Exploration Geochemists, November 1998, pg 1-7.

Lindberg, S. and Vermette, S. 1995. Workshop on Sampling Mercury in Precipitation for the National Atmospheric Deposition Program. Atmospheric Environment. 29, 1219-1220.

Looney, B.B., C.A. Eddy, M. Ramdeen, J. Pickett, V. Rogers, M.T. Scott, and P.A. Shirley (1990). Geochemical and Physical Properties of Soils and Shallow Sediments at the Savannah River Site (U). Technical Report WSRC-RP-90-1031, August 31, 1990, Westinghouse Savannah River Company, Aiken, South Carolina 29808.

Looney, Brian B. (1994) Scoping Calculation on Potential Mercury Migration from Spill (Process Water Entry Flange) Near Building 672T, Inter-Office Memorandum SRT-ESS94-124, Westinghouse Savannah River Company, Aiken, South Carolina 29808.

Newman, M. C., P. M. Dixon, B. B. Looney and J. E. Pinder III, 1989. "Estimating Mean and Variance for Environmental Samples with Below Detection Limit Observations." Water Resources Bulletin, 25:4, pp 905-916.

Newman, M.C. and A. Messier (1994). Mercury Bioaccumulation in Mosquitofish (Gambusia holbrooki) Sampled on and around the Savannah River Site. Technical Report SREL-48 UC-66E, Biogeochemical Ecology Division, Savannah River Ecology Laboratory, Drawer E, Aiken, South Carolina 29802.

Sinclair, A.J. (1989). Applications of Probability Graphs in Mineral Exploration. Special Volume No. 4, The Association of Exploration Geochemists, P.O. Box 523, Metropolitan Toronto, Rexdale, Ontario M9W 5L4, Canada. 
Stanley, C. R., 1987. PROBPLOT: An Interactive Computer Program to Fit Mixtures of Normal (or Lognormal) Distributions with Maximum Likelihood Optimization Procedures. Special Volume No. 14, Association of Exploration Geochemists, Rexdale Ontario, Canada.

Strom, R.N., B.B. Looney, J.C. Seaman, P.M. Bertsch, and W.P. Miller, 1994, Summary Report and Report; Physicochemical and Mineralogical Controls on Colloid Migration and Deposition within Sediments on the Savannah River Site (U), WSRC-RP-94-276, Westinghouse Savannah River Company, Aiken, SC 29808.

USGS (1998) Groundwater and Surface Water: A Single Resource. U.S. Geological Survey Circular 1139, U.S. Geological Survey, Branch of Information Services, Box 25286, Denver, Colorado 80225-0286.

Kvartek, E.J. , W.H. Carlton, M.E. Denham, L. Eldridge, and M.C. Newman (1994). Assessment of Mercury in the Savannah River Site Environment (U). Technical Report WSRC-TR94-0218ET, September 1994, Westinghouse Savannah River Company, Aiken, South Carolina 29808.

Vermette, S., Lindberg, S., and Bloom, N. (1995). Field Tests for a Regional Mercury Deposition Network - Sampling Design and Preliminary Test Results. Atmospheric Environment. 29, 1247-1251.

Welker, M. and Vermette, S.J., (1996). Mercury Deposition Network: QA/QC Protocols. Paper 96-RP129.01, Proceedings of the $89^{\text {th }}$ Annual Meeting of the Air and Waste Management Association, A\&WMA, Pittsburgh, PA.

WSRC, (1997a). The Savannah River Site's Groundwater Monitoring Program First Quarter 1997 (U), Report ESH-EMS-970488, Environmental Monitoring Section, The Environmental Protection Department, Westinghouse Savannah River Company, Aiken, South Carolina 29808.

WSRC, (1997b). The Savannah River Site's Groundwater Monitoring Program Second Quarter 1997 (U), Report ESH-EMS-970489, Environmental Monitoring Section, The Environmental Protection Department, Westinghouse Savannah River Company, Aiken, South Carolina 29808.

WSRC, (1997c). The Savannah River Site's Groundwater Monitoring Program Third Quarter 1997 (U), Report ESH-EMS-970490, Environmental Monitoring Section, The Environmental Protection Department, Westinghouse Savannah River Company, Aiken, South Carolina 29808.

WSRC, (1997d). The Savannah River Site's Groundwater Monitoring Program Fourth Quarter 1997 (U), Report ESH-EMS-970491, Environmental Monitoring Section, The Environmental Protection Department, Westinghouse Savannah River Company, Aiken, South Carolina 29808. 
Appendix A: Estimated Median Concentrations for SRS Groundwater Wells Associated with "Background" Population.

(total mercury $<0.16 \mathrm{ug} / \mathrm{L}$ ) 


\begin{tabular}{|c|c|c|c|c|}
\hline \multicolumn{5}{|c|}{ Wells ( $n=439$ ) with median mercury concentration estimated $<0.16 \mathrm{ug} / \mathrm{L}$} \\
\hline well & qualifier & result & units & analyte_name \\
\hline $\mathrm{ABP} 1 \mathrm{~A}$ & $\mathrm{~J}$ & 0.106 & UGL & Mercury, total recoverable \\
\hline $\mathrm{ABP} 3$ & $\mathrm{~J}$ & 0.133 & UGL & Mercury, total recoverable \\
\hline $\mathrm{ABP} 8 \mathrm{D}$ & $\mathrm{J}$ & 0.036 & UGL & Mercury, total recoverable \\
\hline ABP 9C & $\mathrm{J}$ & 0.026 & UGL & Mercury, total recoverable \\
\hline ABP 9D & $\mathrm{J}$ & 0.06 & UGL & Mercury, total recoverable \\
\hline ABP 10D & $\mathrm{J}$ & 0.062 & UGL & Mercury, total recoverable \\
\hline AMB 5 & $\mathbf{J}$ & 0.07 & UGL & Mercury, total recoverable \\
\hline AMB 6 & $\mathrm{~J}$ & 0.05 & UGL & Mercury, total recoverable \\
\hline AMB 7 & $\mathbf{J}$ & 0.064 & UGL & Mercury, total recoverable \\
\hline AMB 7A & $\mathrm{J}$ & 0.067 & UGL & Mercury, total recoverable \\
\hline $\mathrm{AMB} 7 \mathrm{~B}$ & $\mathrm{~J}$ & 0.02 & UGL & Mercury, total recoverable \\
\hline AMB 8D & $\mathrm{J}$ & 0.046 & UGL & Mercury, total recoverable \\
\hline AMB 9D & $\mathbf{J}$ & 0.015 & UGL & Mercury, total recoverable \\
\hline AMB 10A & $\mathbf{J}$ & 0.09 & UGL & Mercury, total recoverable \\
\hline AMB 10DD & $\mathbf{J}$ & 0.021 & UGL & Mercury, total recoverable \\
\hline $\mathrm{AMB} 11 \mathrm{~B}$ & $\mathbf{J}$ & 0.018 & UGL & Mercury, total recoverable \\
\hline AMB 15D & $\mathrm{J}$ & 0.058 & UGL & Mercury, total recoverable \\
\hline AMB 16D & $\mathrm{J}$ & 0.046 & UGL & Mercury, total recoverable \\
\hline AMB 17A & $\mathrm{J}$ & 0.0565 & UGL & Mercury, total recoverable \\
\hline AMB 18A & $\mathrm{J}$ & 0.0675 & UGL & Mercury, total recoverable \\
\hline AMB 18C & $\mathrm{J}$ & 0.046 & UGL & Mercury, total recoverable \\
\hline ASB 1A & $\mathrm{J}$ & 0.086 & UGL & Mercury, total recoverable \\
\hline ASB 2AR & $\mathbf{J}$ & 0.084 & UGL & Mercury, total recoverable \\
\hline ASB 3AR & $\mathrm{J}$ & 0.098 & UGL & Mercury, total recoverable \\
\hline ASB 4 & & 0.1315 & UGL & Mercury, total recoverable \\
\hline ASB 5C & $\mathbf{J}$ & 0.088 & UGL & Mercury, total recoverable \\
\hline ASB $6 \mathrm{~A}$ & $\mathrm{~J}$ & 0.1285 & UGL & Mercury, total recoverable \\
\hline ASB 6AA & $\mathrm{J}$ & 0.083 & UGL & Mercury, total recoverable \\
\hline ASB $6 C$ & $\mathrm{~J}$ & 0.13 & UGL & Mercury, total recoverable \\
\hline ASB 8 & $\mathrm{~J}$ & 0.073 & UGL & Mercury, total recoverable \\
\hline ASB 8A & $\mathrm{J}$ & 0.046 & UGL & Mercury, total recoverable \\
\hline ASB 8B & $\mathbf{J}$ & 0.078 & UGL & Mercury, total recoverable \\
\hline ASB 8TA & $\mathrm{J}$ & 0.13 & UGL & Mercury, total recoverable \\
\hline ASB 9 & $\mathbf{J}$ & 0.067 & UGL & Mercury, total recoverable \\
\hline ASB 9B & $\mathrm{J}$ & 0.1585 & UGL & Mercury, total recoverable \\
\hline BGO 1D & $\mathrm{J}$ & 0.068 & UGL & Mercury, total recoverable \\
\hline BGO 2D & $\mathrm{J}$ & 0.032 & UGL & Mercury, total recoverable \\
\hline BGO 3DR & $\mathrm{J}$ & 0.0425 & UGL & Mercury, total recoverable \\
\hline BGO 4D & $\mathbf{J}$ & 0.029 & UGL & Mercury, total recoverable \\
\hline BGO 6A & $\mathrm{J}$ & 0.023 & UGL & Mercury, total recoverable \\
\hline BGO 8AR & $\mathrm{J}$ & 0.03 & UGL & Mercury, total recoverable \\
\hline BGO 8C & $\mathrm{J}$ & 0.03 & UGL & Mercury, total recoverable \\
\hline BGO 8D & $\mathbf{J}$ & 0.042 & UGL & Mercury, total recoverable \\
\hline BGO 9D & $\mathbf{J}$ & 0.031 & UGL & Mercury, total recoverable \\
\hline BGO 10AA & $\mathbf{J}$ & 0.099 & UGL & Mercury, total recoverable \\
\hline BGO 10AR & $\mathbf{J}$ & 0.029 & UGL & Mercury, total recoverable \\
\hline BGO 10DR & $\mathrm{J}$ & 0.075 & UGL & Mercury, total recoverable \\
\hline BGO 11DR & $\mathrm{J}$ & 0.033 & UGL & Mercury, total recoverable \\
\hline BGO 12AX & $\mathrm{J}$ & 0.14 & UGL & Mercury, total recoverable \\
\hline BGO 12CX & $\mathrm{J}$ & 0.132 & UGL & Mercury, total recoverable \\
\hline BGO 14AR & $\mathrm{J}$ & 0.101 & UGL & Mercury, total recoverable \\
\hline BGO 14CR & $\mathrm{J}$ & 0.142 & UGL & Mercury, total recoverable \\
\hline BGO 14DR & $\mathbf{J}$ & 0.077 & UGL & Mercury, total recoverable \\
\hline
\end{tabular}




\begin{tabular}{|c|c|c|c|c|}
\hline \multicolumn{5}{|c|}{ Wells $(n=439$ ) with median mercury concentration estimated $<0.16 \mathrm{ug} / \mathrm{L}$} \\
\hline well & qualifier & result & units & analyte_name \\
\hline $\mathrm{BGO} 16 \mathrm{~B}$ & $\mathbf{J}$ & 0.028 & UGL & Mercury, total recoverable \\
\hline BGO 20A & $\mathbf{J}$ & 0.034 & UGL & Mercury, total recoverable \\
\hline $\mathrm{BGO} 20 \mathrm{AA}$ & $\mathbf{J}$ & 0.099 & UGL & Mercury, total recoverable \\
\hline $\mathrm{BGO} 20 \mathrm{~B}$ & $\mathbf{J}$ & 0.109 & UGL & Mercury, total recoverable \\
\hline $\mathrm{BGO} 20 \mathrm{C}$ & $\mathbf{J}$ & 0.111 & UGL & Mercury, total recoverable \\
\hline $\mathrm{BGO} 20 \mathrm{D}$ & $\mathbf{J}$ & 0.104 & UGL & Mercury, total recoverable \\
\hline $\mathrm{BGO} 21 \mathrm{D}$ & $\mathbf{J}$ & 0.016 & UGL & Mercury, total recoverable \\
\hline BGO 23D & $\mathbf{J}$ & 0.0535 & UGL & Mercury, total recoverable \\
\hline $\mathrm{BGO} 27 \mathrm{D}$ & $J$ & 0.045 & UGL & Mercury, total recoverable \\
\hline BGO 30C & $\mathbf{J}$ & 0.087 & UGL & Mercury, total recoverable \\
\hline $\mathrm{BGO} 32 \mathrm{D}$ & $\mathbf{J}$ & 0.078 & UGL & Mercury, total recoverable \\
\hline $\mathrm{BGO} 33 \mathrm{D}$ & $\mathrm{J}$ & 0.1105 & UGL & Mercury, total recoverable \\
\hline $\mathrm{BGO} 35 \mathrm{C}$ & $\mathrm{J}$ & 0.0665 & UGL & Mercury, total recoverable \\
\hline $\mathrm{BGO} 35 \mathrm{D}$ & $J$ & 0.137 & UGL & Mercury, total recoverable \\
\hline $\mathrm{BGO} 37 \mathrm{D}$ & $\mathrm{J}$ & 0.071 & UGL & Mercury, total recoverable \\
\hline $\mathrm{BGO} 39 \mathrm{~A}$ & $\mathbf{J}$ & 0.059 & UGL & Mercury, total recoverable \\
\hline $\mathrm{BGO} 44 \mathrm{AA}$ & $\mathbf{J}$ & 0.135 & UGL & Mercury, total recoverable \\
\hline $\mathrm{BGO} 46 \mathrm{C}$ & $\mathbf{J}$ & 0.034 & UGL & Mercury, total recoverable \\
\hline BGO 49C & $\bar{J}$ & 0.049 & UGL & Mercury, total recoverable \\
\hline $\mathrm{BGO} 49 \mathrm{D}$ & $\mathbf{J}$ & 0.022 & UGL & Mercury, total recoverable \\
\hline BGO 50A & $\mathbf{J}$ & 0.055 & UGL & Mercury, total recoverable \\
\hline $\mathrm{BGO} 51 \mathrm{~A}$ & $\mathbf{J}$ & 0.082 & UGL & Mercury, total recoverable \\
\hline BGO 51AA & $\mathbf{J}$ & 0.151 & UGL & Mercury, total recoverable \\
\hline BGO 51B & $\mathbf{J}$ & 0.073 & UGL & Mercury, total recoverable \\
\hline BGO 51C & $\mathbf{J}$ & 0.032 & UGL & Mercury, total recoverable \\
\hline BGO 51D & $\mathbf{J}$ & 0.101 & UGL & Mercury, total recoverable \\
\hline BGO 52A & $\mathrm{J}$ & 0.093 & UGL & Mercury, total recoverable \\
\hline $\mathrm{BGO} 52 \mathrm{AA}$ & $\mathbf{J}$ & 0.04 & UGL & Mercury, total recoverable \\
\hline $\mathrm{BGO} 52 \mathrm{~B}$ & $\mathrm{~J}$ & 0.153 & UGL & Mercury, total recoverable \\
\hline BGO 52D & $\mathrm{J}$ & 0.045 & UGL & Mercury, total recoverable \\
\hline $\mathrm{BGO} 53 \mathrm{~A}$ & $\mathrm{~J}$ & 0.088 & UGL & Mercury, total recoverable \\
\hline $\mathrm{BGO} 53 \mathrm{~B}$ & $\mathrm{~J}$ & 0.085 & UGL & Mercury, total recoverable \\
\hline $\mathrm{BGX} 2 \mathrm{~B}$ & $\mathrm{~J}$ & 0.015 & UGL & Mercury, total recoverable \\
\hline $\mathrm{BGX} 3 \mathrm{D}$ & $\mathrm{J}$ & 0.063 & UGL & Mercury, total recoverable \\
\hline $\mathrm{BGX} 4 \mathrm{C}$ & $\mathrm{J}$ & 0.096 & UGL & Mercury, total recoverable \\
\hline $\mathrm{BGX} 4 \mathrm{D}$ & $\mathrm{J}$ & 0.093 & UGL & Mercury, total recoverable \\
\hline $\mathrm{BGX} 5 \mathrm{D}$ & $\mathrm{J}$ & 0.028 & UGL & Mercury, total recoverable \\
\hline $\mathrm{BGX} 12 \mathrm{C}$ & $\mathrm{J}$ & 0.045 & UGL & Mercury, total recoverable \\
\hline $\mathrm{BGX} 12 \mathrm{D}$ & $\mathrm{J}$ & 0.042 & UGL & Mercury, total recoverable \\
\hline BRR 1D & $\mathrm{J}$ & 0.077 & UGL & Mercury, total recoverable \\
\hline BRR 2D & $\mathrm{J}$ & 0.063 & UGL & Mercury, total recoverable \\
\hline BRR 3D & $\mathrm{J}$ & 0.0955 & UGL & Mercury, total recoverable \\
\hline BRR 4D & $J$ & 0.054 & UGL & Mercury, total recoverable \\
\hline BRR 5D & $\mathrm{J}$ & 0.108 & UGL & Mercury, total recoverable \\
\hline BRR 6B & $\mathrm{J}$ & 0.0595 & UGL & Mercury, total recoverable \\
\hline BRR 6C & $\mathrm{J}$ & 0.086 & UGL & Mercury, total recoverable \\
\hline BRR 6D & $\mathrm{J}$ & 0.0525 & UGL & Mercury, total recoverable \\
\hline BRR 7D & $\mathrm{J}$ & 0.095 & UGL & Mercury, total recoverable \\
\hline BRR 8B & $J$ & 0.15 & UGL & Mercury, total recoverable \\
\hline BRR 8C & $\mathrm{J}$ & 0.142 & UGL & Mercury, total recoverable \\
\hline BRR 8D & $\mathrm{J}$ & 0.127 & UGL & Mercury, total recoverable \\
\hline CBR 1D & $\mathrm{J}$ & 0.0485 & UGL & Mercury, total recoverable \\
\hline CBR 4D & $\mathrm{J}$ & 0.046 & UGL & Mercury, total recoverable \\
\hline
\end{tabular}




\begin{tabular}{|c|c|c|c|c|}
\hline \multicolumn{5}{|c|}{ Wells $(n=439)$ with median mercury concentration estimated $<0.16 \mathrm{ug} / \mathrm{L}$} \\
\hline well & qualifier & result & units & analyte_name \\
\hline CCB 1 & $\mathrm{~J}$ & 0.061 & UGL & Mercury, total recoverable \\
\hline CCB 2 & $\mathrm{~J}$ & 0.15 & UGL & Mercury, total recoverable \\
\hline CCB 3 & $\mathrm{~J}$ & 0.092 & UGL & Mercury, total recoverable \\
\hline CMP 8 & $\mathrm{~J}$ & 0.1045 & UGL & Mercury, total recoverable \\
\hline CMP 8A & $\mathrm{J}$ & 0.076 & UGL & Mercury, total recoverable \\
\hline CMP 8B & $J$ & 0.073 & UGL & Mercury, total recoverable \\
\hline CMP 10B & $\mathbf{J}$ & 0.119 & UGL & Mercury, total recoverable \\
\hline CMP 10C & $\mathbf{J}$ & 0.055 & UGL & Mercury, total recoverable \\
\hline CMP 10D & $\mathrm{J}$ & 0.023 & UGL & Mercury, total recoverable \\
\hline CMP 12 & $\mathbf{J}$ & 0.041 & UGL & Mercury, total recoverable \\
\hline CMP 12A & $\mathbf{J}$ & 0.106 & UGL & Mercury, total recoverable \\
\hline CMP 12B & $\mathbf{J}$ & 0.079 & UGL & Mercury, total recoverable \\
\hline CMP 13 & $\mathbf{J}$ & 0.0985 & UGL & Mercury, total recoverable \\
\hline CMP 13B & $J$ & 0.147 & UGL & Mercury, total recoverable \\
\hline CMP 13D & $J$ & 0.064 & UGL & Mercury, total recoverable \\
\hline CMP 15A & $\mathbf{J}$ & 0.093 & UGL & Mercury, total recoverable \\
\hline CMP 15B & $\mathbf{J}$ & 0.143 & UGL & Mercury, total recoverable \\
\hline CMP 15C & $\mathbf{J}$ & 0.091 & UGL & Mercury, total recoverable \\
\hline CMP 16B & $\mathbf{J}$ & 0.127 & UGL & Mercury, total recoverable \\
\hline CMP 30C & $\mathrm{J}$ & 0.065 & UGL & Mercury, total recoverable \\
\hline CMP 30D & $\mathrm{J}$ & 0.0985 & UGL & Mercury, total recoverable \\
\hline CMP 31B & $\mathrm{J}$ & 0.125 & UGL & Mercury, total recoverable \\
\hline CMP 32C & $\mathrm{J}$ & 0.087 & UGL & Mercury, total recoverable \\
\hline CMP 32D & $\mathrm{J}$ & 0.0885 & UGL & Mercury, total recoverable \\
\hline CRP 2 & $\mathrm{J}$ & 0.0555 & UGL & Mercury, total recoverable \\
\hline CRP $3 \mathrm{C}$ & $\mathrm{J}$ & 0.135 & UGL & Mercury, total recoverable \\
\hline CRP 3D & $\mathbf{J}$ & 0.1185 & UGL & Mercury, total recoverable \\
\hline CRP 4 & $\mathbf{J}$ & 0.154 & UGL & Mercury, total recoverable \\
\hline CRP 5D & $J$ & 0.12 & UGL & Mercury, total recoverable \\
\hline CRP 6D & $\mathrm{J}$ & 0.106 & UGL & Mercury, total recoverable \\
\hline CRP 9D & $\mathrm{J}$ & 0.146 & UGL & Mercury, total recoverable \\
\hline CSB 2C & $\mathrm{J}$ & 0.06 & UGL & Mercury, total recoverable \\
\hline DCB 18A & $\mathrm{J}$ & 0.095 & UGL & Mercury, total recoverable \\
\hline DCB 18B & $\mathrm{J}$ & 0.1 & UGL & Mercury, total recoverable \\
\hline DCB 22A & $\mathbf{J}$ & 0.11 & UGL & Mercury, total recoverable \\
\hline DOB 1 & $\mathrm{~J}$ & 0.049 & UGL & Mercury, total recoverable \\
\hline DOB 2 & $\mathrm{~J}$ & 0.1375 & UGL & Mercury, total recoverable \\
\hline DOB 10 & $\mathrm{~J}$ & 0.115 & UGL & Mercury, total recoverable \\
\hline DOB 12 & $\mathbf{J}$ & 0.05 & UGL & Mercury, total recoverable \\
\hline DOB 16 & $\mathbf{J}$ & 0.09 & UGL & Mercury, total recoverable \\
\hline FAB 2 & $\mathbf{J}$ & 0.05 & UGL & Mercury, total recoverable \\
\hline FAC $11 \mathrm{C}$ & $\mathbf{J}$ & 0.08 & UGL & Mercury, total recoverable \\
\hline FAL 1 & $\mathbf{J}$ & 0.0655 & UGL & Mercury, total recoverable \\
\hline FAL 2 & $\mathbf{J}$ & 0.061 & UGL & Mercury, total recoverable \\
\hline FCA 10A & $\mathbf{J}$ & 0.075 & UGL & Mercury, total recoverable \\
\hline FCB 4 & $\mathrm{~J}$ & 0.09 & UGL & Mercury, total recoverable \\
\hline FIN 2TK & $J$ & 0.154 & UGL & Mercury, total recoverable \\
\hline FRB 4 & $\mathbf{J}$ & 0.06 & UGL & Mercury, total recoverable \\
\hline FSB 76 & $\mathbf{J}$ & 0.029 & UGL & Mercury, total recoverable \\
\hline FSB $78 \mathrm{C}$ & $\mathbf{J}$ & 0.0805 & UGL & Mercury, total recoverable \\
\hline FSB 79B & $\mathbf{J}$ & 0.085 & UGL & Mercury, total recoverable \\
\hline FSB 87C & $\mathbf{J}$ & 0.157 & UGL & Mercury, total recoverable \\
\hline FSB 96AR & $\mathbf{J}$ & 0.033 & UGL & Mercury, total recoverable \\
\hline
\end{tabular}




\begin{tabular}{|c|c|c|c|c|}
\hline \multicolumn{5}{|c|}{ Wells $(n=439)$ with median mercury concentration estimated $<0.16 \mathrm{ug} / \mathrm{L}$} \\
\hline well & qualifier & result & units & analyte_name \\
\hline FSB 97A & $\mathrm{J}$ & 0.05 & UGL & Mercury, total recoverable \\
\hline FSB 97C & $\mathrm{J}$ & 0.056 & UGL & Mercury, total recoverable \\
\hline FSB 97D & $\mathrm{J}$ & 0.129 & UGL & Mercury, total recoverable \\
\hline FSB 98AR & $\mathrm{J}$ & 0.086 & UGL & Mercury, total recoverable \\
\hline FSB 98D & $J$ & 0.154 & UGL & Mercury, total recoverable \\
\hline FSB 99C & $\mathrm{J}$ & 0.156 & UGL & Mercury, total recoverable \\
\hline FSB104C & $\mathbf{J}$ & 0.114 & UGL & Mercury, total recoverable \\
\hline FSB105DR & $J$ & 0.038 & UGL & Mercury, total recoverable \\
\hline FSB108D & $\mathbf{J}$ & 0.064 & UGL & Mercury, total recoverable \\
\hline FSB109D & $\mathrm{J}$ & 0.063 & UGL & Mercury, total recoverable \\
\hline FSB110C & $\mathrm{J}$ & 0.026 & UGL & Mercury, total recoverable \\
\hline FSB 111C & $\mathbf{J}$ & 0.058 & UGL & Mercury, total recoverable \\
\hline FSB111D & $J$ & 0.129 & UGL & Mercury, total recoverable \\
\hline FSB112A & $\mathrm{J}$ & 0.032 & UGL & Mercury, total recoverable \\
\hline FSB 113D & $J$ & 0.024 & $\mathrm{UGL}$ & Mercury, total recoverable \\
\hline FSB 114C & & 0.067 & UGL & Mercury, total recoverable \\
\hline FSB116C & $J$ & 0.119 & UGL & Mercury, total recoverable \\
\hline FSB120C & $\mathrm{J}$ & 0.032 & UGL & Mercury, total recoverable \\
\hline FSB121DR & $\mathrm{J}$ & 0.054 & UGL & Mercury, total recoverable \\
\hline FSB122C & $\mathrm{J}$ & 0.137 & UGL & Mercury, total recoverable \\
\hline FSB122D & $\mathrm{J}$ & 0.137 & UGL & Mercury, total recoverable \\
\hline FSL 1D & $\mathrm{J}$ & 0.064 & UGL & Mercury, total recoverable \\
\hline FSL 2D & $\mathrm{J}$ & 0.056 & UGL & Mercury, total recoverable \\
\hline FSL 5D & $\mathrm{J}$ & 0.048 & UGL & Mercury, total recoverable \\
\hline FSL 8D & $\mathrm{J}$ & 0.115 & UGL & Mercury, total recoverable \\
\hline FSS 3D & $J$ & 0.143 & UGL & Mercury, total recoverable \\
\hline FTF 16 & $\mathbf{J}$ & 0.152 & UGL & Mercury, total recoverable \\
\hline HAA 1D & $\mathrm{J}$ & 0.08 & UGL & Mercury, total recoverable \\
\hline HAA 1TA & $\mathrm{J}$ & 0.129 & UGL & Mercury, total recoverable \\
\hline $\mathrm{HCA} 2$ & $\mathrm{~J}$ & 0.067 & UGL & Mercury, total recoverable \\
\hline $\mathrm{HCA} 3$ & $\mathrm{~J}$ & 0.0975 & UGL & Mercury, total recoverable \\
\hline HCA 4AA & $\mathrm{J}$ & 0.13 & UGL & Mercury, total recoverable \\
\hline $\mathrm{HCA} 4 \mathrm{~B}$ & $\mathrm{~J}$ & 0.0945 & UGL & Mercury, total recoverable \\
\hline HCB 1 & $\mathbf{J}$ & 0.08 & UGL & Mercury, total recoverable \\
\hline HHP 1D & $\mathrm{J}$ & 0.1 & UGL & Mercury, total recoverable \\
\hline HR3 13 & $\mathrm{~J}$ & 0.1175 & UGL & Mercury, total recoverable \\
\hline HR8 11 & $\mathbf{J}$ & 0.074 & UGL & Mercury, total recoverable \\
\hline HR8 14 & $\mathbf{J}$ & 0.114 & UGL & Mercury, total recoverable \\
\hline HSB 65 & $\mathrm{~J}$ & 0.1555 & UGL & Mercury, total recoverable \\
\hline HSB 65A & $\mathrm{J}$ & 0.121 & UGL & Mercury, total recoverable \\
\hline HSB 68A & $\mathrm{J}$ & 0.1145 & UGL & Mercury, total recoverable \\
\hline HSB 68B & $\mathrm{J}$ & 0.109 & UGL & Mercury, total recoverable \\
\hline HSB 83A & $\mathrm{J}$ & 0.019 & UGL & Mercury, total recoverable \\
\hline HSB 83B & $\mathrm{J}$ & 0.15 & UGL & Mercury, total recoverable \\
\hline HSB 84A & $\mathrm{J}$ & 0.107 & UGL & Mercury, total recoverable \\
\hline HSB 84B & $\mathrm{J}$ & 0.0245 & UGL & Mercury, total recoverable \\
\hline HSB 84C & $\mathrm{J}$ & 0.075 & UGL & Mercury, total recoverable \\
\hline HSB 86A & $J$ & 0.1455 & UGL & Mercury, total recoverable \\
\hline HSB 86B & $\mathrm{J}$ & 0.074 & UGL & Mercury, total recoverable \\
\hline HSB100C & $\mathrm{J}$ & 0.158 & UGL & Mercury, total recoverable \\
\hline HSB 107C & $\mathrm{J}$ & 0.061 & UGL & Mercury, total recoverable \\
\hline HSB110C & $\mathrm{J}$ & 0.024 & UGL & Mercury, total recoverable \\
\hline HSB111C & $\mathrm{J}$ & 0.076 & UGL & Mercury, total recoverable \\
\hline
\end{tabular}




\begin{tabular}{|c|c|c|c|c|}
\hline \multicolumn{5}{|c|}{ Wells $(n=439)$ with median mercury concentration estimated $<0.16 \mathrm{ug} / \mathrm{L}$} \\
\hline well & qualifier & result & units & \begin{tabular}{|r|} 
analyte_name \\
\end{tabular} \\
\hline HSB112C & $\mathrm{J}$ & 0.1565 & UGL & Mercury, total recoverable \\
\hline HSB112E & $\mathrm{J}$ & 0.096 & UGL & Mercury, total recoverable \\
\hline HSB117D & $\mathrm{J}$ & 0.145 & UGL & Mercury, total recoverable \\
\hline HSB 118A & $\mathrm{J}$ & 0.042 & UGL & Mercury, total recoverable \\
\hline HSB 121A & $\mathrm{J}$ & 0.091 & UGL & Mercury, total recoverable \\
\hline HSB 122A & $\mathbf{J}$ & 0.146 & UGL & Mercury, total recoverable \\
\hline HSB 123A & $\mathrm{J}$ & 0.147 & UGL & Mercury, total recoverable \\
\hline $\mathrm{HSB} 125 \mathrm{C}$ & $J$ & 0.0985 & UGL & Mercury, total recoverable \\
\hline HSB129C & $\mathrm{J}$ & 0.155 & UGL & Mercury, total recoverable \\
\hline HSB130D & $\mathbf{J}$ & 0.129 & UGL & Mercury, total recoverable \\
\hline HSB131D & $\mathrm{J}$ & 0.061 & UGL & Mercury, total recoverable \\
\hline HSB133C & $\mathrm{J}$ & 0.1255 & UGL & Mercury, total recoverable \\
\hline HSB133D & $\mathrm{J}$ & 0.118 & UGL & Mercury, total recoverable \\
\hline HSB137D & $\mathrm{J}$ & 0.105 & UGL & Mercury, total recoverable \\
\hline HSB140C & $\mathrm{J}$ & 0.115 & UGL & Mercury, total recoverable \\
\hline HSB 141A & $\mathbf{J}$ & 0.154 & UGL & Mercury, total recoverable \\
\hline HSB141CR & $\mathbf{J}$ & 0.095 & UGL & Mercury, total recoverable \\
\hline HSB141D & $\mathbf{J}$ & 0.092 & UGL & Mercury, total recoverable \\
\hline HSB143C & $\mathbf{J}$ & 0.111 & UGL & Mercury, total recoverable \\
\hline HSB 146A & $\mathbf{J}$ & 0.088 & UGL & Mercury, total recoverable \\
\hline HSB146C & $\mathbf{J}$ & 0.146 & UGL & Mercury, total recoverable \\
\hline HSB149D & $J$ & 0.0775 & UGL & Mercury, total recoverable \\
\hline HSB151C & $\mathbf{J}$ & 0.106 & UGL & Mercury, total recoverable \\
\hline HSB 151D & $\mathrm{J}$ & 0.086 & UGL & Mercury, total recoverable \\
\hline HSB152D & $\mathrm{J}$ & 0.154 & UGL & Mercury, total recoverable \\
\hline HSL 2D & $\mathbf{J}$ & 0.131 & UGL & Mercury, total recoverable \\
\hline HSL 6AA & & 0.1 & UGL & Mercury, total recoverable \\
\hline HSL $6 \mathrm{~B}$ & $\mathbf{J}$ & 0.078 & UGL & Mercury, total recoverable \\
\hline HSL 8A & $\mathrm{J}$ & 0.112 & UGL & Mercury, total recoverable \\
\hline HSL 8B & $\mathrm{J}$ & 0.082 & UGL & Mercury, total recoverable \\
\hline HSL $8 \mathrm{C}$ & $\mathrm{J}$ & 0.0515 & UGL & Mercury, total recoverable \\
\hline KAC 1 & $\mathrm{~J}$ & 0.0905 & UGL & Mercury, total recoverable \\
\hline $\mathrm{KAC} 3$ & $\mathrm{~J}$ & 0.072 & UGL & Mercury, total recoverable \\
\hline $\mathrm{KAC} 4$ & $\mathrm{~J}$ & 0.1205 & UGL & Mercury, total recoverable \\
\hline KAC 5 & $\mathrm{~J}$ & 0.1125 & UGL & Mercury, total recoverable \\
\hline $\mathrm{KAC} 8$ & $\mathbf{J}$ & 0.0845 & UGL & Mercury, total recoverable \\
\hline KAC 9 & $\mathbf{J}$ & 0.08 & UGL & Mercury, total recoverable \\
\hline $\mathrm{KCB} 3$ & $\mathbf{J}$ & 0.108 & UGL & Mercury, total recoverable \\
\hline KCB 5 & $\mathrm{~J}$ & 0.081 & UGL & Mercury, total recoverable \\
\hline KCB 7 & $\mathrm{~J}$ & 0.082 & UGL & Mercury, total recoverable \\
\hline KDB 4 & $\mathrm{~J}$ & 0.019 & UGL & Mercury, total recoverable \\
\hline KSB 5C & $\mathbf{J}$ & 0.137 & UGL & Mercury, total recoverable \\
\hline KSB 5D & $\mathrm{J}$ & 0.143 & UGL & Mercury, total recoverable \\
\hline LAC 1 & J & 0.066 & UGL & Mercury, total recoverable \\
\hline $\mathrm{LAC} 2$ & $\mathbf{J}$ & 0.06 & UGL & Mercury, total recoverable \\
\hline LAC 4 & $\mathbf{J}$ & 0.1175 & UGL & Mercury, total recoverable \\
\hline LAC 5DL & & 0.101 & UGL & Mercury, total recoverable \\
\hline LAC 5DU & & 0.086 & UGL & Mercury, total recoverable \\
\hline LAC 6DU & & 0.154 & UGL & Mercury, total recoverable \\
\hline LAC 8DU & & 0.097 & UGL & Mercury, total recoverable \\
\hline LAW 1E & & 0.113 & UGL & Mercury, total recoverable \\
\hline LAW 2B & $\mathrm{J}$ & 0.1305 & UGL & Mercury, total recoverable \\
\hline LBP 2D & $\mathrm{J}$ & 0.05 & UGL & Mercury, total recoverable \\
\hline
\end{tabular}




\begin{tabular}{|c|c|c|c|c|}
\hline \multicolumn{5}{|c|}{ Wells $(n=439)$ with median mercury concentration estimated $<0.16 \mathrm{ug} / \mathrm{L}$} \\
\hline well & qualifier & result & units & analyte_name \\
\hline LBP 3D & $\mathrm{J}$ & 0.116 & UGL & Mercury, total recoverable \\
\hline $\mathrm{LCO} 2$ & $\mathbf{J}$ & 0.043 & UGL & Mercury, total recoverable \\
\hline LCO 8DU & & 0.117 & UGL & Mercury, total recoverable \\
\hline LFW 8R & $\mathbf{J}$ & 0.043 & UGL & Mercury, total recoverable \\
\hline LFW 10A & $\mathrm{J}$ & 0.103 & UGL & Mercury, total recoverable \\
\hline LFW 18 & $\mathbf{J}$ & 0.063 & UGL & Mercury, total recoverable \\
\hline LFW 22 & $\mathbf{J}$ & 0.085 & UGL & Mercury, total recoverable \\
\hline LFW 23 & $\mathbf{J}$ & 0.031 & UGL & Mercury, total recoverable \\
\hline LFW 23R & $\bar{J}$ & 0.0535 & UGL & Mercury, total recoverable \\
\hline LFW 24 & $J$ & 0.1465 & UGL & Mercury, total recoverable \\
\hline LFW 28 & $\mathrm{~J}$ & 0.148 & UGL & Mercury, total recoverable \\
\hline LFW 29 & $\mathbf{J}$ & 0.134 & UGL & Mercury, total recoverable \\
\hline LFW 32 & $\mathbf{J}$ & 0.047 & UGL & Mercury, total recoverable \\
\hline LFW 36R & $\mathbf{J}$ & 0.08 & UGL & Mercury, total recoverable \\
\hline LFW 43B & $\mathrm{J}$ & 0.0565 & UGL & Mercury, total recoverable \\
\hline LFW 43C & $\mathrm{J}$ & 0.069 & UGL & Mercury, total recoverable \\
\hline LFW 47D & $\mathbf{J}$ & 0.1525 & UGL & Mercury, total recoverable \\
\hline LFW 56D & $\mathrm{J}$ & 0.024 & UGL & Mercury, total recoverable \\
\hline LFW 60C & $\mathbf{J}$ & 0.0205 & UGL & Mercury, total recoverable \\
\hline LFW 63B & $\mathrm{J}$ & 0.055 & UGL & Mercury, total recoverable \\
\hline LFW 63C & $\mathrm{J}$ & 0.069 & UGL & Mercury, total recoverable \\
\hline LFW 63D & $\mathrm{J}$ & 0.15 & $\mathrm{UGL}$ & Mercury, total recoverable \\
\hline LFW 64C & $\mathrm{J}$ & 0.128 & UGL & Mercury, total recoverable \\
\hline LFW 64D & $\mathrm{J}$ & 0.058 & UGL & Mercury, total recoverable \\
\hline LFW 65B & $\mathrm{J}$ & 0.148 & UGL & Mercury, total recoverable \\
\hline LFW 69C & $\mathrm{J}$ & 0.114 & UGL & Mercury, total recoverable \\
\hline LFW 69D & $\mathrm{J}$ & 0.105 & UGL & Mercury, total recoverable \\
\hline LFW 70B & $\mathrm{J}$ & 0.151 & UGL & Mercury, total recoverable \\
\hline LFW 71D & $\bar{J}$ & 0.049 & UGL & Mercury, total recoverable \\
\hline LFW 72B & $\mathrm{J}$ & 0.105 & UGL & Mercury, total recoverable \\
\hline LFW 72C & $\mathrm{J}$ & 0.107 & UGL & Mercury, total recoverable \\
\hline LFW 72D & $\mathrm{J}$ & 0.136 & UGL & Mercury, total recoverable \\
\hline LFW 74C & $\mathrm{J}$ & 0.078 & UGL & Mercury, total recoverable \\
\hline LFW 74D & $\mathrm{J}$ & 0.104 & UGL & Mercury, total recoverable \\
\hline LFW 75C & $\mathrm{J}$ & 0.115 & UGL & Mercury, total recoverable \\
\hline LFW 75D & $\mathrm{J}$ & 0.036 & UGL & Mercury, total recoverable \\
\hline LRP 4 & $\mathbf{J}$ & 0.121 & UGL & Mercury, total recoverable \\
\hline $\mathrm{MCB} 5$ & $\mathbf{J}$ & 0.068 & $\mathrm{UGL}$ & Mercury, total recoverable \\
\hline $\mathrm{MCB} 5 \mathrm{C}$ & $\mathrm{J}$ & 0.0995 & UGL & Mercury, total recoverable \\
\hline $\mathrm{MCB} 6 \mathrm{C}$ & $J$ & 0.05 & UGL & Mercury, total recoverable \\
\hline MCB 10DR & $J$ & 0.032 & UGL & Mercury, total recoverable \\
\hline $\mathrm{MCB} 11 \mathrm{~B}$ & $\mathrm{~J}$ & 0.029 & $\mathrm{UGL}$ & Mercury, total recoverable \\
\hline MCB 11C & $\mathrm{J}$ & 0.1 & UGL & Mercury, total recoverable \\
\hline MCB 12B & $\mathrm{J}$ & 0.044 & UGL & Mercury, total recoverable \\
\hline MCB 12C & $\mathrm{J}$ & 0.064 & UGL & Mercury, total recoverable \\
\hline MCB 13D & $\mathrm{J}$ & 0.047 & UGL & Mercury, total recoverable \\
\hline MCB 14B & $\mathrm{J}$ & 0.0495 & $\mathrm{UGL}$ & Mercury, total recoverable \\
\hline $\mathrm{MCB} 20 \mathrm{~B}$ & $\mathrm{~J}$ & 0.133 & UGL & Mercury, total recoverable \\
\hline MSB $1 \mathrm{CC}$ & $J$ & 0.049 & UGL & Mercury, total recoverable \\
\hline MSB 2B & $\mathrm{J}$ & 0.1395 & UGL & Mercury, total recoverable \\
\hline MSB 3B & $\mathrm{J}$ & 0.04 & UGL & Mercury, total recoverable \\
\hline MSB 4B & $\mathrm{J}$ & 0.046 & UGL & Mercury, total recoverable \\
\hline MSB 4D & $J$ & 0.1 & UGL & Mercury, total recoverable \\
\hline
\end{tabular}




\begin{tabular}{|c|c|c|c|c|}
\hline \multicolumn{5}{|c|}{ Wells $(n=439)$ with median mercury concentration estimated $<0.16 \mathrm{ug} / \mathrm{L}$} \\
\hline well & qualifier & result & units & analyte_name \\
\hline MSB 5A & $\mathbf{J}$ & 0.026 & UGL & Mercury, total recoverable \\
\hline MSB 7A & $\mathrm{J}$ & 0.094 & UGL & Mercury, total recoverable \\
\hline MSB 8A & $\mathbf{J}$ & 0.051 & UGL & Mercury, total recoverable \\
\hline MSB 8B & $\mathrm{J}$ & 0.024 & UGL & Mercury, total recoverable \\
\hline MSB 9C & $\mathrm{J}$ & 0.019 & UGL & Mercury, total recoverable \\
\hline MSB 10B & $\mathrm{J}$ & 0.025 & UGL & Mercury, total recoverable \\
\hline MSB 12B & $\mathrm{J}$ & 0.1555 & UGL & Mercury, total recoverable \\
\hline MSB 13CC & $\mathrm{J}$ & 0.141 & UGL & Mercury, total recoverable \\
\hline MSB 15A & $\mathrm{J}$ & 0.056 & UGL & Mercury, total recoverable \\
\hline MSB 15D & $\mathrm{J}$ & 0.143 & UGL & Mercury, total recoverable \\
\hline MSB 19C & $\mathbf{J}$ & 0.075 & UGL & Mercury, total recoverable \\
\hline MSB 20A & $\mathbf{J}$ & 0.018 & UGL & Mercury, total recoverable \\
\hline MSB 23TA & $\mathrm{J}$ & 0.015 & UGL & Mercury, total recoverable \\
\hline MSB 25A & $\mathrm{J}$ & 0.11 & UGL & Mercury, total recoverable \\
\hline MSB 26 & $\mathrm{~J}$ & 0.029 & UGL & Mercury, total recoverable \\
\hline MSB 26B & $\mathrm{J}$ & 0.089 & UGL & Mercury, total recoverable \\
\hline MSB 27 & $\mathrm{~J}$ & 0.057 & UGL & Mercury, total recoverable \\
\hline MSB 27TA & $\mathrm{J}$ & 0.11 & $\mathrm{UGL}$ & Mercury, total recoverable \\
\hline MSB 28A & $\mathrm{J}$ & 0.0425 & UGL & Mercury, total recoverable \\
\hline MSB 29B & $\mathrm{J}$ & 0.022 & UGL & Mercury, total recoverable \\
\hline MSB 29C & $\mathbf{J}$ & 0.032 & UGL. & Mercury, total recoverable \\
\hline MSB 29D & $\mathbf{J}$ & 0.022 & UGL & Mercury, total recoverable \\
\hline MSB 30C & $\mathbf{J}$ & 0.03 & UGL & Mercury, total recoverable \\
\hline MSB 31B & $\mathbf{J}$ & 0.122 & UGL & Mercury, total recoverable \\
\hline MSB 31C & $\mathbf{J}$ & 0.1495 & UGL & Mercury, total recoverable \\
\hline MSB 31CC & $\mathbf{J}$ & 0.03 & UGL & Mercury, total recoverable \\
\hline MSB 32B & $\mathbf{J}$ & 0.0505 & UGL & Mercury, total recoverable \\
\hline MSB 33C & $\mathrm{J}$ & 0.135 & UGL & Mercury, total recoverable \\
\hline MSB 34B & $\mathbf{J}$ & 0.156 & UGL & Mercury, total recoverable \\
\hline MSB 34C & $\mathrm{J}$ & 0.046 & UGL & Mercury, total recoverable \\
\hline MSB 34TA & $\mathrm{J}$ & 0.033 & UGL & Mercury, total recoverable \\
\hline MSB 36A & $\mathbf{J}$ & 0.0915 & UGL & Mercury, total recoverable \\
\hline MSB 36B & $\mathbf{J}$ & 0.08 & UGL & Mercury, total recoverable \\
\hline MSB 36C & $\mathrm{J}$ & 0.076 & UGL & Mercury, total recoverable \\
\hline MSB 36D & $\mathbf{J}$ & 0.121 & UGL & Mercury, total recoverable \\
\hline MSB 36TA & $\mathbf{J}$ & 0.051 & UGL & Mercury, total recoverable \\
\hline MSB 37B & $\mathrm{J}$ & 0.142 & UGL & Mercury, total recoverable \\
\hline MSB 37C & $\mathrm{J}$ & 0.066 & UGL & Mercury, total recoverable \\
\hline MSB 37TA & $\mathrm{J}$ & 0.023 & UGL & Mercury, total recoverable \\
\hline MSB 38C & $\mathrm{J}$ & 0.103 & $\mathrm{UGL}$ & Mercury, total recoverable \\
\hline MSB 40B & $J$ & 0.054 & UGL & Mercury, total recoverable \\
\hline MSB 41B & $\mathbf{J}$ & 0.018 & UGL & Mercury, total recoverable \\
\hline MSB 41TA & $\mathrm{J}$ & 0.052 & $\mathrm{UGL}$ & Mercury, total recoverable \\
\hline MSB 43B & $\mathrm{J}$ & 0.031 & UGL & Mercury, total recoverable \\
\hline MSB 43D & $\mathbf{J}$ & 0.02 & UGL & Mercury, total recoverable \\
\hline MSB 43TA & $\mathbf{J}$ & 0.082 & UGL & Mercury, total recoverable \\
\hline MSB 45A & $\mathbf{J}$ & 0.056 & UGL & Mercury, total recoverable \\
\hline MSB 45B & $\mathbf{J}$ & 0.057 & UGL & Mercury, total recoverable \\
\hline MSB 46A & $\mathbf{J}$ & 0.018 & UGL & Mercury, total recoverable \\
\hline MSB 47B & $\mathbf{J}$ & 0.156 & UGL & Mercury, total recoverable \\
\hline MSB 47C & $\mathrm{J}$ & 0.085 & UGL & Mercury, total recoverable \\
\hline MSB 47TA & $\mathrm{J}$ & 0.029 & UGL & Mercury, total recoverable \\
\hline MSB 48A & $\mathbf{J}$ & 0.039 & UGL & Mercury, total recoverable \\
\hline
\end{tabular}




\begin{tabular}{|c|c|c|c|c|}
\hline \multicolumn{5}{|c|}{ Wells $(n=439)$ with median mercury concentration estimated $<0.16 \mathrm{ug} / \mathrm{L}$} \\
\hline well & qualifier & result & units & analyte_name \\
\hline MSB 48C & $\mathrm{J}$ & 0.105 & UGL & Mercury, total recoverable \\
\hline MSB 49B & $\mathbf{J}$ & 0.095 & UGL & Mercury, total recoverable \\
\hline MSB 49D & $\mathbf{J}$ & 0.026 & UGL & Mercury, total recoverable \\
\hline MSB 51B & $\mathrm{J}$ & 0.022 & UGL & Mercury, total recoverable \\
\hline MSB 55C & $\mathbf{J}$ & 0.1445 & UGL & Mercury, total recoverable \\
\hline MSB 55D & $\mathrm{J}$ & 0.043 & UGL & Mercury, total recoverable \\
\hline MSB 56D & $\mathrm{J}$ & 0.064 & UGL & Mercury, total recoverable \\
\hline MSB 57D & $\mathrm{J}$ & 0.042 & UGL & Mercury, total recoverable \\
\hline MSB 58D & $\mathrm{J}$ & 0.082 & UGL & Mercury, total recoverable \\
\hline MSB 60D & $\mathrm{J}$ & 0.0375 & UGL & Mercury, total recoverable \\
\hline MSB 62B & $J$ & 0.016 & UGL & Mercury, total recoverable \\
\hline MSB 62D & $\mathrm{J}$ & 0.1575 & UGL & Mercury, total recoverable \\
\hline MSB 63C & $\mathrm{J}$ & 0.027 & UGL & Mercury, total recoverable \\
\hline MSB 63D & $\mathrm{J}$ & 0.045 & UGL & Mercury, total recoverable \\
\hline MSB 64D & $\mathrm{J}$ & 0.126 & UGL & Mercury, total recoverable \\
\hline MSB 65D & $\mathrm{J}$ & 0.119 & UGL & Mercury, total recoverable \\
\hline MSB 66C & $\overline{\mathrm{J}}$ & 0.022 & UGL & Mercury, total recoverable \\
\hline MSB 68B & $\mathrm{J}$ & 0.021 & UGL & Mercury, total recoverable \\
\hline MSB 72B & $\mathbf{J}$ & 0.118 & $\mathrm{UGL}$ & Mercury, total recoverable \\
\hline MSB 76C & $\mathrm{J}$ & 0.091 & UGL & Mercury, total recoverable \\
\hline MSB 82A & $\mathbf{J}$ & 0.044 & UGL & Mercury, total recoverable \\
\hline MSB 82B & $\mathbf{J}$ & 0.0915 & UGL & Mercury, total recoverable \\
\hline MSB 86C & $\mathbf{J}$ & 0.1405 & UGL & Mercury, total recoverable \\
\hline MSB 88B & $\mathrm{J}$ & 0.033 & UGL & Mercury, total recoverable \\
\hline MSB 88C & $\mathbf{J}$ & 0.025 & UGL & Mercury, total recoverable \\
\hline NBG 5 & $\mathbf{J}$ & 0.141 & UGL & Mercury, total recoverable \\
\hline $\mathrm{P} 26 \mathrm{~A}$ & $\mathrm{~J}$ & 0.0495 & UGL & Mercury, total recoverable \\
\hline $\mathrm{P} 26 \mathrm{D}$ & $\mathrm{J}$ & 0.058 & UGL & Mercury, total recoverable \\
\hline PAC 1 & $\mathrm{~J}$ & 0.081 & UGL & Mercury, total recoverable \\
\hline PAC 4 & $\mathrm{~J}$ & 0.041 & UGL & Mercury, total recoverable \\
\hline PBP 2D & $\mathrm{J}$ & 0.05 & UGL & Mercury, total recoverable \\
\hline PCB 4A & $\mathrm{J}$ & 0.13 & UGL & Mercury, total recoverable \\
\hline PRP 2. & $\mathrm{J}$ & 0.07 & UGL & Mercury, total recoverable \\
\hline PRP 3 & $\mathrm{~J}$ & 0.08 & UGL & Mercury, total recoverable \\
\hline RAC 1 & $\mathrm{J}$ & 0.11 & UGL & Mercury, total recoverable \\
\hline RBP 1D & $\mathrm{J}$ & 0.07 & UGL & Mercury, total recoverable \\
\hline RBP 2D & $\mathrm{J}$ & 0.101 & UGL & Mercury, total recoverable \\
\hline RBP 3D & $\mathrm{J}$ & 0.11 & UGL & Mercury, total recoverable \\
\hline RWM 3 & $\mathrm{~J}$ & 0.107 & UGL & Mercury, total recoverable \\
\hline RWM 6 & $\mathrm{~J}$ & 0.045 & UGL & Mercury, total recoverable \\
\hline RWM 8 & $\mathrm{~J}$ & 0.052 & UGL & Mercury, total recoverable \\
\hline RWM 9 & $\mathrm{~J}$ & 0.139 & UGL & Mercury, total recoverable \\
\hline RWM 10 & $\mathrm{~J}$ & 0.11 & UGL & Mercury, total recoverable \\
\hline RWM 11 & $\mathbf{J}$ & 0.083 & $\mathrm{UGL}$ & Mercury, total recoverable \\
\hline SRW 1 & $\mathbf{J}$ & 0.054 & UGL & Mercury, total recoverable \\
\hline SRW 2A & $\mathrm{J}$ & 0.059 & UGL & Mercury, total recoverable \\
\hline SRW 2B & $\mathbf{J}$ & 0.0415 & UGL & Mercury, total recoverable \\
\hline SRW 4BB & $\mathbf{J}$ & 0.054 & UGL & Mercury, total recoverable \\
\hline SRW 5 & $\mathbf{J}$ & 0.142 & UGL & Mercury, total recoverable \\
\hline SRW 10 & $\mathbf{J}$ & 0.044 & UGL & Mercury, total recoverable \\
\hline SRW 14A & $\mathbf{J}$ & 0.066 & UGL & Mercury, total recoverable \\
\hline SRW 15A & $\mathbf{J}$ & 0.05 & UGL & Mercury, total recoverable \\
\hline SRW 16B & $\mathrm{J}$ & 0.0885 & UGL & Mercury, total recoverable \\
\hline
\end{tabular}




\begin{tabular}{l|c|c|c|l}
\hline \multicolumn{5}{c|}{ Wells $(n=439)$ with median mercury concentration estimated $<0.16 \mathrm{ug} / \mathrm{L}$} \\
\hline well & qualifier & result & units & \multicolumn{1}{|c}{ analyte name } \\
\hline SSS 25 & J & 0.084 & UGL & Mercury, total recoverable \\
\hline STR312I & J & 0.158 & UGL & Mercury, total recoverable \\
\hline TBG 1 & J & 0.061 & UGL & Mercury, total recoverable \\
\hline TBG 5A & J & 0.09 & UGL & Mercury, total recoverable \\
\hline TBG 5B & J & 0.086 & UGL & Mercury, total recoverable \\
\hline TNX 11D & J & 0.048 & UGL & Mercury, total recoverable \\
\hline TNX 26D & J & 0.12 & UGL & Mercury, total recoverable \\
\hline TRW 3 & J & 0.08 & UGL & Mercury, total recoverable \\
\hline TRW 4 & J & 0.09 & UGL & Mercury, total recoverable \\
\hline XSB 1D & J & 0.103 & UGL & Mercury, total recoverable \\
\hline YSB 1A & J & 0.09 & UGL & Mercury, total recoverable \\
\hline YSB 2A & J & 0.038 & UGL & Mercury, total recoverable \\
\hline YSB 4A & J & 0.098 & UGL & Mercury, total recoverable \\
\hline ZBG 1 & J & 0.112 & UGL & Mercury, total recoverable \\
\hline ZBG 2 & J & 0.116 & UGL & Mercury, total recoverable \\
\hline
\end{tabular}


Appendix B: Estimated Median Concentrations for SRS Groundwater Wells for "Undetermined" Population.

(total mercury between $0.16 \mathrm{ug} / \mathrm{L}$ and $0.4 \mathrm{ug} / \mathrm{L}$ ) 
Wells ( $\mathrm{n}=272)$ with median mercury concentration estimated between $0.16 \& 0.4 \mathrm{ug} / \mathrm{L}$

\begin{tabular}{|c|c|c|c|c|}
\hline well & qualifier & result & units & analyte_name \\
\hline$A C \quad 3 A$ & $\mathrm{~J}$ & 0.175 & UGL & Mercury, total recoverable \\
\hline $\mathrm{ACB} 3 \mathrm{~A}$ & & 0.26 & UGL & Mercury, total recoverable \\
\hline ACB 4A & $\mathbf{J}$ & 0.29714286 & $\overline{U G L}$ & Mercury, total recoverable \\
\hline AMB 11D & & 0.237 & $\overline{\mathrm{UGL}}$ & Mercury, total recoverable \\
\hline AMB 13AR & $\mathbf{J}$ & 0.161 & UGL & Mercury, total recoverable \\
\hline AMB $19 \mathrm{C}$ & $\mathbf{J}$ & 0.172 & UGL & Mercury, total recoverable \\
\hline ARP 1A & & 0.2205 & UGL & Mercury, total recoverable \\
\hline ARP 5D & & 0.24 & $\overline{\mathrm{UGL}}$ & Mercury, total recoverable \\
\hline ASB 2CR & & 0.232 & UGL & Mercury, total recoverable \\
\hline BG 52 & & 0.288 & UGL & Mercury, total recoverable \\
\hline BG 54 & & 0.246 & UGL & Mercury, total recoverable \\
\hline BG 121 & $\mathbf{J}$ & 0.204 & UGL & Mercury, total recoverable \\
\hline BGO 7D & $\mathrm{J}$ & 0.192 & UGL & Mercury, total recoverable \\
\hline BGO 16AR & $\mathbf{J}$ & 0.205 & UGL & Mercury, total recoverable \\
\hline BGO 28D & $\mathrm{J}$ & 0.1715 & UGL & Mercury, total recoverable \\
\hline BGO 30D & $\mathrm{J}$ & 0.1725 & UGL & Mercury, total recoverable \\
\hline BGO 31D & & 0.258 & UGL & Mercury, total recoverable \\
\hline BGO 44A & $\mathbf{J}$ & 0.235 & UGL & Mercury, total recoverable \\
\hline BGO 47A & $\mathbf{J}$ & 0.19 & UGL & Mercury, total recoverable \\
\hline BGO $52 \mathrm{C}$ & $\mathbf{J}$ & 0.189 & UGL & Mercury, total recoverable \\
\hline BGX 4A & $\mathrm{J}$ & 0.164 & $\overline{\text { UGL }}$ & Mercury, total recoverable \\
\hline CMP 11D & $\mathbf{J}$ & 0.164 & UGL & Mercury, total recoverable \\
\hline CRP 5C & & 0.22 & $\overline{\text { UGL }}$ & Mercury, total recoverable \\
\hline CRP 7D & $\mathrm{J}$ & 0.166 & UGL & Mercury, total recoverable \\
\hline CRP 8D & & 0.207 & $\overline{\text { UGL }}$ & Mercury, total recoverable \\
\hline CSB 6A & $J$ & 0.276 & UGL & Mercury, total recoverable \\
\hline CSR 5D & & 0.212 & UGL & Mercury, total recoverable \\
\hline CSR 6D & $\mathrm{J}$ & 0.1805 & UGL & Mercury, total recoverable \\
\hline DCB 1A & & 0.3 & UGL & Mercury, total recoverable \\
\hline DCB 7 & & 0.2045 & UGL & Mercury, total recoverable \\
\hline DCB 10 & & 0.206 & UGL & Mercury, total recoverable \\
\hline DCB 15 & & 0.218 & UGL & Mercury, total recoverable \\
\hline DCB 21A & $\mathbf{J}$ & 0.207 & UGL & Mercury, total recoverable \\
\hline$\overline{\mathrm{DCB} 22 \mathrm{~B}}$ & $\mathbf{J}$ & 0.194 & UGL & Mercury, total recoverable \\
\hline$\overline{\mathrm{DCB} 24 \mathrm{~A}}$ & $\mathrm{~J}$ & 0.16 & UGL & Mercury, total recoverable \\
\hline$\overline{D C B} 24 B$ & $\mathrm{~J}$ & 0.2 & $\overline{\text { UGL }}$ & Mercury, total recoverable \\
\hline DOB 7 & & 0.224 & UGL & Mercury, total recoverable \\
\hline DOB 10 & & 0.215 & UGL & Mercury, total recoverable \\
\hline $\begin{array}{ll}F \quad 15 \\
\end{array}$ & $\mathrm{~J}$ & 0.255 & UGL & Mercury, total recoverable \\
\hline $\begin{array}{ll}\mathrm{F} 17 \\
\end{array}$ & $\mathrm{~J}$ & 0.393 & UGL & Mercury, total recoverable \\
\hline F 25 & & 0.23 & $\overline{\text { UGL }}$ & Mercury, total recoverable \\
\hline FAB I & & 0.3285 & UGL & Mercury, total recoverable \\
\hline FAB 4 & $\mathbf{J}$ & 0.1675 & UGL & Mercury, total recoverable \\
\hline FAC 3 & & 0.378 & UGL & Mercury, total recoverable \\
\hline$\overline{\text { FAC } 4}$ & & 0.367 & $\overline{U G L}$ & Mercury, total recoverable \\
\hline FAC 5 & $\mathbf{J}$ & 0.189 & UGL & Mercury, total recoverable \\
\hline FAC 6 & & 0.3055 & UGL & Mercury, total recoverable \\
\hline FAC 7 & & 0.385 & UGL & Mercury, total recoverable \\
\hline FAC 8 & & 0.2955 & UGL & Mercury, total recoverable \\
\hline FBP 2A & & 0.207 & UGL & Mercury, total recoverable \\
\hline FCA 16A & & 0.207 & UGL & Mercury, total recoverable \\
\hline FEX 1TK & & 0.301 & UGL & Mercury, total recoverable \\
\hline FNB 1 & $\mathbf{J}$ & 0.179 & UGL & Mercury, total recoverable \\
\hline
\end{tabular}




\begin{tabular}{|c|c|c|c|c|}
\hline \multicolumn{5}{|c|}{ Wells $(n=272)$ with median mercury concentration estimated between $0.16 \& 0.4 \mathrm{ug} / \mathrm{L}$} \\
\hline well & qualifier & result & units & analyte_name \\
\hline FNB 3 & & 0.223 & UGL & Mercury, total recoverable \\
\hline FNB 5 & & 0.223 & UGL & Mercury, total recoverable \\
\hline FNB 5 & & 0.225 & UGL & Mercury, total recoverable \\
\hline FSB 87D & $\mathrm{J}$ & 0.219 & UGL & Mercury, total recoverable \\
\hline FSB 91D & $\mathbf{J}$ & 0.211 & UGL & Mercury, total recoverable \\
\hline FSB 93C & $\mathrm{J}$ & 0.201 & UGL & Mercury, total recoverable \\
\hline FSB 93D & & 0.204 & UGL & Mercury, total recoverable \\
\hline FSB 104D & $\mathrm{J}$ & 0.21 & UGL & Mercury, total recoverable \\
\hline FSB $105 C$ & & 0.249 & UGL & Mercury, total recoverable \\
\hline FSB112C & & 0.378 & UGL & Mercury, total recoverable \\
\hline FSB 112D & & 0.202 & UGL & Mercury, total recoverable \\
\hline FSB 118D & $\mathrm{J}$ & 0.1835 & UGL & Mercury, total recoverable \\
\hline FSB121C & $\mathrm{J}$ & 0.1965 & UGL & Mercury, total recoverable \\
\hline FSL 6D & & 0.227 & UGL & Mercury, total recoverable \\
\hline FSL 7D & $\mathrm{J}$ & 0.183 & UGL & Mercury, total recoverable \\
\hline FSS 1D & $\mathrm{J}$ & 0.227 & UGL & Mercury, total recoverable \\
\hline FSS 2D & $\mathrm{J}$ & 0.2365 & $\overline{\mathrm{UGL}}$ & Mercury, total recoverable \\
\hline FSS 4D & $\mathrm{J}$ & 0.201 & UGL & Mercury, total recoverable \\
\hline FTF 1 & & 0.21 & UGL & Mercury, total recoverable \\
\hline FTF 4 & & 0.315 & $\overline{\text { UGL }}$ & Mercury, total recoverable \\
\hline FTF 4 & & 0.37 & UGL & Mercury, total recoverable \\
\hline FTF 10 & $\mathrm{~J}$ & 0.2545 & UGL & Mercury, total recoverable \\
\hline FTF 16 & & 0.23 & UGL & Mercury, total recoverable \\
\hline FTF 17 & $\mathrm{~J}$ & 0.2155 & UGL & Mercury, total recoverable \\
\hline FTF 18 & $\mathrm{~J}$ & 0.222 & UGL & Mercury, total recoverable \\
\hline FTF 23 & & 0.2 & UGL & Mercury, total recoverable \\
\hline FTF 24A & & 0.25 & UGL & Mercury, total recoverable \\
\hline FTF 26 & & 0.342 & UGL & Mercury, total recoverable \\
\hline HAA 3D & $\mathbf{J}$ & 0.1755 & UGL & Mercury, total recoverable \\
\hline HAA 4D & & 0.16 & UGL & Mercury, total recoverable \\
\hline HAA $5 \mathrm{C}$ & & 0.359 & UGL & Mercury, total recoverable \\
\hline HAC 3 & & 0.3225 & UGL & Mercury, total recoverable \\
\hline $\mathrm{HAC} 4$ & $\mathrm{~J}$ & 0.1855 & UGL & Mercury, total recoverable \\
\hline $\mathrm{HC} \quad 4 \mathrm{~B}$ & $\mathrm{~J}$ & 0.204 & UGL & Mercury, total recoverable \\
\hline HET 3D & $\mathrm{J}$ & 0.304 & UGL & Mercury, total recoverable \\
\hline HET 4D & & 0.2 & UGL & Mercury, total recoverable \\
\hline HR3 11 & & 0.225 & UGL & Mercury, total recoverable \\
\hline HR3 15DU & & 0.264 & UGL & Mercury, total recoverable \\
\hline HSB 65B & $\mathrm{J}$ & 0.1925 & UGL & Mercury, total recoverable \\
\hline HSB $65 \mathrm{C}$ & $\mathbf{J}$ & 0.1875 & UGL & Mercury, total recoverable \\
\hline HSB 66 & $J$ & 0.2295 & UGL & Mercury, total recoverable \\
\hline HSB 69 & $\mathbf{J}$ & 0.199 & UGL & Mercury, total recoverable \\
\hline HSB 70 & $\mathbf{J}$ & 0.2 & UGL & Mercury, total recoverable \\
\hline HSB 70C & $\mathbf{J}$ & 0.1725 & UGL & Mercury, total recoverable \\
\hline HSB 71 & $\mathbf{J}$ & 0.192 & UGL & Mercury, total recoverable \\
\hline HSB 71C & & 0.2055 & UGL & Mercury, total recoverable \\
\hline HSB $83 \mathrm{C}$ & $\mathbf{J}$ & 0.2 & UGL & Mercury, total recoverable \\
\hline HSB 83D & J & 0.32 & UGL & Mercury, total recoverable \\
\hline HSB 84D & J & 0.187 & UGL & Mercury, total recoverable \\
\hline HSB 85A & $\mathbf{J}$ & 0.2 & UGL & Mercury, total recoverable \\
\hline HSB $85 \mathrm{C}$ & & 0.2 & UGL & Mercury, total recoverable \\
\hline HSB 86C & & 0.368 & UGL & Mercury, total recoverable \\
\hline HSB 86D & J & 0.263 & UGL & Mercury, total recoverable \\
\hline
\end{tabular}




\begin{tabular}{|c|c|c|c|c|}
\hline \multicolumn{5}{|c|}{ Wells $(n=272$ ) with median mercury concentration estimated between $0.16 \& 0.4 \mathrm{ug} / \mathrm{L}$} \\
\hline well & qualifier & result & units & analyte_name \\
\hline HSB100D & $\mathrm{J}$ & 0.2165 & UGL & Mercury, total recoverable \\
\hline HSB105C & $\mathbf{J}$ & 0.2 & UGL & Mercury, total recoverable \\
\hline HSB109C & & 0.2 & UGL & Mercury, total recoverable \\
\hline HSB111D & $\mathbf{J}$ & 0.2 & UGL & Mercury, total recoverable \\
\hline HSB111E & $\mathbf{J}$ & 0.2 & UGL & Mercury, total recoverable \\
\hline HSB 112D & $\mathrm{J}$ & 0.2 & UGL & Mercury, total recoverable \\
\hline HSB113C & $\mathbf{J}$ & 0.2 & UGL & Mercury, total recoverable \\
\hline HSB113D & & 0.398 & UGL & Mercury, total recoverable \\
\hline HSB114C & & 0.239 & UGL & Mercury, total recoverable \\
\hline HSB115C & $\mathrm{J}$ & 0.186 & UGL & Mercury, total recoverable \\
\hline HSB115D & $J$ & 0.2 & UGL & Mercury, total recoverable \\
\hline HSB116D & $\mathrm{J}$ & 0.2 & UGL & Mercury, total recoverable \\
\hline HSB 117A & $\mathrm{J}$ & 0.2 & UGL & Mercury, total recoverable \\
\hline HSB117C & $\mathrm{J}$ & 0.2385 & UGL & Mercury, total recoverable \\
\hline HSB119A & $\mathrm{J}$ & 0.2 & UGL & Mercury, total recoverable \\
\hline HSB 120A & $\mathbf{J}$ & 0.202 & UGL & Mercury, total recoverable \\
\hline HSB 124AR & $\mathrm{J}$ & 0.1745 & UGL & Mercury, total recoverable \\
\hline HSB126C & $\mathbf{J}$ & 0.2 & UGL & Mercury, total recoverable \\
\hline HSB127C & $\mathbf{J}$ & 0.2 & UGL & Mercury, total recoverable \\
\hline HSB129D & & 0.323 & UGL & Mercury, total recoverable \\
\hline $\mathrm{HSB} 130 \mathrm{C}$ & $\mathrm{J}$ & 0.1665 & UGL & Mercury, total recoverable \\
\hline HSB131C & $\mathrm{J}$ & 0.2 & $\mathrm{UGL}$ & Mercury, total recoverable \\
\hline HSB134C & $J$ & 0.202 & UGL & Mercury, total recoverable \\
\hline HSB135D & & 0.225 & UGL & Mercury, total recoverable \\
\hline HSB136C & $\mathrm{J}$ & 0.2 & UGL & Mercury, total recoverable \\
\hline HSB136D & $\mathbf{J}$ & 0.217 & UGL & Mercury, total recoverable \\
\hline HSB137C & $\mathrm{J}$ & 0.2 & UGL & Mercury, total recoverable \\
\hline HSB138D & $\mathbf{J}$ & 0.179 & UGL & Mercury, total recoverable \\
\hline HSB140D & $J$ & 0.1745 & UGL & Mercury, total recoverable \\
\hline HSB143D & $\mathbf{J}$ & 0.188 & $\mathrm{UGL}$ & Mercury, total recoverable \\
\hline HSB144A & $\mathbf{J}$ & 0.1915 & UGL & Mercury, total recoverable \\
\hline HSB145C & & 0.348 & UGL & Mercury, total recoverable \\
\hline HSB148C & $\mathbf{J}$ & 0.173 & UGL & Mercury, total recoverable \\
\hline HSB150D & $\mathrm{J}$ & 0.2 & UGL & Mercury, total recoverable \\
\hline HSB152C & $\mathrm{J}$ & 0.1945 & UGL & Mercury, total recoverable \\
\hline HSL 1D & $\mathrm{J}$ & 0.29 & UGL & Mercury, total recoverable \\
\hline HSL 3D & $\mathbf{J}$ & 0.1805 & UGL & Mercury, total recoverable \\
\hline HSL 4D & $\mathbf{J}$ & 0.193 & UGL & Mercury, total recoverable \\
\hline HSL 5D & $\mathbf{J}$ & 0.191 & UGL & Mercury, total recoverable \\
\hline HSL 6D & & 0.229 & UGL & Mercury, total recoverable \\
\hline HSL 7D & $\mathbf{J}$ & 0.2 & UGL & Mercury, total recoverable \\
\hline HSL 8D & $\mathrm{J}$ & 0.2 & UGL & Mercury, total recoverable \\
\hline HTF 1 & $J$ & 0.189 & UGL & Mercury, total recoverable \\
\hline HTF 6 & & 0.286 & UGL & Mercury, total recoverable \\
\hline HTF 7 & $\mathrm{~J}$ & 0.1865 & UGL & Mercury, total recoverable \\
\hline HTF 9 & & 0.337 & UGL & Mercury, total recoverable \\
\hline HTF 10 & & 0.22 & UGL & Mercury, total recoverable \\
\hline HTF 11 & $\mathbf{J}$ & 0.321 & UGL & Mercury, total recoverable \\
\hline HTF 12 & & 0.336 & UGL & Mercury, total recoverable \\
\hline HTF 14 & & 0.2025 & UGL & Mercury, total recoverable \\
\hline HTF 18 & & 0.34 & UGL & Mercury, total recoverable \\
\hline HTF 19 & $\mathrm{~J}$ & 0.2 & UGL & Mercury, total recoverable \\
\hline HTF 23 & & 0.3685 & UGL & Mercury, total recoverable \\
\hline
\end{tabular}




\begin{tabular}{|c|c|c|c|c|}
\hline \multicolumn{5}{|c|}{ Wells ( $n=272$ ) with median mercury concentration estimated between $0.16 \& 0.4 \mathrm{ug} / \mathrm{L}$} \\
\hline well & qualifier & result & units & analyte_name \\
\hline HTF 25 & & 0.2295 & UGL & Mercury, total recoverable \\
\hline HTF 27 & $J$ & 0.318 & UGL & Mercury, total recoverable \\
\hline HTF 28 & $\mathbf{J}$ & 0.2 & UGL & Mercury, total recoverable \\
\hline HTF 31 & & 0.36 & UGL & Mercury, total recoverable \\
\hline HTF 32 & $\mathrm{~J}$ & 0.23 & $\mathrm{UGL}$ & Mercury, total recoverable \\
\hline HWP 2D & $\mathrm{J}$ & 0.183 & UGL & Mercury, total recoverable \\
\hline HXB 4D & $\mathrm{J}$ & 0.1685 & UGL & Mercury, total recoverable \\
\hline $\mathrm{HXB}$ 6D & $\mathrm{J}$ & 0.173 & UGL & Mercury, total recoverable \\
\hline IDB 2A & & 0.171 & UGL & Mercury, total recoverable \\
\hline KBP 2D & & 0.3255 & UGL & Mercury, total recoverable \\
\hline KCB 6 & $\mathbf{J}$ & 0.18 & UGL & Mercury, total recoverable \\
\hline $\mathrm{KDB} 5$ & & 0.209 & UGL & Mercury, total recoverable \\
\hline LAC 3 & & 0.189 & UGL & Mercury, total recoverable \\
\hline LAC 7DL & & 0.173 & UGL & Mercury, total recoverable \\
\hline LDB 4 & & 0.189 & UGL & Mercury, total recoverable \\
\hline LFW 6R & $\mathbf{J}$ & 0.2 & UGL & Mercury, total recoverable \\
\hline LFW 16 & & 0.299 & UGL & Mercury, total recoverable \\
\hline LFW 21 & $\mathbf{J}$ & 0.2 & UGL & Mercury, total recoverable \\
\hline LFW 31 & $\mathbf{J}$ & 0.1605 & UGL & Mercury, total recoverable \\
\hline LFW 38 & & 0.265 & UGL & Mercury, total recoverable \\
\hline LFW 39 & & 0.2 & UGL & Mercury, total recoverable \\
\hline LFW 43D & $\mathbf{J}$ & 0.162 & UGL & Mercury, total recoverable \\
\hline LFW 45D & $\mathrm{J}$ & 0.2 & UGL & Mercury, total recoverable \\
\hline LFW 47C & $J$ & 0.1995 & UGL & Mercury, total recoverable \\
\hline LFW 55C & $\mathrm{J}$ & 0.2 & UGL & Mercury, total recoverable \\
\hline LFW 57D & $\mathbf{J}$ & 0.193 & UGL & Mercury, total recoverable \\
\hline LFW 58D & & 0.249 & UGL & Mercury, total recoverable \\
\hline LFW 59D & $\mathrm{J}$ & 0.2 & UGL & Mercury, total recoverable \\
\hline LFW 60D & $\mathrm{J}$ & 0.195 & UGL & Mercury, total recoverable \\
\hline LFW 61D & $\mathrm{J}$ & 0.2 & UGL & Mercury, total recoverable \\
\hline LFW 65D & $\mathrm{J}$ & 0.166 & UGL & Mercury, total recoverable \\
\hline LFW 67C & $\mathrm{J}$ & 0.291 & UGL & Mercury, total recoverable \\
\hline LFW 67D & $\mathrm{J}$ & 0.19 & UGL & Mercury, total recoverable \\
\hline LFW 68D & & 0.207 & UGL & Mercury, total recoverable \\
\hline LFW 71B & $\mathrm{I}$ & 0.186 & UGL & Mercury, total recoverable \\
\hline MGA 36 & $\mathbf{J}$ & 0.2 & UGL & Mercury, total recoverable \\
\hline MGC 9 & $\mathbf{J}$ & 0.26 & $\mathrm{UGL}$ & Mercury, total recoverable \\
\hline MSB 5C & $\mathrm{J}$ & 0.208 & UGL & Mercury, total recoverable \\
\hline MSB 6B & $\mathbf{J}$ & 0.196 & UGL & Mercury, total recoverable \\
\hline MSB 7C & & 0.216 & UGL & Mercury, total recoverable \\
\hline MSB 8C & & 0.243 & UGL & Mercury, total recoverable \\
\hline MSB 11C & & 0.361 & UGL & Mercury, total recoverable \\
\hline MSB 14A & $\mathrm{J}$ & 0.17 & UGL & Mercury, total recoverable \\
\hline MSB 14B & & 0.263 & UGL & Mercury, total recoverable \\
\hline MSB 15C & $\mathbf{J}$ & 0.1705 & UGL & Mercury, total recoverable \\
\hline MSB 17B & & 0.219 & UGL & Mercury, total recoverable \\
\hline MSB 21A & $\mathbf{J}$ & 0.191 & UGL & Mercury, total recoverable \\
\hline MSB 23B & $\mathrm{J}$ & 0.273 & UGL & Mercury, total recoverable \\
\hline MSB 24 & $\mathbf{J}$ & 0.161 & UGL & Mercury, total recoverable \\
\hline MSB 38B & $\mathrm{J}$ & 0.2 & UGL & Mercury, total recoverable \\
\hline MSB 39C & $\mathbf{J}$ & 0.2 & UGL & Mercury, total recoverable \\
\hline MSB 39D & $\mathrm{J}$ & 0.178 & UGL & Mercury, total recoverable \\
\hline MSB 42B & $\mathbf{J}$ & 0.203 & $\mathrm{UGL}$ & Mercury, total recoverable \\
\hline
\end{tabular}




\begin{tabular}{|c|c|c|c|c|}
\hline \multicolumn{5}{|c|}{ Wells $(n=272)$ with median mercury concentration estimated between $0.16 \& 0.4 \mathrm{ug} / \mathrm{L}$} \\
\hline well & qualifier & result & units & \begin{tabular}{|c|} 
analyte_name \\
\end{tabular} \\
\hline MSB 42C & $\mathrm{J}$ & 0.193 & UGL & Mercury, total recoverable \\
\hline MSB 42D & $\mathrm{J}$ & 0.2 & UGL & Mercury, total recoverable \\
\hline MSB 47D & & 0.372 & UGL & Mercury, total recoverable \\
\hline MSB 48B & & 0.2 & UGL & Mercury, total recoverable \\
\hline MSB 59D & $J$ & 0.169 & UGL & Mercury, total recoverable \\
\hline MSB 62C & & 0.23 & UGL & Mercury, total recoverable \\
\hline MSB 63B & $\mathbf{J}$ & 0.185 & UGL & Mercury, total recoverable \\
\hline MSB 64C & & 0.3385 & UGL & Mercury, total recoverable \\
\hline MSB 66B & $\mathrm{J}$ & 0.191 & UGL & Mercury, total recoverable \\
\hline MSB 67C & $\mathrm{J}$ & 0.298 & UGL & Mercury, total recoverable \\
\hline MSB 82C & $\mathrm{J}$ & 0.16 & UGL & Mercury, total recoverable \\
\hline MSB 84A & $\mathrm{J}$ & 0.172 & UGL & Mercury, total recoverable \\
\hline PCB 2A & & 0.335 & UGL & Mercury, total recoverable \\
\hline PRP 4 & & 0.265 & UGL & Mercury, total recoverable \\
\hline$\overline{R A C ~} 4$ & & 0.2895 & UGL & Mercury, total recoverable \\
\hline RSA 9 & $J$ & 0.185 & UGL & Mercury, total recoverable \\
\hline RSA 10 & & 0.2345 & UGL & Mercury, total recoverable \\
\hline RSB 8 & & 0.306 & UGL & Mercury, total recoverable \\
\hline RSB 9 & & 0.2 & $\overline{\mathrm{UGL}}$ & Mercury, total recoverable \\
\hline RSC 3 & & 0.311 & UGL & Mercury, total recoverable \\
\hline RSC 4 & & 0.234 & UGL & Mercury, total recoverable \\
\hline RSC 6 & & 0.214 & UGL & Mercury, total recoverable \\
\hline RSC 7 & & 0.37 & UGL & Mercury, total recoverable \\
\hline RSC 8 & & 0.2 & UGL & Mercury, total recoverable \\
\hline RSC 9 & & 0.2 & $\overline{\text { UGL }}$ & Mercury, total recoverable \\
\hline RSD 4 & & 0.2235 & UGL & Mercury, total recoverable \\
\hline RSD 8 & & 0.205 & UGL & Mercury, total recoverable \\
\hline RSD 10 & & 0.2425 & UGL & Mercury, total recoverable \\
\hline RSE 4B & & 0.34 & UGL & Mercury, total recoverable \\
\hline RSE 4C & & 0.3585 & UGL & Mercury, total recoverable \\
\hline RWM 2 & $J$ & 0.172 & UGL & Mercury, total recoverable \\
\hline RWM 5 & $J$ & 0.175 & UGL & Mercury, total recoverable \\
\hline RWM 7 & & 0.226 & UGL & Mercury, total recoverable \\
\hline RWM 12 & & 0.3105 & UGL & Mercury, total recoverable \\
\hline SBG 1 . & & 0.3365 & UGL & Mercury, total recoverable \\
\hline SRW 2 & $\mathrm{~J}$ & 0.2175 & UGL & Mercury, total recoverable \\
\hline SRW 4 & & 0.26 & UGL & Mercury, total recoverable \\
\hline SRW 6 & $\mathbf{J}$ & 0.165 & UGL & Mercury, total recoverable \\
\hline SRW 17C & $\mathbf{J}$ & 0.169 & UGL & Mercury, total recoverable \\
\hline SSS 6 & & 0.363 & UGL & Mercury, total recoverable \\
\hline SSS 8 & & 0.282 & UGL & Mercury, total recoverable \\
\hline SSS 11 & & 0.37 & UGL & Mercury, total recoverable \\
\hline SSS 20 & & 0.33 & UGL & Mercury, total recoverable \\
\hline SSS 22 & & 0.2165 & UGL & Mercury, total recoverable \\
\hline STR118I & & 0.264 & UGL & Mercury, total recoverable \\
\hline TBG 6 & & 0.392 & UGL & Mercury, total recoverable \\
\hline TBG 7 & $\mathrm{~J}$ & 0.175 & UGL & Mercury, total recoverable \\
\hline TNX 1D & $\mathrm{J}$ & 0.204 & UGL & Mercury, total recoverable \\
\hline TNX 4D & $\mathbf{J}$ & 0.2105 & UGL & Mercury, total recoverable \\
\hline TNX 5D & & 0.2 & UGL & Mercury, total recoverable \\
\hline TNX 6D & $\mathrm{J}$ & 0.248 & UGL & Mercury, total recoverable \\
\hline TNX 13D & $\mathrm{J}$ & 0.16 & UGL & Mercury, total recoverable \\
\hline TRW 2 & $\mathrm{~J}$ & 0.333 & UGL & Mercury, total recoverable \\
\hline
\end{tabular}




\begin{tabular}{|c|c|c|c|c|}
\hline \multicolumn{5}{|c|}{ Wells $(n=272)$ with median mercury concentration estimated between $0.16 \& 0.4 \mathrm{ug} / \mathrm{L}$} \\
\hline well & qualifier & result & units & analyte_name \\
\hline XSB 1A & $\mathrm{J}$ & 0.246 & UGL & Mercury, total recoverable \\
\hline$\overline{\mathrm{XSB}} 1 \mathrm{~B}$ & $\mathrm{~J}$ & 0.202 & UGL & Mercury, total recoverable \\
\hline XSB 2D & $\overline{\mathrm{J}}$ & 0.258 & $\overline{\text { UGL }}$ & Mercury, total recoverable \\
\hline XSB 4D & & 0.289 & UGL & Mercury, total recoverable \\
\hline XSB 5A & $\mathrm{J}$ & 0.256 & $\overline{\text { UGL }}$ & Mercury, total recoverable \\
\hline YSB 3A & $\bar{J}$ & 0.1795 & $\overline{\text { UGL }}$ & Mercury, total recoverable \\
\hline$\overline{Z W ~} 2$ & & 0.3375 & UGL & Mercury, total recoverable \\
\hline
\end{tabular}


Appendix C: Estimated Median Concentrations for SRS Groundwater Wells Associated with the "Impacted" Population.

(total mercury $>0.4 \mathrm{ug} / \mathrm{L}$ ) 


\begin{tabular}{|c|c|c|c|c|}
\hline \multicolumn{5}{|c|}{ Wells $(n=157)$ with median mercury concentration estimated $>0.4 \mathrm{ug} / \mathrm{L}$} \\
\hline well & qualifier & result & units & analyte_name \\
\hline AMB 10B & $\mathrm{J}$ & 0.623 & UGL & Mercury, total recoverable \\
\hline ASB 2C & & 1.12 & UGL & Mercury, total recoverable \\
\hline ASB 3C & & 1.45 & UGL & Mercury, total recoverable \\
\hline $\mathrm{ASB} 3 \mathrm{CR}$ & & 0.644 & UGL & Mercury, total recoverable \\
\hline ASB 8C & & 1.655 & UGL & Mercury, total recoverable \\
\hline ASB 10C & & 1.345 & UGL & Mercury, total recoverable \\
\hline ASB 10CR & & 1.31 & UGL & Mercury, total recoverable \\
\hline BG 55 & & 0.7205 & $\mathrm{UGL}$ & Mercury, total recoverable \\
\hline BG 59 & & 0.88 & UGL & Mercury, total recoverable \\
\hline BG 67 & & 0.5845 & UGL & Mercury, total recoverable \\
\hline $\mathrm{BGO} 33 \mathrm{C}$ & & 3.4 & UGL & Mercury, total recoverable \\
\hline BGO 37C & & 1.89 & UGL & Mercury, total recoverable \\
\hline BGO 46D & & 0.44 & UGL & Mercury, total recoverable \\
\hline BGO 48D & & 0.6755 & $\overline{\mathrm{UGL}}$ & Mercury, total recoverable \\
\hline BRR 7B & & 0.6225 & UGL & Mercury, total recoverable \\
\hline BRR 7B & & 1.14 & UGL & Mercury, total recoverable \\
\hline BRR 7BR & & 2.05 & UGL & Mercury, total recoverable \\
\hline CSD 1D & & 0.608 & UGL & Mercury, total recoverable \\
\hline DBP 2 & & 0.565 & UGL & Mercury, total recoverable \\
\hline DCB 4A & & 0.518 & UGL & Mercury, total recoverable \\
\hline DCB $5 A$ & & 0.442 & UGL & Mercury, total recoverable \\
\hline DCB 6 & & 0.518 & UGL & Mercury, total recoverable \\
\hline DCB 9 & & 0.981 & UGL & Mercury, total recoverable \\
\hline DCB 19B & & 1.09 & UGL & Mercury, total recoverable \\
\hline DCB 19C & & 0.4905 & UGL & Mercury, total recoverable \\
\hline DCB 21B & & 1.485 & UGL & Mercury, total recoverable \\
\hline DCB 21C & & 2.35 & UGL & Mercury, total recoverable \\
\hline DCB 22C & $\mathbf{J}$ & 0.567 & UGL & Mercury, total recoverable \\
\hline DCB 23B & & 4.925 & UGL & Mercury, total recoverable \\
\hline DCB 24C & & 1.34 & UGL & Mercury, total recoverable \\
\hline ERA497A & & 7.37 & UGL & Mercury, total recoverable \\
\hline $\mathrm{F} 14$ & $\mathbf{J}$ & 0.86 & UGL & Mercury, total recoverable \\
\hline F 16 & & 0.51 & UGL & Mercury, total recoverable \\
\hline FCA 9D & & 2.92 & UGL & Mercury, total recoverable \\
\hline FCA 9DR & & 1.34 & UGL & Mercury, total recoverable \\
\hline FNB 2 & & 0.853 & UGL & Mercury, total recoverable \\
\hline FNB 8 & $\mathbf{J}$ & 0.56 & UGL & Mercury, total recoverable \\
\hline FRB 3 & $\mathrm{~J}$ & 0.4065 & UGL & Mercury, total recoverable \\
\hline FSB 77 & & 4.91 & UGL & Mercury, total recoverable \\
\hline FSB 78 & & 0.454 & UGL & Mercury, total recoverable \\
\hline FSB 79 & & 0.7155 & UGL & Mercury, total recoverable \\
\hline FSB 79C & & 0.678 & UGL & Mercury, total recoverable \\
\hline FSB 88D & & 16 & UGL & Mercury, total recoverable \\
\hline FSB 89D & & 6.255 & UGL & Mercury, total recoverable \\
\hline FSB 90D & & 10.045 & $\mathrm{UGL}$ & Mercury, total recoverable \\
\hline FSB 92D & $\mathbf{J}$ & 0.6835 & UGL & Mercury, total recoverable \\
\hline FSB 94DR & & 0.74 & UGL & Mercury, total recoverable \\
\hline FSB 95CR & & 1.18 & UGL & Mercury, total recoverable \\
\hline FSB 95DR & & 0.7785 & UGL & Mercury, total recoverable \\
\hline FSB102C & & 0.546 & UGL & Mercury, total recoverable \\
\hline FSB106C & & 0.7575 & UGL & Mercury, total recoverable \\
\hline FSB 107D & & 4.115 & UGL & Mercury, total recoverable \\
\hline FSB110D & & 0.52 & UGL & Mercury, total recoverable \\
\hline
\end{tabular}




\begin{tabular}{|c|c|c|c|c|}
\hline \multicolumn{5}{|c|}{ Wells $(n=157)$ with median mercury concentration estimated $>0.4 \mathrm{ug} / \mathrm{L}$} \\
\hline well & qualifier & result & units & analyte_name \\
\hline FSB119D & & 0.5605 & UGL & Mercury, total recoverable \\
\hline FSL 9D & & 1.845 & UGL & Mercury, total recoverable \\
\hline FTF 5 & & 0.724 & UGL & Mercury, total recoverable \\
\hline FTF 6 & & 0.688 & UGL & Mercury, total recoverable \\
\hline FTF 7 & & 0.474 & UGL & Mercury, total recoverable \\
\hline FTF 12 & & 1.06 & UGL & Mercury, total recoverable \\
\hline FTF 13 & & 1.59 & UGL & Mercury, total recoverable \\
\hline FTF 20 & & 0.406 & UGL & Mercury, total recoverable \\
\hline FTF 25A & & 0.82 & UGL & Mercury, total recoverable \\
\hline FTF 27 & & 1.055 & UGL & Mercury, total recoverable \\
\hline HAA $1 \mathrm{C}$ & & 0.538 & UGL & Mercury, total recoverable \\
\hline HAC 2 & & 0.664 & UGL & Mercury, total recoverable \\
\hline $\mathrm{HC} \quad 6 \mathrm{~A}$ & & 1.9625 & UGL & Mercury, total recoverable \\
\hline $\mathrm{HCA} 4$ & & 0.757 & UGL & Mercury, total recoverable \\
\hline HET 1D & & 0.939 & UGL & Mercury, total recoverable \\
\hline HEX500TK & & 1.375 & UGL & Mercury, total recoverable \\
\hline HIN600TK & & 0.636 & UGL & Mercury, total recoverable \\
\hline HR3 14DU & & 0.47 & UGL & Mercury, total recoverable \\
\hline HSB 67 & & 1.7 & UGL & Mercury, total recoverable \\
\hline HSB 68 & & 2.25 & UGL & Mercury, total recoverable \\
\hline HSB $68 \mathrm{C}$ & & 0.534 & UGL & Mercury, total recoverable \\
\hline HSB101D & & 4.25 & UGL & Mercury, total recoverable \\
\hline HSB 102C & & 0.477 & UGL & Mercury, total recoverable \\
\hline HSB102D & & 3.735 & UGL & Mercury, total recoverable \\
\hline HSB $103 C$ & & 1.31 & $\overline{\mathrm{UGL}}$ & Mercury, total recoverable \\
\hline HSB 103D & & 1.625 & UGL & Mercury, total recoverable \\
\hline HSB104D & & 2.01 & $\overline{\text { UGL }}$ & Mercury, total recoverable \\
\hline HSB105D & & 4.355 & UGL & Mercury, total recoverable \\
\hline HSB106C & & 0.619 & UGL & Mercury, total recoverable \\
\hline HSB106D & $\mathrm{J}$ & 0.49 & UGL & Mercury, total recoverable \\
\hline HSB107D & & 3.87 & UGL & Mercury, total recoverable \\
\hline HSB108D & & 2.165 & UGL & Mercury, total recoverable \\
\hline HSB109D & & 0.585 & UGL & Mercury, total recoverable \\
\hline HSB116C & & 0.801 & UGL & Mercury, total recoverable \\
\hline HSB125D & & 2.34 & UGL & Mercury, total recoverable \\
\hline HSB126D & & 2.29 & UGL & Mercury, total recoverable \\
\hline HSB127D & & 4.23 & UGL & Mercury, total recoverable \\
\hline HSB134D & & 0.69 & UGL & Mercury, total recoverable \\
\hline HSB139C & & 0.891 & UGL & Mercury, total recoverable \\
\hline HSB145D & & 2.89 & UGL & Mercury, total recoverable \\
\hline HTF 15 & & 1.08 & UGL & Mercury, total recoverable \\
\hline HTF 20 & & 0.609 & UGL & Mercury, total recoverable \\
\hline HTF 21 & & 0.845 & UGL & Mercury, total recoverable \\
\hline HTF 24 & & 0.7895 & UGL & Mercury, total recoverable \\
\hline HTF 26 & & 0.4125 & UGL & Mercury, total recoverable \\
\hline HTF 29 & $\mathrm{~J}$ & 0.648 & UGL & Mercury, total recoverable \\
\hline KBP 1D & & 0.565 & UGL & Mercury, total recoverable \\
\hline LAW 2C & & 0.762 & UGL & Mercury, total recoverable \\
\hline $\operatorname{LCO} 4$ & & 0.5515 & UGL & Mercury, total recoverable \\
\hline LFW 48C & & 2.965 & UGL & Mercury, total recoverable \\
\hline LFW 62C & & 2.16 & UGL & Mercury, total recoverable \\
\hline LFW 62D & & 0.543 & UGL & Mercury, total recoverable \\
\hline LFW 66C & & 0.874 & UGL & Mercury, total recoverable \\
\hline
\end{tabular}




\begin{tabular}{|c|c|c|c|c|}
\hline \multicolumn{5}{|c|}{ Wells $(n=157)$ with median mercury concentration estimated $>0.4 \mathrm{ug} / \mathrm{L}$} \\
\hline well & qualifier & result & units & analyte_name \\
\hline LFW 66D & & 0.548 & UGL & Mercury, total recoverable \\
\hline MGC 19 & & 0.502 & $\overline{U G L}$ & Mercury, total recoverable \\
\hline MGC 23 & & 0.804 & UGL & Mercury, total recoverable \\
\hline MGC 32 & & 3.225 & UGL & Mercury, total recoverable \\
\hline MGE 9 & & 1.68 & UGL & Mercury, total recoverable \\
\hline MGE 21 & & 1.1035 & UGL & Mercury, total recoverable \\
\hline MGE 30 & & 0.41 & UGL & Mercury, total recoverable \\
\hline MGE 34 & & 0.4005 & UGL & Mercury, total recoverable \\
\hline MGG 19 & & 0.484 & UGL & Mercury, total recoverable \\
\hline MGG 23 & & 1.73 & UGL & Mercury, total recoverable \\
\hline MGG 36 & & 3.23 & UGL & Mercury, total recoverable \\
\hline MSB 17A & & 0.691 & UGL & Mercury, total recoverable \\
\hline MSB 26A & & 0.5155 & UGL & Mercury, total recoverable \\
\hline MSB 39B & & 0.4145 & UGL & Mercury, total recoverable \\
\hline MSB 44C & & 1.0215 & UGL & Mercury, total recoverable \\
\hline MSB 70C & & 0.418 & UGL & Mercury, total recoverable \\
\hline MSB 81B & $\mathbf{J}$ & 4.09 & UGL & Mercury, total recoverable \\
\hline NBG 1 & & 0.7935 & UGL & Mercury, total recoverable \\
\hline NBG 2 & & 0.4805 & UGL & Mercury, total recoverable \\
\hline PB 10 & & 3.07 & UGL & Mercury, total recoverable \\
\hline RCP 1D & & 0.641 & $\mathrm{UGL}$ & Mercury, total recoverable \\
\hline RSC 2 & $\mathbf{J}$ & 0.929 & UGL & Mercury, total recoverable \\
\hline RSC 5 & & 0.724 & UGL & Mercury, total recoverable \\
\hline RSD 6 & & 0.4105 & UGL & Mercury, total recoverable \\
\hline RSE 4A & & 0.5065 & UGL & Mercury, total recoverable \\
\hline RSE 5 & & 1.37 & UGL & Mercury, total recoverable \\
\hline RSE 6 & & 2.45 & UGL & Mercury, total recoverable \\
\hline RSE 18 & & 1.1275 & UGL & Mercury, total recoverable \\
\hline RSE 19 & & 0.4125 & UGL & Mercury, total recoverable \\
\hline RSP 1D & $\mathrm{J}$ & 0.7 & UGL & Mercury, total recoverable \\
\hline RWM 1 & & 0.421 & UGL & Mercury, total recoverable \\
\hline SSS 1 & & 0.477 & UGL & Mercury, total recoverable \\
\hline SSS 5 & & 0.654 & UGL & Mercury, total recoverable \\
\hline SSS 7 & & 0.522 & UGL & Mercury, total recoverable \\
\hline SSS 10 & & 2.96 & UGL & Mercury, total recoverable \\
\hline SSS 12 & & 1.08 & UGL & Mercury, total recoverable \\
\hline SSS 27 & & 0.681 & UGL & Mercury, total recoverable \\
\hline TBG 3 & $\mathbf{J}$ & 0.543 & UGL & Mercury, total recoverable \\
\hline TBG 4 & & 2.165 & UGL & Mercury, total recoverable \\
\hline TIR 3B & $\mathbf{J}$ & 0.4295 & UGL & Mercury, total recoverable \\
\hline TNX 3D & & 0.97 & UGL & Mercury, total recoverable \\
\hline TRW 1 & $\mathbf{J}$ & 0.528 & UGL & Mercury, total recoverable \\
\hline $\mathrm{XSB} 3 \mathrm{~A}$ & & 0.526 & UGL & Mercury, total recoverable \\
\hline $\mathrm{Z} 9$ & & 0.616 & UGL & Mercury, total recoverable \\
\hline ZW 3 & & 0.72 & UGL & Mercury, total recoverable \\
\hline ZW 4 & & 0.966 & UGL & Mercury, total recoverable \\
\hline ZW 5 & $\mathrm{~J}$ & 2.34 & UGL & Mercury, total recoverable \\
\hline ZW 6 & & 1.625 & UGL & Mercury, total recoverable \\
\hline ZW 7 & & 0.479 & UGL & Mercury, total recoverable \\
\hline ZW 9 & & 0.665 & UGL & Mercury, total recoverable \\
\hline ZW 10 & & 1.225 & UGL & Mercury, total recoverable \\
\hline
\end{tabular}


Appendix D: SRS Groundwater Wells with all Reported Values Below Nominal Detection Limit. 
Wells $(n=856)$ with no estimated median mercury concentration (all values nondetect at or below nominal D.L.)

\begin{tabular}{|c|c|c|c|c|}
\hline well & qualifier & result & units & analyte_name \\
\hline $\mathrm{ABP} 2 \mathrm{~A}$ & $\mathrm{U}$ & 0.2 & UGL & Mercury, total recoverable \\
\hline ABP 3C & $\mathrm{U}$ & 0.2 & UGL & Mercury, total recoverable \\
\hline $\mathrm{ABP} 4$ & $\mathrm{U}$ & 0.2 & UGL & Mercury, total recoverable \\
\hline $\mathrm{ABP} 8 \mathrm{C}$ & U & 0.2 & UGL & Mercury, total recoverable \\
\hline $\mathrm{ABW} 1$ & $\mathrm{U}$ & 0.2 & UGL & Mercury, total recoverable \\
\hline $\mathrm{AC} \quad 1 \mathrm{~A}$ & $\mathrm{U}$ & 0.2 & UGL & Mercury, total recoverable \\
\hline $\mathrm{AC} \quad 1 \mathrm{~B}$ & $\mathrm{U}$ & 0.2 & UGL & Mercury, total recoverable \\
\hline $\mathrm{AC} 2 \mathrm{~A}$ & $\mathrm{U}$ & 0.2 & UGL & Mercury, total recoverable \\
\hline $\mathrm{AC} \quad 2 \mathrm{~B}$ & $\mathrm{U}$ & 0.2 & UGL & Mercury, total recoverable \\
\hline $\mathrm{AC} \quad 3 \mathrm{~B}$ & $\mathrm{U}$ & 0.2 & UGL & Mercury, total recoverable \\
\hline $\mathrm{ACB} 1 \mathrm{~A}$ & $\mathrm{U}$ & 0.2 & UGL & Mercury, total recoverable \\
\hline $\mathrm{AMB} 4 \mathrm{~B}$ & $\mathrm{U}$ & 0.2 & UGL & Mercury, total recoverable \\
\hline AMB 4D & $\mathrm{U}$ & 0.2 & UGL & Mercury, total recoverable \\
\hline AMB 10D & $\bar{U}$ & 0.2 & UGL & Mercury, total recoverable \\
\hline AMB 12D & $\mathrm{U}$ & 0.2 & UGL & Mercury, total recoverable \\
\hline $\mathrm{AOB} 1$ & $\mathrm{U}$ & 0.2 & UGL & Mercury, total recoverable \\
\hline $\mathrm{AOB} 2$ & $\mathrm{U}$ & 0.2 & UGL & Mercury, total recoverable \\
\hline $\mathrm{AOB} 2$ & $\mathrm{U}$ & 0.2 & UGL & Mercury, total recoverable \\
\hline $\mathrm{AOB} 3$ & $\mathrm{U}$ & 0.2 & UGL & Mercury, total recoverable \\
\hline ARP 2 & $\mathrm{U}$ & 0.2 & UGL & Mercury, total recoverable \\
\hline ARP 3 & $\mathrm{U}$ & 0.2 & UGL & Mercury, total recoverable \\
\hline ARP 4 & $\mathrm{U}$ & 0.2 & UGL & Mercury, total recoverable \\
\hline ASB 2A & $\mathrm{U}$ & 0.2 & UGL & Mercury, total recoverable \\
\hline ASB 3A & $\mathrm{U}$ & 0.2 & UGL & Mercury, total recoverable \\
\hline ASB 5A & $\mathbf{U}$ & 0.2 & UGL & Mercury, total recoverable \\
\hline ASB 5AR & $\mathbf{U}$ & 0.2 & UGL & Mercury, total recoverable \\
\hline ASB 6TA & $\mathbf{U}$ & 0.2 & UGL & Mercury, total recoverable \\
\hline ASB 7 & $\mathbf{U}$ & 0.2 & UGL & Mercury, total recoverable \\
\hline ASB 9C & $\mathrm{U}$ & 0.2 & UGL & Mercury, total recoverable \\
\hline BG 60 & $\mathrm{U}$ & 0.2 & UGL & Mercury, total recoverable \\
\hline BG 61 & $\mathrm{U}$ & 0.2 & UGL & Mercury, total recoverable \\
\hline BG 91 & $\mathrm{U}$ & 0.2 & UGL & Mercury, total recoverable \\
\hline BG 92 & $\mathbf{U}$ & 0.2 & UGL & Mercury, total recoverable \\
\hline BG 93 & $\mathbf{U}$ & 0.2 & UGL & Mercury, total recoverable \\
\hline BG 94 & $\mathbf{U}$ & 0.2 & UGL & Mercury, total recoverable \\
\hline BG 95 & $\mathrm{U}$ & 0.2 & UGL & Mercury, total recoverable \\
\hline BG 96 & U & 0.2 & UGL & Mercury, total recoverable \\
\hline BG 101 & $\mathrm{U}$ & 0.2 & UGL & Mercury, total recoverable \\
\hline BG 103 & $\mathrm{U}$ & 0.2 & UGL & Mercury, total recoverable \\
\hline BG 104 & $\mathrm{U}$ & 0.2 & UGL & Mercury, total recoverable \\
\hline BG 107 & $\mathrm{U}$ & 0.2 & UGL & Mercury, total recoverable \\
\hline BG 108 & $\mathrm{U}$ & 0.2 & UGL & Mercury, total recoverable \\
\hline BG 109 & $\mathrm{U}$ & 0.2 & UGL & Mercury, total recoverable \\
\hline $\mathrm{BG} 110$ & $\mathrm{U}$ & 0.2 & UGL & Mercury, total recoverable \\
\hline BG 122 & $\mathrm{U}$ & 0.2 & UGL & Mercury, total recoverable \\
\hline $\mathrm{BGO} 3 \mathrm{~A}$ & $\mathrm{U}$ & 0.2 & UGL & Mercury, total recoverable \\
\hline $\mathrm{BGO} 3 \mathrm{C}$ & $\underline{U}$ & 0.2 & UGL & Mercury, total recoverable \\
\hline BGO 3D & $\mathrm{U}$ & 0.2 & UGL & Mercury, total recoverable \\
\hline BGO 5C & $\mathrm{U}$ & 0.2 & UGL & Mercury, total recoverable \\
\hline BGO 5D & $\mathbf{U}$ & 0.2 & UGL & Mercury, total recoverable \\
\hline $\mathrm{BGO} 6 \mathrm{~B}$ & $\mathrm{U}$ & 0.2 & UGL & Mercury, total recoverable \\
\hline BGO $6 \mathrm{C}$ & $\mathrm{U}$ & 0.2 & UGL & Mercury, total recoverable \\
\hline
\end{tabular}




\begin{tabular}{|c|c|c|c|c|}
\hline Wells $(n=856$ & with no es & $\begin{array}{l}\text { d medi } \\
\text { belo }\end{array}$ & $\begin{array}{l}\text { rcury c } \\
\text { minal D }\end{array}$ & ntration (all values nondet \\
\hline well & qualifier & result & units & analyte_name \\
\hline $\mathrm{BGO} 6 \mathrm{D}$ & $\mathrm{U}$ & 0.2 & UGL & Mercury, total recoverable \\
\hline BGO 9AA & $\mathrm{U}$ & 0.2 & UGL & Mercury, total recoverable \\
\hline BGO 10B & $\mathrm{U}$ & 0.2 & UGL & Mercury, total recoverable \\
\hline $\mathrm{BGO} 10 \mathrm{C}$ & $\mathrm{U}$ & 0.2 & UGL & Mercury, total recoverable \\
\hline BGO 11D & $\mathrm{U}$ & 0.2 & UGL & Mercury, total recoverable \\
\hline BGO 12AR & $\mathrm{U}$ & 0.2 & UGL & Mercury, total recoverable \\
\hline BGO 12CR & $\bar{U}$ & 0.2 & UGL & Mercury, total recoverable \\
\hline BGO 12D & $\mathrm{U}$ & 0.2 & UGL & Mercury, total recoverable \\
\hline BGO 12DR & $\mathrm{U}$ & 0.2 & UGL & Mercury, total recoverable \\
\hline BGO 13DR & $\overline{\mathrm{U}}$ & 0.2 & UGL & Mercury, total recoverable \\
\hline BGO 15D & $\bar{U}$ & 0.2 & UGL & Mercury, total recoverable \\
\hline BGO 16A & $U$ & 0.2 & UGL & Mercury, total recoverable \\
\hline BGO 16D & $\mathrm{U}$ & 0.2 & UGL & Mercury, total recoverable \\
\hline BGO 17DR & $\overline{\mathrm{U}}$ & 0.2 & $\overline{\text { UGL }}$ & Mercury, total recoverable \\
\hline BGO 18A & $\bar{U}$ & 0.2 & $\overline{\text { UGL }}$ & Mercury, total recoverable \\
\hline BGO 18D & $\mathrm{U}$ & 0.2 & UGL & Mercury, total recoverable \\
\hline BGO 19D & $\bar{U}$ & 0.2 & UGL & Mercury, total recoverable \\
\hline$\overline{B G O} 19 D R$ & $\overline{\mathrm{U}}$ & 0.2 & UGL & Mercury, total recoverable \\
\hline BGO 22D & $\mathrm{U}$ & 0.2 & UGL & Mercury, total recoverable \\
\hline BGO 22DR & $\mathrm{U}$ & 0.2 & UGL & Mercury, total recoverable \\
\hline BGO 22DX & $\mathrm{U}$ & 0.2 & UGL & Mercury, total recoverable \\
\hline BGO 24D & $\mathrm{U}$ & 0.2 & UGL & Mercury, total recoverable \\
\hline BGO 25A & $\mathrm{U}$ & 0.2 & UGL & Mercury, total recoverable \\
\hline BGO 26A & $\mathrm{U}$ & 0.2 & UGL & Mercury, total recoverable \\
\hline BGO 26D & $\mathbf{U}$ & 0.2 & UGL & Mercury, total recoverable \\
\hline BGO 27C & $\bar{U}$ & 0.2 & UGL & Mercury, total recoverable \\
\hline $\mathrm{BGO} 29 \mathrm{~A}$ & $\mathrm{U}$ & 0.2 & UGL & Mercury, total recoverable \\
\hline BGO 29C & $\mathrm{U}$ & 0.2 & $\overline{\text { UGL }}$ & Mercury, total recoverable \\
\hline BGO 29D & $\mathrm{U}$ & 0.2 & UGL & Mercury, total recoverable \\
\hline BGO 31C & $\mathrm{U}$ & 0.2 & UGL & Mercury, total recoverable \\
\hline BGO 34D & $\bar{U}$ & 0.2 & UGL & Mercury, total recoverable \\
\hline BGO 36D & $\mathrm{U}$ & 0.2 & UGL & Mercury, total recoverable \\
\hline BGO 38D & $\overline{\mathrm{U}}$ & 0.2 & UGL & Mercury, total recoverable \\
\hline BGO 39C & $\mathrm{U}$ & 0.2 & UGL & Mercury, total recoverable \\
\hline BGO 39D & $\mathrm{U}$ & 0.2 & UGL & Mercury, total recoverable \\
\hline $\mathrm{BGO} 40 \mathrm{D}$ & $\mathrm{U}$ & 0.2 & UGL & Mercury, total recoverable \\
\hline $\mathrm{BGO} 41 \mathrm{~A}$ & $\bar{U}$ & 0.2 & UGL & Mercury, total recoverable \\
\hline BGO 42C & $\mathrm{U}$ & 0.2 & UGL & Mercury, total recoverable \\
\hline $\mathrm{BGO} 43 \mathrm{~A}$ & $\mathrm{U}$ & 0.2 & UGL & Mercury, total recoverable \\
\hline $\mathrm{BGO} 43 \mathrm{AA}$ & $\mathrm{U}$ & 0.2 & UGL & Mercury, total recoverable \\
\hline BGO 43CR & $\mathrm{U}$ & 0.2 & UGL & Mercury, total recoverable \\
\hline BGO 43D & $\bar{U}$ & 0.2 & UGL & Mercury, total recoverable \\
\hline $\mathrm{BGO} 44 \mathrm{~B}$ & $\mathrm{U}$ & 0.2 & UGL & Mercury, total recoverable \\
\hline BGO 44C & $\mathrm{U}$ & 0.2 & UGL & Mercury, total recoverable \\
\hline BGO 44D & $\mathrm{U}$ & 0.2 & UGL & Mercury, total recoverable \\
\hline BGO 45A & $\mathrm{U}$ & 0.2 & UGL & Mercury, total recoverable \\
\hline $\mathrm{BGO} 45 \mathrm{~B}$ & $\mathrm{U}$ & 0.2 & UGL & Mercury, total recoverable \\
\hline BGO 45C & $\mathrm{U}$ & 0.2 & UGL & Mercury, total recoverable \\
\hline BGO 45D & $\mathrm{U}$ & 0.2 & UGL & Mercury, total recoverable \\
\hline BGO 46B & $\mathrm{U}$ & 0.2 & UGL & Mercury, total recoverable \\
\hline $\mathrm{BGO} 47 \mathrm{C}$ & $\mathrm{U}$ & 0.2 & UGL & Mercury, total recoverable \\
\hline BGO 47D & $\bar{U}$ & 0.2 & UGL & Mercury, total recoverable \\
\hline
\end{tabular}




\begin{tabular}{|c|c|c|c|c|}
\hline Wells $(\mathrm{n}=85$ & with no es & $\begin{array}{r}\text { ed media } \\
\text { belo } \\
\end{array}$ & $\begin{array}{l}\text { rcury } \\
\text { minal D } \\
\end{array}$ & ntration (all values nondetect at on \\
\hline well & qualifier & result & units & analyte_name \\
\hline $\mathrm{BGO} 48 \mathrm{C}$ & $\mathrm{U}$ & 0.2 & UGL & Mercury, total recoverable \\
\hline BGO 49A & $\mathrm{U}$ & 0.2 & UGL & Mercury, total recoverable \\
\hline $\mathrm{BGO} 50 \mathrm{C}$ & $\mathrm{U}$ & 0.2 & UGL & Mercury, total recoverable \\
\hline BGO 50D & $\mathrm{U}$ & 0.2 & UGL & Mercury, total recoverable \\
\hline $\mathrm{BGO} 53 \mathrm{AA}$ & $\mathrm{U}$ & 0.2 & UGL & Mercury, total recoverable \\
\hline BGO 53C & $\mathrm{U}$ & 0.2 & UGL & Mercury, total recoverable \\
\hline BGX 1A & $\mathrm{U}$ & 0.2 & UGL & Mercury, total recoverable \\
\hline BGX 1C & $\mathrm{U}$ & 0.2 & UGL & Mercury, total recoverable \\
\hline $\mathrm{BGX} 1 \mathrm{D}$ & $\mathrm{U}$ & 0.2 & UGL & Mercury, total recoverable \\
\hline BGX 2D & $\mathrm{U}$ & 0.2 & UGL & Mercury, total recoverable \\
\hline $\mathrm{BGX} 6 \mathrm{D}$ & $\mathrm{U}$ & 0.2 & UGL & Mercury, total recoverable \\
\hline $\mathrm{BGX} 7 \mathrm{D}$ & $\mathrm{U}$ & 0.2 & UGL & Mercury, total recoverable \\
\hline BGX 8DR & $\mathbf{U}$ & 0.2 & UGL & Mercury, total recoverable \\
\hline BGX 9D & $\mathrm{U}$ & 0.2 & UGL & Mercury, total recoverable \\
\hline $\mathrm{BGX} 10 \mathrm{D}$ & $\mathrm{U}$ & 0.2 & UGL & Mercury, total recoverable \\
\hline BGX 11D & $\mathrm{U}$ & 0.2 & UGL & Mercury, total recoverable \\
\hline BRD 1 & $\mathrm{U}$ & 0.2 & UGL & Mercury, total recoverable \\
\hline BRD 2 & $\mathrm{U}$ & 0.2 & UGL & Mercury, total recoverable \\
\hline BRD 3 & $\mathbf{U}$ & 0.2 & UGL & Mercury, total recoverable \\
\hline BRD 4 & $\mathrm{U}$ & 0.2 & UGL & Mercury, total recoverable \\
\hline BRD 5D & $\mathrm{U}$ & 0.2 & UGL & Mercury, total recoverable \\
\hline BRR 7C & $\mathrm{U}$ & 0.2 & UGL. & Mercury, total recoverable \\
\hline BRR 8DR & U & 0.2 & UGL & Mercury, total recoverable \\
\hline CBR 2D & $\mathrm{U}$ & 0.2 & UGL & Mercury, total recoverable \\
\hline CBR 3D & $\mathrm{U}$ & 0.2 & UGL & Mercury, total recoverable \\
\hline CCB 4 & $\mathrm{U}$ & 0.2 & UGL & Mercury, total recoverable \\
\hline CDB 1 & $\mathrm{U}$ & 0.2 & UGL & Mercury, total recoverable \\
\hline CDB 2 & $\mathrm{U}$ & 0.2 & UGL & Mercury, total recoverable \\
\hline CMP 9B & U & 0.2 & UGL & Mercury, total recoverable \\
\hline CMP 10 & $\mathrm{U}$ & 0.2 & UGL & Mercury, total recoverable \\
\hline CMP 11 & $\mathrm{U}$ & 0.2 & UGL & Mercury, total recoverable \\
\hline CMP 11B & $\mathrm{U}$ & 0.2 & UGL & Mercury, total recoverable \\
\hline CMP 14B & $\mathbf{U}$ & 0.2 & UGL & Mercury, total recoverable \\
\hline CMP 14D & $\mathrm{U}$ & 0.2 & UGL & Mercury, total recoverable \\
\hline CMP 30B & $\mathrm{U}$ & 0.059 & UGL & Mercury, total recoverable \\
\hline CMP 31C & $\mathrm{U}$ & 0.2 & UGL & Mercury, total recoverable \\
\hline CMP 32B & $\mathrm{U}$ & 0.2 & UGL & Mercury, total recoverable \\
\hline CRP 1 & $\mathrm{U}$ & 0.2 & UGL & Mercury, total recoverable \\
\hline CRP 2 & $\mathrm{U}$ & 0.2 & UGL & Mercury, total recoverable \\
\hline CRP 11D & $\mathrm{U}$ & 0.2 & UGL & Mercury, total recoverable \\
\hline CSA 1 & $\mathrm{U}$ & 0.2 & UGL & Mercury, total recoverable \\
\hline CSA 2 & $\mathrm{U}$ & 0.2 & UGL & Mercury, total recoverable \\
\hline CSA 3 & $\mathrm{U}$ & 0.2 & UGL & Mercury, total recoverable \\
\hline CSA 4 & $\mathrm{U}$ & 0.2 & UGL & Mercury, total recoverable \\
\hline CSB 1A & $\mathrm{U}$ & 0.7 & UGL & Mercury, total recoverable \\
\hline CSB 1C & $\mathrm{U}$ & 0.2 & UGL & Mercury, total recoverable \\
\hline CSB 2A & $\mathrm{U}$ & 0.2 & UGL & Mercury, total recoverable \\
\hline CSB 3A & $\mathrm{U}$ & 0.2 & UGL & Mercury, total recoverable \\
\hline $\mathrm{CSB} 3 \mathrm{C}$ & U & 0.2 & UGL & Mercury, total recoverable \\
\hline CSB 4A & $\mathrm{U}$ & 0.2 & UGL & Mercury, total recoverable \\
\hline CSB 5A & $\mathrm{U}$ & 0.2 & UGL & Mercury, total recoverable \\
\hline CSB 7D & $\mathrm{U}$ & 0.2 & UGL & Mercury, total recoverable \\
\hline
\end{tabular}




\begin{tabular}{|c|c|c|c|c|}
\hline \multicolumn{5}{|c|}{$\begin{array}{r}\text { Wells ( } \mathrm{n}=856 \text { ) with no estimated median mercury conce } \\
\text { below nominal D.L.) }\end{array}$} \\
\hline well & qualifier & result & units & analyte_name \\
\hline $\mathrm{CSD} 2 \mathrm{D}$ & $\mathrm{U}$ & 0.2 & UGL & Mercury, total recoverable \\
\hline $\mathrm{CSD} 4 \mathrm{D}$ & $\mathrm{U}$ & 0.2 & UGL & Mercury, total recoverable \\
\hline CSD 8D & $\mathrm{U}$ & 0.2 & UGL & Mercury, total recoverable \\
\hline CSD 9D & $\mathrm{U}$ & 0.2 & UGL & Mercury, total recoverable \\
\hline CSD 10D & $\mathrm{U}$ & 0.2 & UGL & Mercury, total recoverable \\
\hline CSD 11D & $\overline{\mathrm{U}}$ & 0.2 & UGL & Mercury, total recoverable \\
\hline CSD 12D & $\mathrm{U}$ & 0.2 & UGL & Mercury, total recoverable \\
\hline $\mathrm{CSD} 12 \mathrm{D}$ & $\mathrm{U}$ & 0.2 & UGL & Mercury, total recoverable \\
\hline CSD 13D & $\mathrm{U}$ & 0.2 & UGL & Mercury, total recoverable \\
\hline $\mathrm{CSO} 1$ & $\bar{U}$ & 0.2 & UGL & Mercury, total recoverable \\
\hline $\mathrm{CSO} 2$ & $\mathrm{U}$ & 0.2 & UGL & Mercury, total recoverable \\
\hline CSR 1 & $\mathrm{U}$ & 0.2 & UGL & Mercury, total recoverable \\
\hline CSR 2 & $\mathrm{U}$ & 0.2 & UGL & Mercury, total recoverable \\
\hline CSR 2D & $\bar{U}$ & 0.2 & UGL & Mercury, total recoverable \\
\hline CSR 3 & $\mathbf{U}$ & 0.2 & UGL & Mercury, total recoverable \\
\hline CSR 4 & $\mathrm{U}$ & 0.2 & UGL & Mercury, total recoverable \\
\hline CSR 7DL & $\mathrm{U}$ & 0.2 & UGL & Mercury, total recoverable \\
\hline CSR 7DU & $\mathrm{U}$ & 0.2 & UGL & Mercury, total recoverable \\
\hline CSR 8DL & $\mathrm{U}$ & 0.2 & UGL & Mercury, total recoverable \\
\hline CSR 8DU & $\mathrm{U}$ & 0.2 & UGL & Mercury, total recoverable \\
\hline CSR 9DL & $\mathbf{U}$ & 0.2 & UGL & Mercury, total recoverable \\
\hline CSR 9DU & $\mathrm{U}$ & 0.2 & UGL & Mercury, total recoverable \\
\hline CSR 10DL & $\mathrm{U}$ & 0.2 & UGL & Mercury, total recoverable \\
\hline CSR 10DU & $\mathbf{U}$ & 0.2 & UGL & Mercury, total recoverable \\
\hline CSR 11DL & $\mathrm{U}$ & 0.2 & UGL & Mercury, total recoverable \\
\hline CSR 11DU & $\mathrm{U}$ & 0.2 & UGL & Mercury, total recoverable \\
\hline DBP 1 & $\mathrm{U}$ & 0.2 & UGL & Mercury, total recoverable \\
\hline DBP 3 & $\mathrm{U}$ & 0.2 & UGL & Mercury, total recoverable \\
\hline DBP 4 & $\mathrm{U}$ & 0.2 & UGL & Mercury, total recoverable \\
\hline DBP 5 & $\mathrm{U}$ & 0.2 & UGL & Mercury, total recoverable \\
\hline DCB 2A & $\mathrm{U}$ & 0.2 & UGL & Mercury, total recoverable \\
\hline DCB $3 \mathrm{~A}$ & $\mathrm{U}$ & 0.2 & UGL & Mercury, total recoverable \\
\hline DCB 8 & $\mathrm{U}$ & 0.2 & UGL & Mercury, total recoverable \\
\hline DCB 11 & $\mathrm{U}$ & 0.2 & UGL & Mercury, total recoverable \\
\hline DCB 12 & $\mathrm{U}$ & 0.2 & UGL & Mercury, total recoverable \\
\hline DCB 13 & $\mathrm{U}$ & 0.2 & UGL & Mercury, total recoverable \\
\hline DCB 15 & $\mathrm{U}$ & 0.2 & UGL & Mercury, total recoverable \\
\hline DCB 16 & $\mathrm{U}$ & 0.2 & UGL & Mercury, total recoverable \\
\hline DCB 16R & $\mathrm{U}$ & 0.2 & UGL & Mercury, total recoverable \\
\hline DCB 19A & $\mathrm{U}$ & 0.2 & UGL & Mercury, total recoverable \\
\hline DOB 3 & $\mathbf{U}$ & 0.2 & UGL & Mercury, total recoverable \\
\hline DOB 4 & $\mathbf{U}$ & 0.2 & UGL & Mercury, total recoverable \\
\hline DOB 8 & $\mathrm{U}$ & 0.2 & UGL & Mercury, total recoverable \\
\hline DOB 9 & $\mathbf{U}$ & 0.2 & UGL & Mercury, total recoverable \\
\hline DOB 11 & $\mathbf{U}$ & 0.076 & UGL & Mercury, total recoverable \\
\hline DOB 11 & $\mathrm{U}$ & 0.2 & UGL & Mercury, total recoverable \\
\hline DOB 13 & $\mathrm{U}$ & 0.2 & UGL & Mercury, total recoverable \\
\hline DOB 14 & $\mathrm{U}$ & 0.2 & UGL & Mercury, total recoverable \\
\hline DOB 15 & $\mathrm{U}$ & 0.2 & UGL & Mercury, total recoverable \\
\hline DOL 1 & $\mathrm{U}$ & 0.2 & UGL & Mercury, total recoverable \\
\hline DOL 1 & $\mathrm{U}$ & 0.2 & UGL & Mercury, total recoverable \\
\hline DOL 1 & $\mathrm{U}$ & 0.2 & UGL & Mercury, total recoverable \\
\hline
\end{tabular}


$\overline{\text { Wells }(\mathrm{n}=856 \text { ) with no estimated median mercury concentration (all values nondetect at or }}$ below nominal D.L.)

\begin{tabular}{|c|c|c|c|c|}
\hline well & qualifier & result & units & analyte_name \\
\hline FAB 3 & $\mathrm{U}$ & 0.2 & UGL & Mercury, total recoverable \\
\hline FAC $9 \mathrm{C}$ & $\mathrm{U}$ & 0.2 & UGL & Mercury, total recoverable \\
\hline FAC $10 \mathrm{C}$ & $\mathrm{U}$ & 0.2 & UGL & Mercury, total recoverable \\
\hline FAC 12C & $\mathrm{U}$ & 0.2 & UGL & Mercury, total recoverable \\
\hline FBP 1A & $U$ & 0.2 & UGL & Mercury, total recoverable \\
\hline FBP 3A & $\mathrm{U}$ & 0.2 & UGL & Mercury, total recoverable \\
\hline FBP 4 & $\mathrm{U}$ & 0.2 & UGL & Mercury, total recoverable \\
\hline FBP 5D & $\mathrm{U}$ & 0.2 & UGL & Mercury, total recoverable \\
\hline FBP 6D & $\mathrm{U}$ & 0.2 & UGL & Mercury, total recoverable \\
\hline FBP 8D & $\mathrm{U}$ & 0.2 & UGL & Mercury, total recoverable \\
\hline FBP 9D & $\mathrm{U}$ & 0.2 & UGL & Mercury, total recoverable \\
\hline FBP 12D & $\mathrm{U}$ & 0.2 & UGL & Mercury, total recoverable \\
\hline FBP 13D & $\mathrm{U}$ & 0.2 & UGL & Mercury, total recoverable \\
\hline FCA 1N & $\mathrm{U}$ & 0.2 & UGL & Mercury, total recoverable \\
\hline FCA 2D & U & 0.2 & UGL & Mercury, total recoverable \\
\hline FCA $10 \mathrm{C}$ & $\mathrm{U}$ & 0.2 & UGL & Mercury, total recoverable \\
\hline FCA 10D & $\mathrm{U}$ & 0.2 & UGL & Mercury, total recoverable \\
\hline FCA $16 \mathrm{~B}$ & $\mathrm{U}$ & 0.2 & UGL & Mercury, total recoverable \\
\hline FCA 16D & $\mathrm{U}$ & 0.5185 & UGL & Mercury, total recoverable \\
\hline FCA 19D & $\mathrm{U}$ & 0.2 & UGL & Mercury, total recoverable \\
\hline FCB 2 & $\mathrm{U}$ & 0.2 & UGL & Mercury, total recoverable \\
\hline FCB 3 & $\mathrm{U}$ & 0.2 & UGL & Mercury, total recoverable \\
\hline FCB 5 & $\mathrm{U}$ & 0.2 & UGL & Mercury, total recoverable \\
\hline FCB 6 & $\mathrm{U}$ & 0.2 & UGL & Mercury, total recoverable \\
\hline FET 1D & $\mathrm{U}$ & 0.7 & UGL & Mercury, total recoverable \\
\hline FET 2D & $\mathrm{U}$ & 0.7 & UGL & Mercury, total recoverable \\
\hline FET 3D & $\mathrm{U}$ & 0.2 & UGL & Mercury, total recoverable \\
\hline FET 4D & $\mathrm{U}$ & 0.2 & UGL & Mercury, total recoverable \\
\hline FEX 11 & $\mathrm{U}$ & 0.2 & UGL & Mercury, total recoverable \\
\hline FNB 1A & $\mathrm{U}$ & 0.2 & UGL & Mercury, total recoverable \\
\hline FNB 2A & $\mathbf{U}$ & 0.2 & UGL & Mercury, total recoverable \\
\hline FNB 3A & $\mathbf{U}$ & 0.2 & UGL & Mercury, total recoverable \\
\hline FNB 4 & $\mathrm{U}$ & 0.2 & UGL & Mercury, total recoverable \\
\hline FRB 1 & $\mathbf{U}$ & 0.2 & UGL & Mercury, total recoverable \\
\hline FSB 1TA & $\mathrm{U}$ & 0.2 & UGL & Mercury, total recoverable \\
\hline FSB 76A & $\mathbf{U}$ & 0.2 & UGL & Mercury, total recoverable \\
\hline FSB 76B & $\mathrm{U}$ & 0.2 & UGL & Mercury, total recoverable \\
\hline FSB 76C & $\mathrm{U}$ & 0.2 & UGL & Mercury, total recoverable \\
\hline FSB 78A & $\mathrm{U}$ & 0.2 & UGL & Mercury, total recoverable \\
\hline FSB 78B & $\mathrm{U}$ & 0.2 & UGL & Mercury, total recoverable \\
\hline FSB 79A & $\mathrm{U}$ & 0.2 & UGL & Mercury, total recoverable \\
\hline FSB 87A & $\mathrm{U}$ & 0.2 & UGL & Mercury, total recoverable \\
\hline FSB 87B & $\mathrm{U}$ & 0.2 & UGL & Mercury, total recoverable \\
\hline FSB 88C & $\mathrm{U}$ & 0.2 & UGL & Mercury, total recoverable \\
\hline FSB 89C & $\mathrm{U}$ & 0.2 & UGL & Mercury, total recoverable \\
\hline FSB 90C & $\mathrm{U}$ & 0.2 & UGL & Mercury, total recoverable \\
\hline FSB 91C & $\mathrm{U}$ & 0.2 & UGL & Mercury, total recoverable \\
\hline FSB 92C & $\mathrm{U}$ & 0.2 & UGL & Mercury, total recoverable \\
\hline FSB 94C & $\mathrm{U}$ & 0.2 & UGL & Mercury, total recoverable \\
\hline FSB 99A & $\mathrm{U}$ & 0.2 & UGL & Mercury, total recoverable \\
\hline FSB 99D & $\mathrm{U}$ & 0.2 & UGL & Mercury, total recoverable \\
\hline FSB100A & $\mathrm{U}$ & 0.2 & UGL & Mercury, total recoverable \\
\hline
\end{tabular}




\begin{tabular}{|c|c|c|c|c|}
\hline \multicolumn{5}{|c|}{$\begin{array}{l}\text { Wells ( } \mathrm{n}=856 \text { ) with no estimated median mercury concentration (all values nondetect at or } \\
\text { below nominal D.L.) }\end{array}$} \\
\hline well & qualifier & result & units & \begin{tabular}{|c|} 
analyte_name \\
\end{tabular} \\
\hline FSB101A & $\mathrm{U}$ & 0.2 & UGL & Mercury, total recoverable \\
\hline FSB 103C & $\mathrm{U}$ & 0.2 & UGL & Mercury, total recoverable \\
\hline FSB 106D & $\mathrm{U}$ & 0.2 & UGL & Mercury, total recoverable \\
\hline FSB 107C & $\mathrm{U}$ & 0.2 & UGL & Mercury, total recoverable \\
\hline FSB113A & $\mathrm{U}$ & 0.2 & UGL & Mercury, total recoverable \\
\hline FSB 113C & $\mathrm{U}$ & 0.2 & UGL & Mercury, total recoverable \\
\hline FSB114A & $\mathrm{U}$ & 0.2 & UGL & Mercury, total recoverable \\
\hline FSB114D & $\mathrm{U}$ & 0.2 & UGL & Mercury, total recoverable \\
\hline FSB $115 \mathrm{C}$ & $\mathrm{U}$ & 0.2 & UGL & Mercury, total recoverable \\
\hline FSB115D & $\mathrm{U}$ & 0.2 & UGL & Mercury, total recoverable \\
\hline FSB 116D & $\mathrm{U}$ & 0.2 & UGL & Mercury, total recoverable \\
\hline FSB 117D & $\mathrm{U}$ & 0.2 & UGL & Mercury, total recoverable \\
\hline FSB120A & $\mathrm{U}$ & 0.2 & UGL & Mercury, total recoverable \\
\hline FSB 120D & $\mathrm{U}$ & 0.2 & UGL & Mercury, total recoverable \\
\hline FSB122C & $\mathrm{U}$ & 0.2 & UGL & Mercury, total recoverable \\
\hline FSB123C & $\mathrm{U}$ & 0.2 & UGL & Mercury, total recoverable \\
\hline FSB 123D & $U$ & 0.2 & UGL & Mercury, total recoverable \\
\hline FSL 3D & $\mathrm{U}$ & 0.2 & UGL & Mercury, total recoverable \\
\hline FSL 4D & $\mathrm{U}$ & 0.2 & UGL & Mercury, total recoverable \\
\hline FTF 2 & $\tilde{U}$ & 0.2 & UGL & Mercury, total recoverable \\
\hline FTF 3 & $\mathrm{U}$ & 0.635 & UGL & Mercury, total recoverable \\
\hline FTF 8 & $\mathrm{U}$ & 0.2 & UGL & Mercury, total recoverable \\
\hline FTF 9 & $\mathbf{U}$ & 0.2 & UGL & Mercury, total recoverable \\
\hline FTF 11 & $\bar{U}$ & 0.2 & UGL & Mercury, total recoverable \\
\hline FTF 15 & $\mathrm{U}$ & 0.2 & UGL & Mercury, total recoverable \\
\hline FTF 19 & $\mathbf{U}$ & 0.2 & UGL & Mercury, total recoverable \\
\hline FTF 21 & $\mathrm{U}$ & 0.2 & UGL & Mercury, total recoverable \\
\hline FTF 22 & $\mathrm{U}$ & 0.2 & UGL & Mercury, total recoverable \\
\hline GBW 1 & $\mathrm{U}$ & 0.2 & UGL & Mercury, total recoverable \\
\hline HAA 1A & $\mathrm{U}$ & 0.2 & UGL & Mercury, total recoverable \\
\hline HAA 1AA & $\mathrm{U}$ & 0.2 & UGL & Mercury, total recoverable \\
\hline HAA 1B & $\mathrm{U}$ & 0.2 & UGL & Mercury, total recoverable \\
\hline HAA 2A & $\mathrm{U}$ & 0.2 & UGL & Mercury, total recoverable \\
\hline HAA 2 AA & $\mathbf{U}$ & 0.2 & UGL & Mercury, total recoverable \\
\hline HAA 2B & $\mathbf{U}$ & 0.2 & UGL & Mercury, total recoverable \\
\hline HAA 2C & $\mathbf{U}$ & 0.2 & UGL & Mercury, total recoverable \\
\hline HAA 2D & $\mathbf{U}$ & 0.2 & UGL & Mercury, total recoverable \\
\hline HAA 3A & $\mathrm{U}$ & 0.2 & UGL & Mercury, total recoverable \\
\hline HAA 3AA & $\mathrm{U}$ & 0.2 & UGL & Mercury, total recoverable \\
\hline HAA 3B & $\mathrm{U}$ & 0.2 & UGL & Mercury, total recoverable \\
\hline HAA 3C & $\mathrm{U}$ & 0.2 & UGL & Mercury, total recoverable \\
\hline HAA 4A & $\mathrm{U}$ & 0.2 & UGL & Mercury, total recoverable \\
\hline HAA 4AA & $\mathrm{U}$ & 0.2 & UGL & Mercury, total recoverable \\
\hline HAA 4B & $\mathrm{U}$ & 0.2 & UGL & Mercury, total recoverable \\
\hline HAA 4C & $\mathrm{U}$ & 0.2 & UGL & Mercury, total recoverable \\
\hline HAA 5A & $\mathrm{U}$ & 0.2 & UGL & Mercury, total recoverable \\
\hline HAA 6A & $\mathrm{U}$ & 0.2 & UGL & Mercury, total recoverable \\
\hline HAA $6 \mathrm{AA}$ & $\mathrm{U}$ & 0.2 & UGL & Mercury, total recoverable \\
\hline HAA $6 \mathrm{~B}$ & $\mathrm{U}$ & 0.2 & UGL & Mercury, total recoverable \\
\hline HAA 6C & $\mathrm{U}$ & 0.2 & UGL & Mercury, total recoverable \\
\hline HAA 6D & $\mathrm{U}$ & 0.2 & UGL & Mercury, total recoverable \\
\hline HAC 1 & $\mathrm{U}$ & 0.2 & UGL & Mercury, total recoverable \\
\hline
\end{tabular}


Wells $(n=856)$ with no estimated median mercury concentration (all values nondetect at or below nominal D.L.)

\begin{tabular}{|c|c|c|c|c|}
\hline well & qualifier & result & units & analyte name \\
\hline HAP 1 & $\mathrm{U}$ & 0.2 & UGL & Mercury, total recoverable \\
\hline HAP 2 & $\mathrm{U}$ & 0.2 & UGL & Mercury, total recoverable \\
\hline $\mathrm{HC} \quad 1 \mathrm{~A}$ & $\mathrm{U}$ & 0.2 & UGL & Mercury, total recoverable \\
\hline $\mathrm{HC} \quad 1 \mathrm{~B}$ & $\mathrm{U}$ & 0.2 & UGL & Mercury, total recoverable \\
\hline $\mathrm{HC} \quad 1 \mathrm{E}$ & $\mathrm{U}$ & 0.2 & UGL & Mercury, total recoverable \\
\hline $\mathrm{HC} \quad 2 \mathrm{~A}$ & $\mathrm{U}$ & 0.2 & UGL & Mercury, total recoverable \\
\hline $\mathrm{HC} \quad 2 \mathrm{~B}$ & $\mathrm{U}$ & 0.2 & UGL & Mercury, total recoverable \\
\hline $\mathrm{HC} 2 \mathrm{C}$ & $\mathrm{U}$ & 0.2 & UGL & Mercury, total recoverable \\
\hline $\mathrm{HC} 2 \mathrm{D}$ & $\mathrm{U}$ & 0.2 & UGL & Mercury, total recoverable \\
\hline $\mathrm{HC} \quad 2 \mathrm{E}$ & $\mathrm{U}$ & 0.2 & UGL & Mercury, total recoverable \\
\hline $\mathrm{HC} \quad 2 \mathrm{~F}$ & $\mathrm{U}$ & 0.2 & UGL & Mercury, total recoverable \\
\hline $\mathrm{HC} \quad 4 \mathrm{~A}$ & $\mathrm{U}$ & 0.625 & UGL & Mercury, total recoverable \\
\hline $\mathrm{HC} \quad 5 \mathrm{~A}$ & $\mathrm{U}$ & 0.2 & UGL & Mercury, total recoverable \\
\hline $\mathrm{HC} \quad 5 \mathrm{~B}$ & $\mathrm{U}$ & 0.2 & UGL & Mercury, total recoverable \\
\hline $\mathrm{HC} \quad 6 \mathrm{~B}$ & $\mathrm{U}$ & 0.2 & UGL & Mercury, total recoverable \\
\hline $\mathrm{HC} 10 \mathrm{~B}$ & $\mathrm{U}$ & 0.2 & UGL & Mercury, total recoverable \\
\hline $\mathrm{HC} 11 \mathrm{C}$ & $\mathrm{U}$ & 0.2 & UGL & Mercury, total recoverable \\
\hline $\mathrm{HC} \quad 12 \mathrm{~B}$ & $\mathrm{U}$ & 0.2 & UGL & Mercury, total recoverable \\
\hline $\mathrm{HCA} 1$ & $\mathrm{U}$ & 0.2 & UGL & Mercury, total recoverable \\
\hline $\mathrm{HCA} 4 \mathrm{~A}$ & $\mathrm{U}$ & 0.2 & UGL & Mercury, total recoverable \\
\hline $\mathrm{HCA} 4 \mathrm{C}$ & $\mathrm{U}$ & 0.2 & UGL & Mercury, total recoverable \\
\hline $\mathrm{HCB} 2$ & $\mathrm{U}$ & 0.2 & UGL & Mercury, total recoverable \\
\hline $\mathrm{HCB} 3$ & $\mathrm{U}$ & 0.2 & UGL & Mercury, total recoverable \\
\hline $\mathrm{HCB} 4$ & $\mathrm{U}$ & 0.2 & UGL & Mercury, total recoverable \\
\hline HET 2D & $\mathrm{U}$ & 0.2 & UGL & Mercury, total recoverable \\
\hline HHP 2D & $\mathrm{U}$ & 0.2 & UGL & Mercury, total recoverable \\
\hline HIW 2A & $\mathrm{U}$ & 0.2 & UGL & Mercury, total recoverable \\
\hline HIW 2D & $\mathrm{U}$ & 0.2 & UGL & Mercury, total recoverable \\
\hline HIW 2IC & $\mathrm{U}$ & 0.2 & UGL & Mercury, total recoverable \\
\hline HIW 2MC & $\mathrm{U}$ & 0.2 & UGL & Mercury, total recoverable \\
\hline HIW 3MC & $\mathrm{U}$ & 0.2 & UGL & Mercury, total recoverable \\
\hline HIW 4MC & $\mathrm{U}$ & 0.2 & UGL & Mercury, total recoverable \\
\hline HIW 5MC & $\mathrm{U}$ & 0.2 & UGL & Mercury, total recoverable \\
\hline HMD 1D & $\mathrm{U}$ & 0.2 & UGL & Mercury, total recoverable \\
\hline HMD 2D & $\mathbf{U}$ & 0.2 & UGL & Mercury, total recoverable \\
\hline HMD 3D & $\mathbf{U}$ & 0.2 & UGL & Mercury, total recoverable \\
\hline HMD 4D & $\mathrm{U}$ & 0.2 & UGL & Mercury, total recoverable \\
\hline HR3 14DL & $\mathrm{U}$ & 0.2 & UGL & Mercury, total recoverable \\
\hline HR3 15DL & $\mathrm{U}$ & 0.2 & UGL & Mercury, total recoverable \\
\hline HR8 12 & $\mathrm{U}$ & 0.2 & UGL & Mercury, total recoverable \\
\hline HR8 13 & $\mathrm{U}$ & 0.2 & UGL & Mercury, total recoverable \\
\hline HSB 1TB & $\mathrm{U}$ & 0.2 & UGL & Mercury, total recoverable \\
\hline HSB 69A & $\mathrm{U}$ & 0.2 & UGL & Mercury, total recoverable \\
\hline HSB 85B & $\mathrm{U}$ & 0.2 & UGL & Mercury, total recoverable \\
\hline HSB101C & $\mathrm{U}$ & 0.2 & UGL & Mercury, total recoverable \\
\hline HSB 104C & $\mathbf{U}$ & 0.2 & UGL & Mercury, total recoverable \\
\hline HSB 108C & $\mathbf{U}$ & 0.2 & UGL & Mercury, total recoverable \\
\hline HSB110D & $\mathbf{U}$ & 0.2 & UGL & Mercury, total recoverable \\
\hline HSB132C & $\mathrm{U}$ & 0.2 & UGL & Mercury, total recoverable \\
\hline HSB132D & $\mathbf{U}$ & 0.2 & UGL & Mercury, total recoverable \\
\hline HSB135C & $\mathbf{U}$ & 0.2 & UGL & Mercury, total recoverable \\
\hline HSB139A & $\mathrm{U}$ & 0.2 & UGL & Mercury, total recoverable \\
\hline
\end{tabular}




\begin{tabular}{|c|c|c|c|c|}
\hline \multicolumn{5}{|c|}{$\begin{array}{l}\text { Wells ( } \mathrm{n}=856 \text { ) with no estimated median mercury concentration (all values nondetect at or } \\
\text { below nominal D.L.) }\end{array}$} \\
\hline well & qualifier & result & units & \begin{tabular}{|c|} 
analyte_name \\
\end{tabular} \\
\hline HSB139D & $\mathrm{U}$ & 0.2 & UGL & Mercury, total recoverable \\
\hline HSB140A & $\mathrm{U}$ & 0.2 & UGL & Mercury, total recoverable \\
\hline HSB141C & $\mathrm{U}$ & 0.2 & UGL & Mercury, total recoverable \\
\hline HSB 142C & $\mathrm{U}$ & 0.2 & UGL & Mercury, total recoverable \\
\hline $\mathrm{HSB} 142 \mathrm{C}$ & $\mathrm{U}$ & 1 & UGL & Mercury, total recoverable \\
\hline HSB142D & $\mathrm{U}$ & 0.2 & UGL & Mercury, total recoverable \\
\hline HSB146D & $\mathrm{U}$ & 0.2 & UGL & Mercury, total recoverable \\
\hline HSL 6A & $\mathrm{U}$ & 0.2 & UGL & Mercury, total recoverable \\
\hline HSL 6C & $\mathrm{U}$ & 0.2 & UGL & Mercury, total recoverable \\
\hline HSL 8AA & $\mathbf{U}$ & 0.2 & UGL & Mercury, total recoverable \\
\hline HSS 1D & $\mathbf{U}$ & 0.2 & UGL & Mercury, total recoverable \\
\hline HSS 2D & $\mathbf{U}$ & 0.2 & UGL & Mercury, total recoverable \\
\hline HSS 3D & $\mathrm{U}$ & 0.2 & UGL & Mercury, total recoverable \\
\hline HTF 2 & $\bar{U}$ & 0.2 & UGL & Mercury, total recoverable \\
\hline HTF 3 & $\mathrm{U}$ & 0.2 & UGL & Mercury, total recoverable \\
\hline HTF 4 & $\mathrm{U}$ & 0.2 & UGL & Mercury, total recoverable \\
\hline HTF 5 & $\mathrm{U}$ & 0.2 & UGL & Mercury, total recoverable \\
\hline HTF 8 & $\mathrm{U}$ & 0.2 & UGL & Mercury, total recoverable \\
\hline HTF 13 & $\mathrm{U}$ & 0.2 & UGL & Mercury, total recoverable \\
\hline HTF 16 & $\mathrm{U}$ & 0.2 & UGL & Mercury, total recoverable \\
\hline HTF 17 & $\mathrm{U}$ & 0.2 & UGL & Mercury, total recoverable \\
\hline HTF 22 & $\mathrm{U}$ & 0.202 & UGL & Mercury, total recoverable \\
\hline HTF 34 & $\mathrm{U}$ & 0.2 & UGL & Mercury, total recoverable \\
\hline HWS 1A & $\mathbf{U}$ & 0.2 & UGL & Mercury, total recoverable \\
\hline HWS 2 & $\underline{U}$ & 0.2 & UGL & Mercury, total recoverable \\
\hline HXB 1 & $\mathrm{U}$ & 0.2 & UGL & Mercury, total recoverable \\
\hline $\mathrm{HXB} 2$ & $\mathrm{U}$ & 0.2 & UGL & Mercury, total recoverable \\
\hline HXB 3 & $\mathbf{U}$ & 0.2 & UGL & Mercury, total recoverable \\
\hline HXB 5D & $\mathrm{U}$ & 0.2 & UGL & Mercury, total recoverable \\
\hline IDB 1A & $\mathrm{U}$ & 0.2 & UGL & Mercury, total recoverable \\
\hline IDB 1B & $\mathrm{U}$ & 0.2 & UGL & Mercury, total recoverable \\
\hline IDB 1C & $\mathrm{U}$ & 0.2 & UGL & Mercury, total recoverable \\
\hline IDB 2B & $\mathrm{U}$ & 0.2 & UGL & Mercury, total recoverable \\
\hline IDB 2C & $\mathrm{U}$ & 0.2 & UGL & Mercury, total recoverable \\
\hline IDB 3 & $\mathrm{U}$ & 0.2 & UGL & Mercury, total recoverable \\
\hline IDB 4 & $\mathrm{U}$ & 0.2 & UGL & Mercury, total recoverable \\
\hline IDB 5 & $\mathrm{U}$ & 0.2 & UGL & Mercury, total recoverable \\
\hline IDB 6 & $\mathrm{U}$ & 0.2 & UGL & Mercury, total recoverable \\
\hline IDB 7 & $\mathrm{U}$ & 0.2 & UGL & Mercury, total recoverable \\
\hline IDB 8 & $\mathrm{U}$ & 0.2 & UGL & Mercury, total recoverable \\
\hline IDB 9 & $\mathrm{U}$ & 0.2 & UGL & Mercury, total recoverable \\
\hline IDB 10 & $\mathrm{U}$ & 0.2 & UGL & Mercury, total recoverable \\
\hline IDP 1 & $\mathrm{U}$ & 0.2 & UGL & Mercury, total recoverable \\
\hline IDP 2 & $\mathrm{U}$ & 0.2 & UGL & Mercury, total recoverable \\
\hline IDP 3A & $\mathrm{U}$ & 0.2 & UGL & Mercury, total recoverable \\
\hline IDP 3B & $\mathrm{U}$ & 0.2 & UGL & Mercury, total recoverable \\
\hline IDP 3C & $\mathrm{U}$ & 0.2 & UGL & Mercury, total recoverable \\
\hline IDP 4 & $\mathrm{U}$ & 0.2 & UGL & Mercury, total recoverable \\
\hline IDP 6 & $\mathrm{U}$ & 0.2 & UGL & Mercury, total recoverable \\
\hline IDP 7 & $\mathrm{U}$ & 0.2 & UGL & Mercury, total recoverable \\
\hline IDP 8 & $\mathrm{U}$ & 0.2 & UGL & Mercury, total recoverable \\
\hline IDP 9 & $\mathrm{U}$ & 0.2 & UGL & Mercury, total recoverable \\
\hline
\end{tabular}




\begin{tabular}{|c|c|c|c|c|}
\hline \multicolumn{5}{|c|}{$\begin{array}{l}\text { Wells }(\mathrm{n}=856) \text { with no estimated median mercury concentration (all values nondetect at or } \\
\text { below nominal D.L.) }\end{array}$} \\
\hline well & qualifier & result & units & $\begin{array}{c}\text { analyte_name } \\
\end{array}$ \\
\hline IDQ 2 & $\mathrm{U}$ & 0.2 & UGL & Mercury, total recoverable \\
\hline IDQ 3A & $\mathrm{U}$ & 0.2 & UGL & Mercury, total recoverable \\
\hline IDQ 3B & $\mathrm{U}$ & 0.2 & UGL & Mercury, total recoverable \\
\hline IDQ 3C & $\mathrm{U}$ & 0.2 & UGL & Mercury, total recoverable \\
\hline IDQ 4 & $\mathrm{U}$ & 0.2 & UGL & Mercury, total recoverable \\
\hline IDQ 5 & $\mathrm{U}$ & 0.2 & UGL & Mercury, total recoverable \\
\hline IDQ 6 & $\mathbf{U}$ & 0.2 & UGL & Mercury, total recoverable \\
\hline IDQ 7 & $\mathrm{U}$ & 0.2 & UGL & Mercury, total recoverable \\
\hline IDQ 8 & $\mathrm{U}$ & 0.2 & UGL & Mercury, total recoverable \\
\hline IDQ 9 & $\mathrm{U}$ & 0.2 & UGL & Mercury, total recoverable \\
\hline IDQ 10 & $\mathrm{U}$ & 0.2 & UGL & Mercury, total recoverable \\
\hline IDQ 12 & $\mathrm{U}$ & 0.2 & UGL & Mercury, total recoverable \\
\hline K 301P & $\mathrm{U}$ & 0.2 & UGL & Mercury, total recoverable \\
\hline KAB 1 & $\mathrm{U}$ & 0.2 & UGL & Mercury, total recoverable \\
\hline $\mathrm{KAB} 2$ & $\mathrm{U}$ & 0.2 & UGL & Mercury, total recoverable \\
\hline KAB 3 & $\mathrm{U}$ & 0.2 & UGL & Mercury, total recoverable \\
\hline $\mathrm{KAB} 4$ & $\mathrm{U}$ & 0.2 & UGL & Mercury, total recoverable \\
\hline KAC 2 & $\mathrm{U}$ & 0.2 & UGL & Mercury, total recoverable \\
\hline $\mathrm{KAC} 6$ & $\mathrm{U}$ & 0.2 & UGL & Mercury, total recoverable \\
\hline $\mathrm{KAC} 7$ & $\mathrm{U}$ & 0.2 & UGL & Mercury, total recoverable \\
\hline $\mathrm{KCB} 1$ & $U$ & 0.2 & UGL & Mercury, total recoverable \\
\hline KCB 2 & $\mathrm{U}$ & 0.2 & UGL & Mercury, total recoverable \\
\hline KDB 1 & $\mathbf{U}$ & 0.2 & UGL & Mercury, total recoverable \\
\hline $\mathrm{KDB} 2$ & $\mathrm{U}$ & 0.2 & UGL & Mercury, total recoverable \\
\hline KDB 3 & $\mathrm{U}$ & 0.2 & UGL & Mercury, total recoverable \\
\hline KDT 1D & $\mathrm{U}$ & 0.2 & UGL & Mercury, total recoverable \\
\hline KRB 16D & $\mathbf{U}$ & 0.2 & UGL & Mercury, total recoverable \\
\hline KRB 17D & $\mathrm{U}$ & 0.2 & UGL & Mercury, total recoverable \\
\hline KRB 18D & $\mathbf{U}$ & 0.2 & UGL & Mercury, total recoverable \\
\hline KRB 19D & $\mathbf{U}$ & 0.2 & UGL & Mercury, total recoverable \\
\hline KRP 1 & $\mathrm{U}$ & 0.2 & UGL & Mercury, total recoverable \\
\hline KRP 2 & $\mathrm{U}$ & 0.2 & UGL & Mercury, total recoverable \\
\hline KRP 3 & $\mathrm{U}$ & 0.2 & UGL & Mercury, total recoverable \\
\hline KRP 4 & $\mathrm{U}$ & 0.2 & UGL & Mercury, total recoverable \\
\hline KRP 8 & $\mathrm{U}$ & 0.2 & UGL & Mercury, total recoverable \\
\hline KRP 9 & $\mathrm{U}$ & 0.2 & UGL & Mercury, total recoverable \\
\hline KSB 1 & $\mathrm{U}$ & 0.2 & UGL & Mercury, total recoverable \\
\hline KSB 2 & $\mathrm{U}$ & 0.2 & UGL & Mercury, total recoverable \\
\hline KSB 3 & $\mathrm{U}$ & 0.2 & UGL & Mercury, total recoverable \\
\hline KSB 4A & $\mathrm{U}$ & 0.2 & UGL & Mercury, total recoverable \\
\hline KSM 1D & $\mathrm{U}$ & 0.2 & UGL & Mercury, total recoverable \\
\hline KSS 1D & $\mathrm{U}$ & 0.2 & UGL & Mercury, total recoverable \\
\hline KSS 2D & $\mathrm{U}$ & 0.2 & UGL & Mercury, total recoverable \\
\hline KSS 3D & $\mathrm{U}$ & 0.2 & UGL & Mercury, total recoverable \\
\hline LAC 6DL & $\mathrm{U}$ & 0.128 & UGL & Mercury, total recoverable \\
\hline LAC 7DU & $\mathrm{U}$ & 0.2 & UGL & Mercury, total recoverable \\
\hline LAC 8DL & $\mathrm{U}$ & 0.2 & UGL & Mercury, total recoverable \\
\hline LAW 1C & $\mathrm{U}$ & 0.2 & UGL & Mercury, total recoverable \\
\hline LAW 1D & $\mathrm{U}$ & 0.067 & UGL & Mercury, total recoverable \\
\hline LAW 1F & $\mathrm{U}$ & 0.2 & UGL & Mercury, total recoverable \\
\hline LAW 3B & $\mathrm{U}$ & 0.2 & UGL & Mercury, total recoverable \\
\hline LAW 3C & $\mathrm{U}$ & 0.2 & UGL & Mercury, total recoverable \\
\hline
\end{tabular}


Wells $(n=856)$ with no estimated median mercury concentration (all values nondetect at or below nominal D.L.)

\begin{tabular}{|c|c|c|c|c|}
\hline well & qualifier & result & units & analyte_name \\
\hline LBP 1D & $\mathrm{U}$ & 0.2 & UGL & Mercury, total recoverable \\
\hline $\mathrm{LCO} 1$ & $\mathrm{U}$ & 0.2 & UGL & Mercury, total recoverable \\
\hline LCO 3 & $\mathrm{U}$ & 0.2 & UGL & Mercury, total recoverable \\
\hline $\mathrm{LCO} 5 \mathrm{~A}$ & $\mathrm{U}$ & 0.2 & UGL & Mercury, total recoverable \\
\hline $\mathrm{LCO} 5 \mathrm{C}$ & $\mathrm{U}$ & 0.2 & UGL & Mercury, total recoverable \\
\hline LCO 5DL & $\mathrm{U}$ & 0.2 & UGL & Mercury, total recoverable \\
\hline $\mathrm{LCO}$ 6DL & $\mathrm{U}$ & 0.2 & UGL & Mercury, total recoverable \\
\hline LCO 7DL & $\mathrm{U}$ & 0.2 & UGL & Mercury, total recoverable \\
\hline LCO 8DL & $\mathrm{U}$ & 0.2 & UGL & Mercury, total recoverable \\
\hline LDB 1 & $\bar{U}$ & 0.2 & $\overline{\mathrm{UGL}}$ & Mercury, total recoverable \\
\hline LDB 2 & $\mathrm{U}$ & 0.2 & UGL & Mercury, total recoverable \\
\hline LDB 3 & $\mathrm{U}$ & 0.2 & UGL & Mercury, total recoverable \\
\hline LFW 6 & $\mathrm{U}$ & 0.2 & UGL & Mercury, total recoverable \\
\hline LFW 7 & $\mathrm{U}$ & 0.2 & $\mathrm{UGL}$ & Mercury, total recoverable \\
\hline LFW 8 & $\mathrm{U}$ & 0.2 & $\mathrm{UGL}$ & Mercury, total recoverable \\
\hline LFW 19 & $\mathrm{U}$ & 0.2 & UGL & Mercury, total recoverable \\
\hline LFW 20 & $\mathrm{U}$ & 0.2 & UGL & Mercury, total recoverable \\
\hline LFW 25 & $\mathrm{U}$ & 0.2 & UGL & Mercury, total recoverable \\
\hline LFW 26 & $\mathrm{U}$ & 0.2 & UGL & Mercury, total recoverable \\
\hline LFW 27 & $\mathrm{U}$ & 0.2 & UGL & Mercury, total recoverable \\
\hline LFW $30^{\circ}$ & $\bar{U}$ & 0.2 & UGL & Mercury, total recoverable \\
\hline LFW 33 & $\mathrm{U}$ & 0.2 & UGL & Mercury, total recoverable \\
\hline LFW 34 & $U$ & 0.2 & UGL & Mercury, total recoverable \\
\hline LFW 35 & $\mathrm{U}$ & 0.2 & UGL & Mercury, total recoverable \\
\hline LFW 36 & $\mathrm{U}$ & 0.2 & UGL & Mercury, total recoverable \\
\hline LFW 37 & $\bar{U}$ & 0.2 & UGL & Mercury, total recoverable \\
\hline LFW 40 & $\mathrm{U}$ & 0.2 & UGL & Mercury, total recoverable \\
\hline LFW 41 & $\mathrm{U}$ & 0.2 & UGL & Mercury, total recoverable \\
\hline LFW 41R & $\mathrm{U}$ & 0.2 & UGL & Mercury, total recoverable \\
\hline LFW 42 & $\mathrm{U}$ & 0.2 & UGL & Mercury, total recoverable \\
\hline LFW 44D & $\overline{\mathrm{U}}$ & 0.2 & UGL & Mercury, total recoverable \\
\hline LFW 46D & $\underline{U}$ & 0.2 & UGL & Mercury, total recoverable \\
\hline LFW 48D & $\mathbf{U}$ & 0.2 & UGL & Mercury, total recoverable \\
\hline LFW 55D & $\bar{U}$ & 0.2 & UGL & Mercury, total recoverable \\
\hline LFW 57B & $\mathrm{U}$ & 0.2 & UGL & Mercury, total recoverable \\
\hline LFW 57C & $\overline{\mathrm{U}}$ & 0.2 & UGL & Mercury, total recoverable \\
\hline LFW 59B & $\mathrm{U}$ & 0.2 & UGL & Mercury, total recoverable \\
\hline LFW 59C & $\mathrm{U}$ & 0.2 & UGL & Mercury, total recoverable \\
\hline LFW 60B & $\mathrm{U}$ & 0.2 & UGL & Mercury, total recoverable \\
\hline LFW 61C & $\underline{U}$ & 0.2 & UGL & Mercury, total recoverable \\
\hline LFW 62B & $\mathrm{U}$ & 0.2 & UGL & Mercury, total recoverable \\
\hline LFW 64B & $\bar{U}$ & 0.2 & UGL & Mercury, total recoverable \\
\hline LFW 65C & $\mathbf{U}$ & 0.2 & UGL & Mercury, total recoverable \\
\hline LFW 66B & $\mathbf{U}$ & 0.2 & UGL & Mercury, total recoverable \\
\hline LFW 67B & $\mathrm{U}$ & 0.2 & UGL & Mercury, total recoverable \\
\hline LFW 68B & $\mathbf{U}$ & 0.2 & UGL & Mercury, total recoverable \\
\hline LFW 68C & $\mathbf{U}$ & 0.2 & UGL & Mercury, total recoverable \\
\hline LFW 69B & $\mathrm{U}$ & 0.2 & UGL & Mercury, total recoverable \\
\hline LFW 70C & $\mathbf{U}$ & 0.2 & UGL & Mercury, total recoverable \\
\hline LFW 70D & $\mathrm{U}$ & 0.2 & UGL & Mercury, total recoverable \\
\hline LFW 71C & $\mathrm{U}$ & 0.2 & UGL & Mercury, total recoverable \\
\hline LFW 72D & $\mathrm{U}$ & 0.2 & UGL & Mercury, total recoverable \\
\hline
\end{tabular}




\begin{tabular}{|c|c|c|c|c|}
\hline \multicolumn{5}{|c|}{ Wells ( $n=856$ ) with no estimated median mercury concentration (all values nondetect at or } \\
\hline well & qualifier & result & units & analyte name \\
\hline LRP 1 & $\mathrm{U}$ & 0.2 & UGL & Mercury, total recoverable \\
\hline LRP 2 & $\bar{U}$ & 0.2 & UGL & Mercury, total recoverable \\
\hline LRP 3 & $\mathrm{U}$ & 0.2 & UGL & Mercury, total recoverable \\
\hline LSB 1 & $\mathrm{U}$ & 0.2 & UGL & Mercury, total recoverable \\
\hline LSB 2 & $\bar{U}$ & $\overline{0.2}$ & UGL & Mercury, total recoverable \\
\hline LSB 3 & $\mathrm{U}$ & 0.2 & UGL & Mercury, total recoverable \\
\hline LSB 4 & $\mathrm{U}$ & 0.2 & UGL & Mercury, total recoverable \\
\hline MCB 2 & $\mathrm{U}$ & 0.2 & UGL & Mercury, total recoverable \\
\hline $\mathrm{MCB} 4$ & $\mathrm{U}$ & 0.2 & UGL & Mercury, total recoverable \\
\hline MCB $7 \mathrm{C}$ & $\mathrm{U}$ & 0.2 & UGL & Mercury, total recoverable \\
\hline MCB 11D & $\mathrm{U}$ & 0.2 & UGL & Mercury, total recoverable \\
\hline MCB 13C & $\mathrm{U}$ & 0.2 & UGL & Mercury, total recoverable \\
\hline MCB 14C & $\mathrm{U}$ & 0.2 & UGL & Mercury, total recoverable \\
\hline MCB 14C & $\mathrm{U}$ & 0.2 & UGL & Mercury, total recoverable \\
\hline MCB 15B & $\mathrm{U}$ & 0.2 & UGL & Mercury, total recoverable \\
\hline MCB 15C & $\mathrm{U}$ & 0.2 & UGL & Mercury, total recoverable \\
\hline MCB 16B & $\mathbf{U}$ & 0.2 & UGL & Mercury, total recoverable \\
\hline MCB 16C & $\mathrm{U}$ & 0.2 & UGL & Mercury, total recoverable \\
\hline $\mathrm{MCB} 17 \mathrm{~B}$ & $\bar{U}$ & 0.2 & UGL & Mercury, total recoverable \\
\hline MCB 18B & $\mathrm{U}$ & 0.2 & UGL & Mercury, total recoverable \\
\hline MCB 19B & $\mathrm{U}$ & 0.2 & UGL & Mercury, total recoverable \\
\hline MGC 36 & $\mathrm{U}$ & 0.2 & UGL & Mercury, total recoverable \\
\hline MSB 1B & $\mathrm{U}$ & 0.2 & UGL & Mercury, total recoverable \\
\hline MSB 1C & $\mathrm{U}$ & 0.2 & UGL & Mercury, total recoverable \\
\hline MSB 1D & $\mathrm{U}$ & 0.2 & UGL & Mercury, total recoverable \\
\hline MSB 2C & $\mathrm{U}$ & 0.2 & UGL & Mercury, total recoverable \\
\hline MSB 2D & $\mathrm{U}$ & 0.2 & UGL & Mercury, total recoverable \\
\hline MSB 3C & $\bar{U}$ & 0.2 & UGL & Mercury, total recoverable \\
\hline MSB 3D & $\mathrm{U}$ & 0.2 & UGL & Mercury, total recoverable \\
\hline MSB 4C & $\mathrm{U}$ & 0.2 & UGL & Mercury, total recoverable \\
\hline MSB 5B & $\mathrm{U}$ & 0.2 & UGL & Mercury, total recoverable \\
\hline MSB 6A & $\mathrm{U}$ & 0.2 & UGL & Mercury, total recoverable \\
\hline MSB 6C & $\mathrm{U}$ & 0.2 & UGL & Mercury, total recoverable \\
\hline MSB 7B & $\mathrm{U}$ & 0.2 & UGL & Mercury, total recoverable \\
\hline MSB 9A & $\mathbf{U}$ & 0.2 & UGL & Mercury, total recoverable \\
\hline MSB 9B & $\mathrm{U}$ & 0.2 & UGL & Mercury, total recoverable \\
\hline MSB 10A & $\mathrm{U}$ & 0.2 & UGL & Mercury, total recoverable \\
\hline MSB 10C & $\mathrm{U}$ & 0.2 & UGL & Mercury, total recoverable \\
\hline MSB 11A & $\bar{U}$ & 0.2 & UGL & Mercury, total recoverable \\
\hline MSB 11B & $\mathrm{U}$ & 0.2 & UGL & Mercury, total recoverable \\
\hline MSB 11D & $\mathrm{U}$ & 0.2 & UGL & Mercury, total recoverable \\
\hline MSB 11F & $\mathrm{U}$ & 0.2 & UGL & Mercury, total recoverable \\
\hline MSB 12A & $\mathrm{U}$ & 0.2 & UGL & Mercury, total recoverable \\
\hline MSB 12C & $\mathrm{U}$ & 0.219 & UGL & Mercury, total recoverable \\
\hline MSB 12TA & $\mathrm{U}$ & 0.2 & UGL & Mercury, total recoverable \\
\hline MSB $12 \mathrm{~TB}$ & $\mathrm{U}$ & 0.2 & UGL & Mercury, total recoverable \\
\hline MSB 13A & $\mathrm{U}$ & 0.2 & UGL & Mercury, total recoverable \\
\hline MSB 13B & $\mathrm{U}$ & 0.2 & UGL & Mercury, total recoverable \\
\hline MSB 13D & $\mathbf{U}$ & 0.2 & UGL & Mercury, total recoverable \\
\hline MSB 14C & $\bar{U}$ & 0.2 & UGL & Mercury, total recoverable \\
\hline MSB 15AA & $\mathbf{U}$ & 0.2 & UGL & Mercury, total recoverable \\
\hline MSB 16A & $\mathrm{U}$ & 0.2 & UGL & Mercury, total recoverable \\
\hline
\end{tabular}


$\overline{\text { Wells ( } \mathrm{n}=856 \text { ) with no estimated median mercury concentration (all values nondetect at or }}$ below nominal D.L.)

\begin{tabular}{|c|c|c|c|c|}
\hline well & qualifier & result & units & analyte name \\
\hline MSB 16C & $\mathrm{U}$ & 0.2 & UGL & Mercury, total recoverable \\
\hline MSB 17BB & $\mathrm{U}$ & 0.2 & UGL & Mercury, total recoverable \\
\hline MSB 17D & $\mathrm{U}$ & 0.2 & UGL & Mercury, total recoverable \\
\hline MSB 18A & $\mathrm{U}$ & 0.2 & UGL & Mercury, total recoverable \\
\hline MSB 18B & $\mathrm{U}$ & 0.2 & UGL & Mercury, total recoverable \\
\hline MSB 18C & $\mathrm{U}$ & 0.2 & UGL & Mercury, total recoverable \\
\hline MSB 19A & $\mathrm{U}$ & 0.2 & UGL & Mercury, total recoverable \\
\hline MSB 19B & $\mathrm{U}$ & 0.2 & UGL & Mercury, total recoverable \\
\hline MSB 20C & $\mathrm{U}$ & 0.2 & UGL & Mercury, total recoverable \\
\hline MSB 21B & $\mathrm{U}$ & 0.2 & UGL & Mercury, total recoverable \\
\hline MSB 21C & $\mathrm{U}$ & 0.2 & UGL & Mercury, total recoverable \\
\hline MSB 21TA & $\mathbf{U}$ & 0.2 & UGL & Mercury, total recoverable \\
\hline MSB 23TR & $\mathrm{U}$ & 0.2 & UGL & Mercury, total recoverable \\
\hline MSB 26 & $\mathrm{U}$ & 0.2 & UGL & Mercury, total recoverable \\
\hline MSB 26B & $\mathrm{U}$ & 0.2 & UGL & Mercury, total recoverable \\
\hline MSB 27A & $\mathrm{U}$ & 0.2 & UGL & Mercury, total recoverable \\
\hline MSB 27B & $\mathrm{U}$ & 0.2 & UGL & Mercury, total recoverable \\
\hline MSB 28 & $\mathrm{U}$ & 0.2 & UGL & Mercury, total recoverable \\
\hline MSB 29A & $\mathrm{U}$ & 0.2 & UGL & Mercury, total recoverable \\
\hline MSB 29TA & $U$ & 0.2 & UGL & Mercury, total recoverable \\
\hline MSB 30A & $\mathrm{U}$ & 0.2 & UGL & Mercury, total recoverable \\
\hline MSB 30AA & $\mathbf{U}$ & 0.2 & UGL & Mercury, total recoverable \\
\hline MSB 30B & $\mathbf{U}$ & 0.2 & UGL & Mercury, total recoverable \\
\hline MSB 30CC & $\mathrm{U}$ & 0.2 & UGL & Mercury, total recoverable \\
\hline MSB 31A & $\mathrm{U}$ & 0.2 & UGL & Mercury, total recoverable \\
\hline MSB 32 & $\mathrm{U}$ & 0.2 & UGL & Mercury, total recoverable \\
\hline MSB 32C & $\mathrm{U}$ & 0.2 & UGL & Mercury, total recoverable \\
\hline MSB 33 & $\mathrm{U}$ & 0.2 & UGL & Mercury, total recoverable \\
\hline MSB 33A & $\mathrm{U}$ & 0.2 & UGL & Mercury, total recoverable \\
\hline MSB 33B & $\mathrm{U}$ & 0.2 & UGL & Mercury, total recoverable \\
\hline MSB 33TA & $\mathrm{U}$ & 0.2 & UGL & Mercury, total recoverable \\
\hline MSB 34A & $\mathbf{U}$ & 0.2 & UGL & Mercury, total recoverable \\
\hline MSB 35A & $\mathrm{U}$ & 0.2 & UGL & Mercury, total recoverable \\
\hline MSB 35B & $\mathrm{U}$ & 0.2 & UGL & Mercury, total recoverable \\
\hline MSB 35TA & $\mathbf{U}$ & 0.2 & UGL & Mercury, total recoverable \\
\hline MSB 37A & $\bar{U}$ & 0.2 & UGL & Mercury, total recoverable \\
\hline MSB 37D & $\mathrm{U}$ & 0.2 & UGL & Mercury, total recoverable \\
\hline MSB 38D & $\mathbf{U}$ & 0.2 & UGL & Mercury, total recoverable \\
\hline MSB 38TA & $U$ & 0.2 & UGL & Mercury, total recoverable \\
\hline MSB 39A & $\mathrm{U}$ & 0.2 & UGL & Mercury, total recoverable \\
\hline MSB 39TA & $\mathrm{U}$ & 0.2 & UGL & Mercury, total recoverable \\
\hline MSB 40A & $\mathrm{U}$ & 0.2 & UGL & Mercury, total recoverable \\
\hline MSB 40C & $\mathbf{U}$ & 0.2 & UGL & Mercury, total recoverable \\
\hline MSB 40D & $\mathrm{U}$ & 0.2 & UGL & Mercury, total recoverable \\
\hline MSB 40TA & $\mathrm{U}$ & 0.2 & UGL & Mercury, total recoverable \\
\hline MSB 41A & $\mathbf{U}$ & 0.2 & UGL & Mercury, total recoverable \\
\hline MSB 41C & $\mathbf{U}$ & 0.2 & UGL & Mercury, total recoverable \\
\hline MSB 41D & $\mathbf{U}$ & 0.2 & UGL & Mercury, total recoverable \\
\hline MSB 42A & $\mathrm{U}$ & 0.2 & UGL & Mercury, total recoverable \\
\hline MSB 42TA & $\mathrm{U}$ & 0.2 & UGL & Mercury, total recoverable \\
\hline MSB 43A & $\mathrm{U}$ & 0.2 & UGL & Mercury, total recoverable \\
\hline MSB 44A & $\mathrm{U}$ & 0.2 & UGL & Mercury, total recoverable \\
\hline
\end{tabular}


Wells ( $n=856$ ) with no estimated median mercury concentration (all values nondetect at or below nominal D.L.)

\begin{tabular}{|c|c|c|c|c|}
\hline well & qualifier & result & units & analyte_name \\
\hline MSB 44B & $\mathrm{U}$ & 0.2 & UGL & Mercury, total recoverable \\
\hline MSB 46B & U & 0.2 & UGL & Mercury, total recoverable \\
\hline MSB 46C & $\mathrm{U}$ & 0.2 & UGL & Mercury, total recoverable \\
\hline MSB 47BB & $\mathrm{U}$ & 0.2 & UGL & Mercury, total recoverable \\
\hline MSB 48D & $\mathrm{U}$ & 0.2 & UGL & Mercury, total recoverable \\
\hline MSB 48TA & $\bar{U}$ & 0.2 & UGL & Mercury, total recoverable \\
\hline MSB 49A & $\mathrm{U}$ & 0.2 & UGL & Mercury, total recoverable \\
\hline MSB 50B & $\mathrm{U}$ & 0.2 & UGL & Mercury, total recoverable \\
\hline MSB 50D & $\mathrm{U}$ & 0.2 & UGL & Mercury, total recoverable \\
\hline MSB 51D & $\mathrm{U}$ & 0.2 & UGL & Mercury, total recoverable \\
\hline MSB 52B & $\mathrm{U}$ & 0.2 & UGL & Mercury, total recoverable \\
\hline MSB 52D & $\mathrm{U}$ & 0.2 & UGL & Mercury, total recoverable \\
\hline MSB 53B & $\mathrm{U}$ & 0.2 & UGL & Mercury, total recoverable \\
\hline MSB 53C & $\mathrm{U}$ & 0.2 & UGL & Mercury, total recoverable \\
\hline MSB 53D & $\mathrm{U}$ & 0.2 & UGL & Mercury, total recoverable \\
\hline MSB 54B & $\mathbf{U}$ & 0.2 & UGL & Mercury, total recoverable \\
\hline MSB 54C & $\mathbf{U}$ & 0.2 & UGL & Mercury, total recoverable \\
\hline MSB 54D & $\mathrm{U}$ & 0.2 & UGL & Mercury, total recoverable \\
\hline MSB 54TA & $\mathrm{U}$ & 0.2 & UGL & Mercury, total recoverable \\
\hline MSB 55B & $\mathrm{U}$ & 0.2 & UGL & Mercury, total recoverable \\
\hline MSB 55HC & $\mathrm{U}$ & 0.2 & UGL & Mercury, total recoverable \\
\hline MSB 55TA & $\mathrm{U}$ & 0.2 & UGL & Mercury, total recoverable \\
\hline MSB 61C & $\mathrm{U}$ & 0.2 & UGL & Mercury, total recoverable \\
\hline MSB 61D & $\mathrm{U}$ & 0.2 & UGL & Mercury, total recoverable \\
\hline MSB 64B & $\mathrm{U}$ & 0.2 & UGL & Mercury, total recoverable \\
\hline MSB 66TA & $\mathrm{U}$ & 0.2 & UGL & Mercury, total recoverable \\
\hline MSB 67B & $\mathrm{U}$ & 0.2 & UGL & Mercury, total recoverable \\
\hline MSB 67D & $\mathrm{U}$ & 0.2 & UGL & Mercury, total recoverable \\
\hline MSB $68 \mathrm{C}$ & $\mathrm{U}$ & 0.2 & UGL & Mercury, total recoverable \\
\hline MSB 68D & $\mathrm{U}$ & 0.2 & UGL & Mercury, total recoverable \\
\hline MSB 69B & $\mathrm{U}$ & 0.2 & UGL & Mercury, total recoverable \\
\hline MSB 69C & $\mathrm{U}$ & 0.2 & UGL & Mercury, total recoverable \\
\hline MSB 69D & U & 0.2 & UGL & Mercury, total recoverable \\
\hline MSB 69TA & $\mathrm{U}$ & 0.2 & UGL & Mercury, total recoverable \\
\hline MSB 70D & $\mathrm{U}$ & 0.2 & UGL & Mercury, total recoverable \\
\hline MSB 71B & $\mathrm{U}$ & 0.2 & UGL & Mercury, total recoverable \\
\hline MSB 73B & $\mathrm{U}$ & 0.2 & UGL & Mercury, total recoverable \\
\hline MSB 74B & $\mathrm{U}$ & 0.09 & UGL & Mercury, total recoverable \\
\hline MSB 74C & $\mathbf{U}$ & 0.2 & UGL & Mercury, total recoverable \\
\hline MSB 74D & $\mathrm{U}$ & 0.2 & UGL & Mercury, total recoverable \\
\hline MSB 75B & $\mathrm{U}$ & 0.2 & UGL & Mercury, total recoverable \\
\hline MSB 75C & $\mathrm{U}$ & 0.2 & UGL & Mercury, total recoverable \\
\hline MSB 77B & $\mathrm{U}$ & 0.2 & UGL & Mercury, total recoverable \\
\hline MSB 77C & $\mathrm{U}$ & 0.2 & UGL & Mercury, total recoverable \\
\hline MSB 77D & $\underline{\mathrm{U}}$ & 0.2 & UGL & Mercury, total recoverable \\
\hline MSB 77'TA & $\mathrm{U}$ & 0.2 & UGL & Mercury, total recoverable \\
\hline MSB 78DR & $\mathrm{U}$ & 0.2 & UGL & Mercury, total recoverable \\
\hline MSB 79B & $\mathrm{U}$ & 0.2 & UGL & Mercury, total recoverable \\
\hline MSB 79C & $\mathrm{U}$ & 0.073 & UGL & Mercury, total recoverable \\
\hline MSB 82D & $\mathrm{U}$ & 0.2 & UGL & Mercury, total recoverable \\
\hline MSB 82TA & $\mathrm{U}$ & 0.2 & UGL & Mercury, total recoverable \\
\hline MSB 83B & $\mathrm{U}$ & 0.2 & UGL & Mercury, total recoverable \\
\hline
\end{tabular}




\begin{tabular}{|c|c|c|c|c|}
\hline \multicolumn{5}{|c|}{$\begin{array}{l}\text { Wells ( } \mathrm{n}=856) \text { with no estimated median mercury concentration (all values nondetect at or } \\
\text { below nominal D.L.) }\end{array}$} \\
\hline well & qualifier & result & units & analyte_name \\
\hline MSB 83C & $\mathrm{U}$ & 0.2 & UGL & Mercury, total recoverable \\
\hline MSB 83D & $\bar{U}$ & 0.2 & UGL & Mercury, total recoverable \\
\hline MSB 83TA & $\mathrm{U}$ & 0.2 & UGL & Mercury, total recoverable \\
\hline MSB 84C & $\mathrm{U}$ & 0.2 & UGL & Mercury, total recoverable \\
\hline MSB 85B & $\bar{U}$ & 0.2 & UGL & Mercury, total recoverable \\
\hline MSB 85C & $\mathrm{U}$ & 0.2 & UGL & Mercury, total recoverable \\
\hline MSB 85D & $\mathbf{U}$ & 0.2 & UGL & Mercury, total recoverable \\
\hline MSB 85TA & $\mathrm{U}$ & 0.2 & UGL & Mercury, total recoverable \\
\hline MSB 87B & $\mathrm{U}$ & 0.2 & UGL & Mercury, total recoverable \\
\hline MSB 87C & $\mathrm{U}$ & 0.2 & UGL & Mercury, total recoverable \\
\hline MSB 88D & $\mathrm{U}$ & 0.2 & UGL & Mercury, total recoverable \\
\hline MSB 89B & $\mathrm{U}$ & 0.2 & UGL & Mercury, total recoverable \\
\hline NBG 3 & $\mathrm{U}$ & 0.2 & UGL & Mercury, total recoverable \\
\hline NBG 4 & $\bar{U}$ & 0.2 & $\overline{\mathrm{UGL}}$ & Mercury, total recoverable \\
\hline NPM 2 & $\mathrm{U}$ & 0.2 & UGL & Mercury, total recoverable \\
\hline NPM 3 & $\mathrm{U}$ & 0.2 & UGL & Mercury, total recoverable \\
\hline NPM 4 & $U$ & 0.2 & UGL & Mercury, total recoverable \\
\hline NPM 4DD & $\mathrm{U}$ & 0.2 & UGL & Mercury, total recoverable \\
\hline NPM 19A & $\bar{U}$ & 0.2 & UGL & Mercury, total recoverable \\
\hline NPM 19B & $\bar{U}$ & 0.2 & UGL & Mercury, total recoverable \\
\hline NPM 19B & $\mathrm{U}$ & 0.2 & UGL & Mercury, total recoverable \\
\hline NPM 19B & $\mathrm{U}$ & 0.2 & UGL & Mercury, total recoverable \\
\hline NPM 19B & $\mathrm{U}$ & 0.2 & UGL & Mercury, total recoverable \\
\hline NPM 19B & $\mathrm{U}$ & 0.2 & UGL & Mercury, total recoverable \\
\hline NPM 19C & $\mathrm{U}$ & 0.2 & UGL & Mercury, total recoverable \\
\hline NPM 19D & $\mathrm{U}$ & 0.2 & UGL & Mercury, total recoverable \\
\hline NPM 19E & $\overline{\mathrm{U}}$ & 0.2 & UGL & Mercury, total recoverable \\
\hline NPM 34A & $\mathrm{U}$ & 0.2 & UGL & Mercury, total recoverable \\
\hline NPM 34B & $\mathrm{U}$ & 0.2 & UGL & Mercury, total recoverable \\
\hline NPM 34C & $\mathrm{U}$ & 0.2 & UGL & Mercury, total recoverable \\
\hline NPM 34D & $\mathrm{U}$ & 0.2 & UGL & Mercury, total recoverable \\
\hline NPM 34E & $\mathrm{U}$ & 0.2 & UGL & Mercury, total recoverable \\
\hline $\mathrm{P} 20 \mathrm{C}$ & $\mathrm{U}$ & 0.2 & UGL & Mercury, total recoverable \\
\hline $\mathrm{P} 20 \mathrm{D}$ & $\overline{\mathrm{U}}$ & 0.2 & UGL & Mercury, total recoverable \\
\hline $\mathrm{P} 24 \mathrm{~A}$ & $\mathrm{U}$ & 0.2 & UGL & Mercury, total recoverable \\
\hline P 26B & $\mathrm{U}$ & 0.2 & UGL & Mercury, total recoverable \\
\hline $\mathrm{P} \quad 27 \mathrm{~B}$ & $\mathrm{U}$ & 0.2 & UGL & Mercury, total recoverable \\
\hline $\mathrm{P} 27 \mathrm{C}$ & $\underline{\mathrm{U}}$ & 0.2 & UGL & Mercury, total recoverable \\
\hline $\mathrm{P} 27 \mathrm{D}$ & $\mathrm{U}$ & 0.2 & UGL & Mercury, total recoverable \\
\hline $\mathrm{P} \quad 27 \mathrm{~TB}$ & $\mathrm{U}$ & 0.2 & UGL & Mercury, total recoverable \\
\hline $\mathrm{P} \quad 27 \mathrm{TC}$ & $\mathrm{U}$ & 0.2 & UGL & Mercury, total recoverable \\
\hline $\begin{array}{l}\mathrm{P} \quad 27 \mathrm{TD} \\
\end{array}$ & $U$ & 0.2 & UGL & Mercury, total recoverable \\
\hline $\mathrm{P} \quad 27 \mathrm{TE}$ & $\mathrm{U}$ & 0.2 & UGL & Mercury, total recoverable \\
\hline $\mathrm{P} 28 \mathrm{~A}$ & $\mathrm{U}$ & 0.2 & UGL & Mercury, total recoverable \\
\hline $\mathrm{P} 28 \mathrm{~TB}$ & $\mathrm{U}$ & 0.2 & UGL & Mercury, total recoverable \\
\hline $\mathrm{P} 29 \mathrm{C}$ & $\mathrm{U}$ & 0.2 & UGL & Mercury, total recoverable \\
\hline $\mathrm{P} 29 \mathrm{D}$ & $\mathrm{U}$ & 0.2 & UGL & Mercury, total recoverable \\
\hline P 29TA & $\mathrm{U}$ & 0.2 & UGL & Mercury, total recoverable \\
\hline P 29TC & $\mathrm{U}$ & 0.2 & UGL & Mercury, total recoverable \\
\hline $\mathrm{P} 80$ & $\bar{U}$ & 0.2 & UGL & Mercury, total recoverable \\
\hline PAC 2 & $\mathrm{U}$ & 0.2 & UGL & Mercury, total recoverable \\
\hline PAC 3 & $\mathrm{U}$ & 0.2 & UGL & Mercury, total recoverable \\
\hline
\end{tabular}


$\overline{\text { Wells }(\mathrm{n}=856) \text { with no estimated median mercury concentration (all values nondetect at or }}$ below nominal D.L.)

\begin{tabular}{|c|c|c|c|c|}
\hline well & qualifier & result & units & analyte_name \\
\hline PAC 5 & $\mathrm{U}$ & 0.2 & UGL & Mercury, total recoverable \\
\hline PAC 6 & U & 0.2 & $\mathrm{UGL}$ & Mercury, total recoverable \\
\hline PB 1 & $\mathrm{U}$ & 0.2 & UGL & Mercury, total recoverable \\
\hline PBP 1D & $\mathrm{U}$ & 0.2 & UGL & Mercury, total recoverable \\
\hline PBP 3D & $\mathrm{U}$ & 0.2 & UGL & Mercury, total recoverable \\
\hline PCB 1A & $\mathrm{U}$ & 0.2 & UGL & Mercury, total recoverable \\
\hline PCB 3A & $\mathrm{U}$ & 0.2 & UGL & Mercury, total recoverable \\
\hline PDB 2 & $\mathrm{U}$ & 0.2 & UGL & Mercury, total recoverable \\
\hline PDB 3 & $\mathrm{U}$ & 0.2 & UGL & Mercury, total recoverable \\
\hline PDB 4 & $\overline{\mathrm{U}}$ & 0.2 & UGL & Mercury, total recoverable \\
\hline PDB 5 & $\mathrm{U}$ & 0.2 & UGL & Mercury, total recoverable \\
\hline PRP 1A & $\mathrm{U}$ & 0.2 & UGL & Mercury, total recoverable \\
\hline PRP 5 & $\mathrm{U}$ & 0.2 & UGL & Mercury, total recoverable \\
\hline PSB 1A & $\mathbf{U}$ & 0.2 & UGL & Mercury, total recoverable \\
\hline PSB 2A & $\mathbf{U}$ & 0.2 & UGL & Mercury, total recoverable \\
\hline PSB 3A & $\mathbf{U}$ & 0.2 & UGL & Mercury, total recoverable \\
\hline PSB 4A & $\mathrm{U}$ & 0.2 & UGL & Mercury, total recoverable \\
\hline PSB 5A & $\mathbf{U}$ & 0.2 & UGL & Mercury, total recoverable \\
\hline PSB 6A & $\mathrm{U}$ & 0.2 & UGL & Mercury, total recoverable \\
\hline PSB 7A & $\mathbf{U}$ & 0.2 & UGL & Mercury, total recoverable \\
\hline $\mathrm{R} \quad 6$ & $\mathrm{U}$ & 0.2 & UGL & Mercury, total recoverable \\
\hline RAC 2 & U & 0.2 & UGL & Mercury, total recoverable \\
\hline RAC 3 & $\mathrm{U}$ & 0.2 & UGL & Mercury, total recoverable \\
\hline RBW 1CL & $\mathrm{U}$ & 0.2 & UGL & Mercury, total recoverable \\
\hline $\mathrm{RBW} 2 \mathrm{CL}$ & $\mathrm{U}$ & 0.2 & UGL & Mercury, total recoverable \\
\hline $\mathrm{RCP} 1 \mathrm{~A}$ & $\mathrm{U}$ & 0.2 & UGL & Mercury, total recoverable \\
\hline RDB 1D & $\mathbf{U}$ & 0.2 & UGL & Mercury, total recoverable \\
\hline RDB 2D & $\mathrm{U}$ & 0.2 & UGL & Mercury, total recoverable \\
\hline RDB 3D & $\mathrm{U}$ & 0.2 & UGL & Mercury, total recoverable \\
\hline RRP 1 & $\mathrm{U}$ & 0.2 & UGL & Mercury, total recoverable \\
\hline RRP 2 & $\mathrm{U}$ & 0.2 & UGL & Mercury, total recoverable \\
\hline RRP 3 & $\mathrm{U}$ & 0.2 & UGL & Mercury, total recoverable \\
\hline RRP 4 & $\mathrm{U}$ & 0.2 & UGL & Mercury, total recoverable \\
\hline RSA 7 & $\mathrm{U}$ & 0.2 & $\mathrm{UGL}$ & Mercury, total recoverable \\
\hline RSA 8 & $\mathrm{U}$ & 0.2 & UGL & Mercury, total recoverable \\
\hline RSB 7 & $\mathrm{U}$ & 0.2 & UGL & Mercury, total recoverable \\
\hline $\mathrm{RSC} 10$ & $\mathrm{U}$ & 0.2 & UGL & Mercury, total recoverable \\
\hline RSD 1 & $\mathrm{U}$ & 0.2 & UGL & Mercury, total recoverable \\
\hline RSD 2A & $\mathrm{U}$ & 0.2 & UGL & Mercury, total recoverable \\
\hline RSD 2B & $\mathrm{U}$ & 0.2 & UGL & Mercury, total recoverable \\
\hline $\mathrm{RSD} 2 \mathrm{C}$ & $\mathrm{U}$ & 0.2 & UGL & Mercury, total recoverable \\
\hline RSD 3 & $\mathrm{U}$ & 0.2 & UGL & Mercury, total recoverable \\
\hline $\mathrm{RSD} 5$ & $\mathbf{U}$ & 0.2 & UGL & Mercury, total recoverable \\
\hline RSD 7 & $\mathrm{U}$ & 0.2 & UGL & Mercury, total recoverable \\
\hline RSD 9 & $\mathbf{U}$ & 0.2 & UGL & Mercury, total recoverable \\
\hline RSD 11 & $\mathrm{U}$ & 0.2 & UGL & Mercury, total recoverable \\
\hline RSE 1A & $\mathbf{U}$ & 0.2 & UGL & Mercury, total recoverable \\
\hline RSE 1B & $\mathrm{U}$ & 0.2 & UGL & Mercury, total recoverable \\
\hline RSE $1 \mathrm{C}$ & $\underline{U}$ & 0.2 & UGL & Mercury, total recoverable \\
\hline RSE 2 & $\mathbf{U}$ & 0.2 & UGL & Mercury, total recoverable \\
\hline RSE 3A & $\mathrm{U}$ & 0.2 & UGL & Mercury, total recoverable \\
\hline RSE 7 & $\mathrm{U}$ & 0.2 & UGL & Mercury, total recoverable \\
\hline
\end{tabular}


Wells $(\mathrm{n}=856$ ) with no estimated median mercury concentration (all values nondetect at or below nominal D.L.)

\begin{tabular}{|c|c|c|c|c|}
\hline well & qualifier & result & units & analyte_name \\
\hline RSE 8 & $\mathrm{U}$ & 0.2 & UGL & Mercury, total recoverable \\
\hline RSE 9 & $\overline{\mathrm{U}}$ & 0.2 & $\overline{\mathrm{UGL}}$ & Mercury, total recoverable \\
\hline RSE 10 & $\mathrm{U}$ & 0.2 & UGL & Mercury, total recoverable \\
\hline RSE 11 & $\mathrm{U}$ & 0.2 & UGL & Mercury, total recoverable \\
\hline RSE 13 & $\mathrm{U}$ & 0.2 & UGL & Mercury, total recoverable \\
\hline RSE 24 & $\mathrm{U}$ & 0.2 & UGL & Mercury, total recoverable \\
\hline RSE 25 & $\mathrm{U}$ & 0.2 & UGL & Mercury, total recoverable \\
\hline RSF 1 & $\mathbf{U}$ & 0.2 & UGL & Mercury, total recoverable \\
\hline RSF 2 & $\bar{U}$ & 0.2 & UGL & Mercury, total recoverable \\
\hline RSF 3 & $\mathrm{U}$ & 0.2 & UGL & Mercury, total recoverable \\
\hline RWM 4 & $\mathrm{U}$ & 0.2 & UGL & Mercury, total recoverable \\
\hline SBG 2 & $\mathrm{U}$ & 0.2 & UGL & Mercury, total recoverable \\
\hline SBG 3 & $\mathrm{U}$ & 0.2 & UGL & Mercury, total recoverable \\
\hline SBG 4 & $\mathrm{U}$ & 0.2 & UGL & Mercury, total recoverable \\
\hline SBG 5 & $\mathrm{U}$ & 0.2 & UGL & Mercury, total recoverable \\
\hline SBG 6 & $\mathrm{U}$ & 0.2 & UGL & Mercury, total recoverable \\
\hline SCA 1 & $\mathrm{U}$ & 0.2 & UGL & Mercury, total recoverable \\
\hline SCA 3A & $\mathrm{U}$ & 0.2 & UGL & Mercury, total recoverable \\
\hline SCA 4 & $\bar{U}$ & 0.2 & UGL & Mercury, total recoverable \\
\hline SCA 4A & $U$ & 0.2 & UGL & Mercury, total recoverable \\
\hline SCA 5 & $\mathrm{U}$ & 0.2 & UGL & Mercury, total recoverable \\
\hline$\overline{S C A} 6$ & $\bar{U}$ & 0.2 & UGL & Mercury, total recoverable \\
\hline SLP 1 & $\mathrm{U}$ & 0.2 & UGL & Mercury, total recoverable \\
\hline SLP 2 & $\overline{\mathrm{U}}$ & 0.2 & UGL & Mercury, total recoverable \\
\hline SRW 1BB & $\mathrm{U}$ & 0.2 & UGL & Mercury, total recoverable \\
\hline SRW 3A & $\mathrm{U}$ & 0.2 & UGL & Mercury, total recoverable \\
\hline SRW 3BB & $\mathrm{U}$ & 0.2 & UGL & Mercury, total recoverable \\
\hline SRW 7 & $\mathrm{U}$ & 0.2 & UGL & Mercury, total recoverable \\
\hline SRW 8 & $\mathrm{U}$ & 0.2 & UGL & Mercury, total recoverable \\
\hline SRW 8BB & $\mathrm{U}$ & 0.2 & UGL & Mercury, total recoverable \\
\hline SRW 9 & $\mathrm{U}$ & 0.2 & UGL & Mercury, total recoverable \\
\hline SRW 9A & $\mathrm{U}$ & 0.2 & UGL & Mercury, total recoverable \\
\hline SRW 9B & $\mathrm{U}$ & 0.2 & UGL & Mercury, total recoverable \\
\hline SRW 10BB & $\mathrm{U}$ & 0.2 & UGL & Mercury, total recoverable \\
\hline SRW 11 & $\mathbf{U}$ & 0.2 & UGL & Mercury, total recoverable \\
\hline SRW 11BB & $\mathrm{U}$ & 0.2 & UGL & Mercury, total recoverable \\
\hline SRW $12 \mathrm{~A}$ & $\mathrm{U}$ & 0.2 & UGL & Mercury, total recoverable \\
\hline SRW 12B & $\mathrm{U}$ & 0.2 & UGL & Mercury, total recoverable \\
\hline SRW 12C & $U$ & 0.2 & UGL & Mercury, total recoverable \\
\hline SRW 13A & $\mathbf{U}$ & 0.2 & UGL & Mercury, total recoverable \\
\hline SRW 13B & $\mathrm{U}$ & 0.2 & UGL & Mercury, total recoverable \\
\hline SRW 13C & $\bar{U}$ & 0.2 & UGL & Mercury, total recoverable \\
\hline SRW 14B & $\mathrm{U}$ & 0.2 & UGL & Mercury, total recoverable \\
\hline SRW 14C & $\mathrm{U}$ & 0.2 & UGL & Mercury, total recoverable \\
\hline SRW 15B & $\mathrm{U}$ & 0.2 & UGL & Mercury, total recoverable \\
\hline SRW $15 \mathrm{C}$ & $\mathrm{U}$ & 0.2 & $\mathrm{UGL}$ & Mercury, total recoverable \\
\hline SRW 16A & $\mathrm{U}$ & 0.2 & UGL & Mercury, total recoverable \\
\hline SRW 16C & U & 0.2 & UGL & Mercury, total recoverable \\
\hline SRW 17BB & $\mathrm{U}$ & 0.2 & UGL & Mercury, total recoverable \\
\hline SSS 2 & $\mathrm{U}$ & 0.2 & UGL & Mercury, total recoverable \\
\hline SSS 3 & $\mathbf{U}$ & 0.2 & UGL & Mercury, total recoverable \\
\hline SSS 4 & $\mathbf{U}$ & 0.2 & UGL & Mercury, total recoverable \\
\hline
\end{tabular}




\begin{tabular}{|c|c|c|c|c|}
\hline \multicolumn{5}{|c|}{$\begin{array}{l}\text { Wells ( } \mathrm{n}=856 \text { ) with no estimated median mercury concentration (all values nondetect at or } \\
\text { below nominal D.L.) }\end{array}$} \\
\hline well & qualifier & result & units & $\begin{array}{c}\text { analyte_name } \\
\end{array}$ \\
\hline SSS 19 & $\mathrm{U}$ & 0.2 & UGL & Mercury, total recoverable \\
\hline SSS 21 & $\mathrm{U}$ & 0.2 & UGL & Mercury, total recoverable \\
\hline SSS 26 & $\underline{U}$ & 0.2 & UGL & Mercury, total recoverable \\
\hline STR2220 & $\overline{\mathrm{U}}$ & 0.2 & UGL & Mercury, total recoverable \\
\hline TBG 5 & $\bar{U}$ & 0.2 & UGL & Mercury, total recoverable \\
\hline TNX 2D & $\mathrm{U}$ & 0.2 & UGL & Mercury, total recoverable \\
\hline TNX 7D & $\mathrm{U}$ & 0.2 & UGL & Mercury, total recoverable \\
\hline TNX 8D & $\mathrm{U}$ & 0.2 & UGL & Mercury, total recoverable \\
\hline TNX 9D & $\mathrm{U}$ & 0.1 & UGL & Mercury, total recoverable \\
\hline TNX 10D & $\mathrm{U}$ & 0.2 & UGL & Mercury, total recoverable \\
\hline TNX 12D & $\mathrm{U}$ & 0.2 & UGL & Mercury, total recoverable \\
\hline TNX 23D & $\mathrm{U}$ & 0.2 & UGL & Mercury, total recoverable \\
\hline TNX 24D & $\mathrm{U}$ & 0.2 & UGL & Mercury, total recoverable \\
\hline TNX 27D & $\mathrm{U}$ & 0.2 & UGL & Mercury, total recoverable \\
\hline YSC 1C & $\mathrm{U}$ & 0.2 & UGL & Mercury, total recoverable \\
\hline YSC 2A & $\mathrm{U}$ & 0.2 & UGL & Mercury, total recoverable \\
\hline YSC 2D & $\mathrm{U}$ & 0.2 & UGL & Mercury, total recoverable \\
\hline YSC $4 \mathrm{C}$ & $\mathrm{U}$ & 0.2 & UGL & Mercury, total recoverable \\
\hline YSC 5A & $\mathrm{U}$ & 0.2 & UGL & Mercury, total recoverable \\
\hline ZBG 1A & $\mathrm{U}$ & 0.2 & UGL & Mercury, total recoverable \\
\hline ZDT 1 & $\mathrm{U}$ & 0.2 & UGL & Mercury, total recoverable \\
\hline ZDT 2 & $\mathrm{U}$ & 0.2 & UGL & Mercury, total recoverable \\
\hline$Z W \quad 1 \mathrm{~A}$ & $\mathrm{U}$ & 0.2 & UGL & Mercury, total recoverable \\
\hline $\mathrm{ZW} 8$ & $\mathbf{U}$ & 0.2 & UGL & Mercury, total recoverable \\
\hline
\end{tabular}

